\title{
The Plasma and Suprathermal Ion Composition (PLASTIC) Investigation on the STEREO Observatories
}

\author{
A.B. Galvin - L.M. Kistler • M.A. Popecki · C.J. Farrugia - K.D.C. Simunac • L. Ellis • \\ E. Möbius · M.A. Lee · M. Boehm · J. Carroll • A. Crawshaw • M. Conti • P. Demaine · \\ S. Ellis · J.A. Gaidos • J. Googins • M. Granoff • A. Gustafson • D. Heirtzler • B. King • \\ U. Knauss · J. Levasseur $\cdot$ S. Longworth $\cdot$ K. Singer $\cdot$ S. Turco $\cdot$ P. Vachon • \\ M. Vosbury • M. Widholm • L.M. Blush • R. Karrer • P. Bochsler • H. Daoudi • \\ A. Etter · J. Fischer · J. Jost • A. Opitz • M. Sigrist • P. Wurz • B. Klecker • M. Ertl • \\ E. Seidenschwang $\cdot$ R.F. Wimmer-Schweingruber $\cdot$ M. Koeten $\cdot$ B. Thompson • \\ D. Steinfeld
}

Received: 9 February 2007 / Accepted: 30 November 2007 / Published online: 29 January 2008 (C) Springer Science+Business Media B.V. 2008

\begin{abstract}
The Plasma and Suprathermal Ion Composition (PLASTIC) investigation provides the in situ solar wind and low energy heliospheric ion measurements for the NASA Solar Terrestrial Relations Observatory Mission, which consists of two spacecraft (STEREOA, STEREO-B). PLASTIC-A and PLASTIC-B are identical. Each PLASTIC is a timeof-flight/energy mass spectrometer designed to determine the elemental composition, ionic
\end{abstract}

\footnotetext{
A.B. Galvin $(\varangle) \cdot$ L.M. Kistler · M.A. Popecki · C.J. Farrugia · K.D.C. Simunac · L. Ellis · E. Möbius · M.A. Lee · M. Boehm · J. Carroll · A. Crawshaw · M. Conti · P. Demaine - S. Ellis · J.A. Gaidos · J. Googins · M. Granoff · A. Gustafson · D. Heirtzler · B. King · U. Knauss · J. Levasseur · S. Longworth $\cdot$ K. Singer $\cdot$ S. Turco $\cdot$ P. Vachon $\cdot$ M. Vosbury $\cdot$ M. Widholm Institute for the Study of Earth, Oceans and Space, University of New Hampshire, SSC Morse Hall, Durham, NH 03824, USA

e-mail: toni.galvin@unh.edu
}

A.B. Galvin · L.M. Kistler · C.J. Farrugia · K.D.C. Simunac · E. Möbius · M.A. Lee Department of Physics, University of New Hampshire, SSC Morse Hall, Durham, NH 03824, USA

L.M. Blush · R. Karrer · P. Bochsler · H. Daoudi · A. Etter · J. Fischer · J. Jost · A. Opitz · M. Sigrist · P. Wurz

Physikalisches Institut, University of Bern, Sidlerstrasse 5, 3012 Bern, Switzerland

B. Klecker · M. Ertl · E. Seidenschwang

Max-Planck-Institut fuer extraterrestrische Physik, Postfach 1312, 85741 Garching, Germany

R.F. Wimmer-Schweingruber · M. Koeten

Extraterrestrial Physics, Institute for Experimental and Applied Physics, Christian-Albrechts-University Kiel, Leibnizstr. 11, 24098 Kiel, Germany

B. Thompson

National Aeronautics and Space Administration, Goddard Space Flight Center, Greenbelt, MD 20771, USA

D. Steinfeld

Orbital Sciences Corporation, 7500 Greenway Center Drive, Suite 700, Greenbelt, MD 20770, USA 
charge states, and bulk flow parameters of major solar wind ions in the mass range from hydrogen to iron. PLASTIC has nearly complete angular coverage in the ecliptic plane and an energy range from $\sim 0.3$ to $80 \mathrm{keV} / \mathrm{e}$, from which the distribution functions of suprathermal ions, including those ions created in pick-up and local shock acceleration processes, are also provided.

Keywords STEREO - Solar wind · Plasma · Suprathermal · Composition · Time-of-flight spectrometer $\cdot$ Coronal mass ejections $\cdot$ Multipoint spacecraft observations

\section{Introduction}

The Solar TErrestrial RElations Observatory (STEREO) is the third strategic mission in NASA's Solar Terrestrial Probes (STP) Program and consists of two spacecraft that are drifting apart in solar longitude by $45^{\circ}$ per year. The primary science goal for the STEREO mission is to understand the origin and consequences of Coronal Mass Ejections (CMEs), including their interplanetary manifestations (ICMEs). The specific scientific objectives (Kaiser et al. 2007) are to:

- Understand the causes and mechanisms of CME initiation,

- Characterize the propagation of ICMEs through the inner heliosphere to $1 \mathrm{AU}$,

- Discover the mechanisms and sites of solar energetic particle (SEP) acceleration, in the low corona and in the interplanetary medium, and

- Develop a three-dimensional, time-dependent model of the magnetic topology and plasma temperature, density and velocity structure of the ambient solar wind.

As a secondary objective, STEREO will

- Provide a continuous, low rate data stream ("Beacon Mode") for the purposes of space weather specification and possible prediction of geomagnetic storms.

A science payload consisting of four investigations is on each observatory. Two remote imaging investigations (SECCHI, SWAVES) are used for continuous viewing of solar features and to track transients such as CMEs and shocks traveling toward Earth's orbit. Two in situ investigations (IMPACT, PLASTIC) continuously sample the particles and magnetic fields of associated heliospheric structures and phenomena (including CIRs, ICMEs and SEPs) as they pass by the spacecraft near 1 AU. The SECCHI instrument suite is described by Howard et al. (2007), the SWAVES instrument by Bougeret et al. (2007), and the IMPACT instrument suite by Luhmann et al. (2007). The PLASTIC instrument is described herein. These experiments, combined with the unique vantage points afforded by the STEREO spacecraft drifting orbits, are designed to meet the mission science objectives.

The STEREO mission timeline can be roughly divided into two operational stages, ordered by their orbits and payload status. The "phasing orbit stage" took place during the first three months after launch (late October 2006 through January 2007). The leading (in heliocentric orbit) spacecraft is denoted as "STEREO-A" (AHEAD) and the lagging spacecraft as "STEREO-B" (BEHIND). The observatories, while in the phasing stage, were in geocentric orbits that included one (A) or two (B) gravity-assist lunar swing-bys. Most spacecraft and instrument commissioning activities took place during this near-Earth stage. Thereafter began the primary data or "science orbit stage" where both observatories had attained their respective heliocentric orbits, drawing away from each other and from the Earth. The spacecraft are currently at $\sim 1 \mathrm{AU}$, drifting away from the Earth-Sun line at a rate of $\sim 22.5^{\circ}$ per 
year. Using these drifting orbits, the two observatories combine measurements in the ecliptic plane from two different vantage points in solar longitude. This unique orbit configuration allows the STEREO spacecraft to image the Sun in a three dimensional perspective.

The Plasma and Suprathermal Ion Composition (PLASTIC) investigation team on STEREO includes participants from the University of New Hampshire (USA), the University of Bern (Switzerland), the Max-Planck-Institute for extraterrestrial Physics (Germany), the Christian-Albrechts-University Kiel (Germany), and NASA Goddard Space Flight Center (USA). Instrument Data Processing Unit (IDPU) functions for PLASTIC, as well as the PLASTIC LVC and its EM Interference Filter, were provided by the IMPACT investigation (Luhmann et al. 2007). Specific contributions from these institutions are further summarized in the acknowledgements.

\section{STEREO PLASTIC Scientific Objectives}

The Plasma and Suprathermal Ion Composition (PLASTIC) instrument on STEREO is designed to study in situ the bulk properties of solar wind (SW) protons and the composition and properties of solar wind minor ions. PLASTIC also measures the composition, spectra, and anisotropy of heliospheric suprathermal (ST) ions in the energy-per-charge range from $\sim 0.3$ to $80 \mathrm{keV} / \mathrm{e}$. (See Table 1 for additional details.) With these measurements, PLASTIC is the primary sensor on STEREO for studying the link between coronal and solar wind ion processes, and between solar wind and heliospheric processes, as discussed below.

\subsection{Solar Processes and Solar Wind Studies}

The elemental and isotopic composition of the solar wind observed at $1 \mathrm{AU}$ is determined in the low solar atmosphere and in the corona. Deviations from photospheric abundances indicate that various fractionation processes are involved in the solar material that is fed into the corona and in the subsequent extraction of some portion of this material into the solar wind (Wimmer-Schweingruber 2002, and references therein). The solar wind composition provides in essence a "DNA sample" of its origin, which survives even situations where the solar wind kinetic properties have been dramatically altered, such in corotating or stream interaction regions.

Charge state distributions of minor species in the solar wind can be related (via models) to coronal electron temperature-, electron density-, and ion velocity-profiles obtained from optical observations along the solar wind flow tube and particularly from the region where the charge states effectively freeze-in (e.g., Aellig et al. 1997; Ko et al. 1998). Such observations are used, for example, to assess the role of suprathermal electrons and electron anisotropies in the freezing-in process (Ko et al. 1996). Solar wind compositional signatures can also be used to infer small-scale changes (on the order of the supergranulation) in the corona. Thus, composition measurements give detailed knowledge of the coronal structure in the formation region of the solar wind.

Compositional signatures, such as abundance variations related to the first ionization potential (FIP) or first ionization time (FIT) effects, seem to be temporally and spatially related to the charge-state composition of minor species and to dynamic properties of the ambient solar wind flow. The details of this link are still not understood. The processes affecting coronal/solar wind elemental abundances and charge state distributions operate at very different sites between the solar surface and $1 \mathrm{AU}$, yet they seem to be closely correlated. Observing optically the conditions off the solar limb and comparing them to in 
situ measurements of particles and magnetic fields will provide useful clues on the nature of such correlations. It is especially advantageous to carry out these studies over a series of transitions from slow to fast or fast to slow solar wind during the early phase of the STEREO mission (which coincides with solar activity minimum).

The origin of the slow solar wind is still a controversial issue. The in-depth case study presented by Ko et al. (2006) illustrates both the importance and the complexity of relating in situ solar wind elemental and charge state measurements, models, and solar limb spectroscopic observations to resolve this issue. Limb measurements of solar features (such as streamers, active regions, and coronal holes) that are potential sources of the solar wind are taken about seven days before or after that feature passes central meridian. Since the solar wind travels in a radial direction outward from the Sun, in-situ composition measurements are restricted to solar wind originating from near the disk's center (from the perspective of the spacecraft). This leads to a significant temporal offset in the corresponding optical and in-situ measurements. There have been a limited number of quadrature measurements between Ulysses and near-Earth solar observatories (e.g. Poletto et al. 2001). Ulysses carries a sophisticated solar wind composition spectrometer that has provided major new solar wind discoveries (Gloeckler et al. 1992). However, Ulysses limb passages are restricted to two per year and correlations can be affected by uncertainties in estimating the solar originating longitudes, introduced by the long solar wind transit distances to the Ulysses orbit. Solar wind and optical correlations are further hampered by optical ambiguities, notably line-of-sight affects. The twin STEREO spacecraft will advance our understanding of the sources of the solar wind by removing some of these ambiguities. For the first time it is possible to relate variations in the solar wind properties, including composition, with the relevant temporal variations of features observed in the chromosphere and the corona.

The importance of such correlations is further illustrated, for example, by the observation of $\mathrm{O}^{+5}$ in situ (Wimmer-Schweingruber et al. 1998). A SOHO/UVCS observation of this ion in the corona implies that the $\mathrm{O}^{+5}$ outflow velocity enhancement relative to protons must decrease with increasing radius (Cranmer et al. 1999). With STEREO, velocity profiles and velocity distributions of species obtained in the inner corona by means of the Doppler dimming technique can be matched with the corresponding velocity distributions of such species in situ-thus allowing a better match of all types of solar wind and coronal conditions than previously achieved.

STEREO PLASTIC measures all major solar wind ion species under all conditions. It has an exceptional directional geometrical factor (active area) for ions of nuclear charge state $Z>2$, and it determines the flow direction for selected $Z>2$ ions. With STEREO, we will be able to draw substantial new conclusions for the slow and fast solar wind as well as for transient phenomena.

\subsection{Interplanetary Manifestations of Coronal Mass Ejections (ICME)}

One of the anticipated achievements of STEREO will be the ability to remotely observe the launch of a CME and the propagation of it and its shock from the Sun out to $1 \mathrm{AU}$ with the remote imagers (SECCHI and SWAVES) from one STEREO observatory, and then subsequently measuring its interplanetary properties with the in situ instruments (IMPACT and PLASTIC) as the ICME passes the other STEREO observatory. In combination with modelling and theory (Aschwanden et al. 2007; Forbes et al. 2006), this unique configuration will allow us to causally relate the solar and interplanetary phenomena.

Although much is known about CMEs and ICMEs, many fundamental questions remain regarding their origin, evolution and propagation. The reader is referred to the AGU monograph (Geophysical Monograph 99: Coronal Mass Ejections, 1997) and the more recent 
Space Science Reviews special issue (March 2006) for extensive reviews on our current state of knowledge on ICMEs. Some of the outstanding questions have been summarized by Wimmer-Schweingruber (2006). The reader is also referred to Luhmann et al. (2007). Here we highlight a few of the open questions that STEREO will strive to answer.

\subsubsection{CME Structure on the Sun and Related In Situ Signatures}

A "three-part structure" is often observed in white light coronagraph images of eruptive prominence-associated CMEs: a leading bright shell, followed by a dark region, and finally a dense bright core. These are believed to correspond to, respectively, the pre-eruption dome of the helmet streamer that overlies the prominence, the cavity surrounding the prominence in its quiet state, and the embedded quiescent prominence (e.g., Hundhausen 1988; Low 1994). The material blown into space would thus originate from the low corona or chromosphere. If ICMEs involve and retain closed magnetic topologies, this will be reflected in the magnetic field and electron structures observed in situ by IMPACT. Bulk properties, composition, and ionization state of the solar wind ions will be measured by PLASTIC.

ICMEs exhibit a tremendous variability in their internal structure and composition, as described by Galvin (1997) and illustrated in the study of 41 ICMEs by Neukomm (1998). Elevated charge states are often observed (e.g., Galvin et al. 1987, 1993; Galvin 1997; Lepri et al. 2001; Lepri and Zurbuchen 2004; Richardson and Cane 2004a), as well as unusual elemental composition (e.g., Richardson and Cane 2004a). As discussed by WimmerSchweingruber et al. (2006), the frozen-in ionic charge states of the solar wind plasma appear to provide a direct link to the CME initiation process on the Sun, while the elemental composition provides information on the solar atmosphere prior to the CME initiation. With the quadrature measurements of STEREO, remote imaging of the Sun (both prior to and during the CME initiation) can be linked with the in situ sampling of the associated ICME.

There have been some reported cases of ICME-related solar wind containing singly charged $\mathrm{He}$, with observed $\mathrm{He}^{+} / \mathrm{He}^{+2}$ ranging from less than $1 \%$ to as much as $30 \%$ (see review by Bame 1983), and other low charge state observations for minor ions (e.g., Gloeckler et al. 1999). Are these low charge states and unusual composition a tag for chromospheric material? Or are they rather associated with a reconnection process at the Sun, as suggested by Riley et al. (2002)? The combined observations by the remote imagers and the in situ investigations may provide the opportunity to delineate the origin of these unusual compositional features.

\subsubsection{ICME Boundaries}

As described, for example, by Galvin (1997) and more extensively by Zurbuchen and Richardson (2006), there are several in situ signatures used to identify ICMEs in interplanetary space; however, not all signatures are normally seen for a given event, and some signatures are not even unique to ICMEs (see also Neugebauer and Goldstein 1997). In addition, the boundaries associated with ICMEs can be ambiguous, and different boundaries are often observed for different signatures (see case study by Galvin et al. 1987, and discussion by Wimmer-Schweingruber et al. 2006). Multiple magnetic field discontinuities (some supporting waves), magnetic holes, and other substructures may be involved (Crooker et al. 1990; Farrugia et al. 2001; Janoo et al. 1998; Vasquez et al. 2001). Multi-spacecraft observations of a given ICME provide spatial and temporal tracking of these boundaries, and help to distinguish cases of merged (coalescing) ICMEs. This type of study requires a full constellation of spacecraft, such as ACE, Wind, STEREO, SOHO and Ulysses. 


\subsubsection{The Evolution of ICMEs in the Inner Heliosphere}

One of the scientific objectives of STEREO is to follow the evolution of ICMEs from the Sun to $1 \mathrm{AU}$. Of perhaps special interest in the early phase of the mission is the subset of ICMEs known as magnetic clouds (MCs). ICMEs occurring near solar minimum have been statistically associated with MCs (Richardson and Cane 2004b), and in all stages of the solar cycle they form a substantial fraction of observed ICMEs.

Magnetic clouds (MC) are defined as a mesoscale structure characterized by a strong magnetic field executing a large rotation in plasma of low proton beta (Burlaga et al. 1981). They have been modelled as cylindrically symmetric, force-free configurations; i.e., as solutions of $\nabla \times \underline{\mathbf{B}}=\alpha(\underline{\mathbf{r}}) \underline{\mathbf{B}}$ (Goldstein 1983; Marubashi 1986). The case for $\alpha(\underline{\mathbf{r}})$ equals a constant was proposed by Burlaga (1988), and the magnetic filed components can be written in terms of Bessel functions of zeroth and first order (Lundquist 1950). Fitting of in situ data as a force-free magnetic flux tube has become a standard approach to provide a cylindrical geometry solution for the cloud. From this solution, one may infer the magnetic helicity (or handedness) of the field, the orientation of the axis, the maximum field on the axis, and the closest distance the spacecraft passes from the axis. More recent approaches include reconstruction of the magnetic field, flux tube orientation, etc., based on the GradShafranov equation (Hu and Sonnerup 2002), and using kinematic expansions of the clouds (Riley and Crooker 2004). These recent modelling efforts suggest deviations from a circular cross-section.

When in situ data are available from a constellation of spacecraft at different locations, these modelled reconstructions can be used as a "probe" on the cloud's internal structure, with a direct comparison to spatial and temporal variations in plasma properties observed within the cloud (Farrugia et al. 2005a; Leitner et al. 2007). This is an ideal situation for the STEREO, ACE, and Wind constellation. The wide spacecraft separation may allow an investigation of the spatial structure and coherence lengths of ICME parameters over longitudinal separations of ten's of degrees. Earlier studies (Farrugia et al. 2005b, 2005c) have provided cross-correlating parameters of magnetic clouds using the largest separations (a few $100 \mathrm{Re}$ ) attained between the Wind and ACE spacecraft—both along, and orthogonal to, the bulk solar wind flow vector. These studies may now be extended to larger spatial scales.

\subsection{Injection and Acceleration of Ions at CME-Driven Shocks}

With the PLASTIC and IMPACT investigations, each STEREO covers an extensive particle energy range ( $\sim$ few eV to $\sim 100 \mathrm{MeV})$. The combined composition measurements, in particular the ability of PLASTIC to measure charge states and distinguish pickup ions, are ideally suited to study the 3-dimensional evolution of suprathermal and energetic particles into the heliosphere, including the injection, acceleration, and elemental fractionation processes. The suprathermal range of PLASTIC (up to $80 \mathrm{keV} / \mathrm{e}$ ) covers injection and the standard power law of stationary planar shock acceleration, while the energy range of IMPACT SEP (up to $100 \mathrm{MeV}$ ) covers the signatures of ion escape from the shock, which in turn provide information on shock structure near the Sun.

Generally two classes of solar energetic particle (SEP) events are distinguished (Reames 1999). Impulsive events are typically characterized by low intensity, narrow angular spread, short duration, and enrichment in ${ }^{3} \mathrm{He}$, heavy ions, and electrons. These events are associated with solar flares and type III radio bursts. Gradual events are higher intensity, long-lived, proton rich, and show a broad angular spread. This class is associated with fast CMEs and type II radio bursts. 
CME/ICMEs are the most likely origin of large "gradual" SEP events that occur $\sim 10$ times per year during solar maximum. The correlation between fast CMEs and these SEPs is over 95\% (Kahler et al. 1984). Fast ICMEs drive shock waves into the solar wind, which strengthen as the Alfvén speed decreases with increasing heliocentric radial distance. The resulting shock wave can accelerate solar wind ions, pickup ions, or SEPs from previous gradual or impulsive events, up to energies occasionally in excess of $1 \mathrm{GeV} / \mathrm{n}$. A fraction of the higher energy ions continually escape the shock and propagate to the orbit of Earth, where their composition and energy spectra provide information on the conditions at the ICME and shock when they are closer to the Sun (for a recent summary on the characteristics of SEPs related to CMEs and ICMEs see Klecker et al. 2006). SEPs and the associated shock waves have important effects on Earth's space environment, and SEPs can pose radiation hazards for astronauts.

This two-class picture of impulsive and gradual events is oversimplified, as evident from recent results from instruments with improved collecting power and resolution onboard the Wind, SAMPEX, SOHO and ACE spacecraft (for a recent summary see von Rosenvinge and Cane 2006, and references therein). The new composition and ionic charge measurements indicate that some solar particles have their origin in a dense plasma low in the corona, even in events otherwise classified as "gradual" (Möbius et al. 1999; Popecki 2006, and references therein); that enrichments in ${ }^{3} \mathrm{He}$ are also common in interplanetary shock accelerated populations (Desai et al. 2001); and that enrichments in heavy ions are often observed in large events at high energies (Cane et al. 2003). It is heavily debated whether these new findings are best explained by a suprathermal seed population leftover from previous "impulsive" events (Mason et al. 1999); by the interplay of shock geometry and different seed populations such as solar wind and flare suprathermals (Tylka et al. 2005); or by direct injection from the flare acceleration process (e.g. Klein and Trottet 2001, and references therein; Cane et al. 2003) with or without further acceleration by a coronal shock.

Simulations indicate that locally accelerated solar wind protons and alphas are a likely source population for suprathermal ion distribution functions associated with interplanetary shock events observed by Ulysses (Baring et al. 1997). Indeed, both solar wind and pickup ions accelerated by various types of shocks (interplanetary and bow shocks) appear to contribute to the suprathermal populations as observed in the outer heliosphere by Ulysses (Gloeckler et al. 2005a). A crucial test for the various SEP acceleration models will consist of a detailed comparison of the suprathermal seed population with the characteristics of the more energetic particles. With its high time resolution and ability to observe ions both before and after injection, PLASTIC will be able to determine ion injection rates when the shock passes the two spacecraft, as functions of the magnetic field orientation and shock strength at each spacecraft. The temporal resolution of the ions and magnetic field fluctuations in the turbulent sheath will provide a good test of wave-particle interactions taking place in this region.

Recent observations from ACE have provided evidence of simultaneous changes in the Fe charge states in the solar wind and SEP populations during an ICME event (Popecki et al. 2000; Farrugia et al. 2002). Because the ICME structure in this event was connected to an active region on the Sun with ongoing activity, it has been difficult to determine (from the one observational point afforded by ACE) as to whether the high charge component of the energetic ions is accelerated out of the solar wind, or stems from an impulsive event. PLASTIC will extend such measurements down to the suprathermal region (just above solar wind energies). The two STEREO spacecraft will make it possible to distinguish between these two possible sources. 


\subsection{Heliospheric Studies}

Among the primary science objectives for STEREO is an enhanced understanding of the origins, properties, and evolution of heliospheric structures and phenomena. Phenomena of interest are not just magnetic clouds and other ICME-related structures, but also include stream interfaces, sector boundaries, co-rotating interaction regions (CIR events), and pickup ions.

\subsubsection{Stream Interaction Regions and Heliospheric Current Sheet}

During the last solar minimum in 1996, the Whole Sun Month campaign (Galvin and Kohl 1999) provided an unprecedented opportunity for observers and modelers to connect the global corona to structures seen in the solar wind (Gibson 2001). STEREO has been launched near the minimum activity phase of the current solar cycle and will contribute to a new, enhanced global perspective of the solar minimum heliosphere.

The large-scale corona at this time is mostly defined by long-lived coronal holes (including the polar holes) and the equatorial streamer belt. These coronal structures are reflected within the global heliosphere as a relatively stable pattern of high-speed streams and interstream ("slow") solar wind that interact dynamically as the Sun rotates. The evolution of these large-scale structures, known as stream interaction regions or CIRs, is of primary interest to both heliospheric studies and space weather prediction. A recent review by Riley (2007) gives an overview of the current state of observations and models of the evolution of CIRs from the Sun to 1 AU. The two sets of STEREO in situ measurements, combined with those at the Earth solar longitude, allow for the first time a determination of the temporal evolution of these corotating structures and an investigation of their structure in the longitudinal direction (i.e., along the Earth's orbital direction). Any changes can then be directly compared with the solar observations of temporal changes in the corresponding solar wind source longitude.

Stream interfaces mark the boundary of high-speed streams and the slow, interstream, solar wind. Stream interfaces are often embedded in the leading edge of CIRs. Identification of the stream interface is important for many heliospheric studies, including the study of recurrent particle events and current sheet dynamics. While stream interfaces are often identified by a change in entropy, this signal can, in some cases, be misleading. The safest identification includes the compositional signatures of the solar wind (Wimmer-Schweingruber et al. 1997, 1999).

\subsubsection{Particle Acceleration at Corotating Interaction Regions}

The launch of STEREO during the approach to solar minimum provides an opportunity to study the recurrent ion events associated with corotating interaction regions (CIRs). These CIR-related events are known to be an important source of the low-energy interplanetary particle populations during periods of low solar activity (Reames 1999, and references therein). CIRs continue to develop beyond the orbit of the Earth; and, indeed, it is typically beyond $1 \mathrm{AU}$ that a forward and reverse shock pair forms. Observations with many spacecraft have shown that the strongest particle acceleration occurs at the reverse shock (e.g. review by Mason et al. 1999); that there is strong cross-field transport in intense events (Dwyer et al. 1997); and that the contribution of singly charged ions (C, O, Ne, $\mathrm{Mg}$, or Fe) to the source population of CIRs is at most a few per cent (Mazur et al. 2002; Möbius et al. 2002). 
These observations suggest the solar wind as a possible source; however, compositional peculiarities observed for $\mathrm{He}, \mathrm{C}$, and $\mathrm{Ne}$ in CIRs are not entirely consistent with this scenario, or at least suggest that more than one seed population is involved. Pickup $\mathrm{He}^{+}$has been observed as accelerated suprathermals at a CIR reverse shock at $>2 \mathrm{AU}$ (Gloeckler et al. 2005b), and in CIR compression regions at $1 \mathrm{AU}$ (Chotoo et al. 2000). The C/O-ratio has been found to be different from both solar wind and solar energetic particle abundances, and to vary systematically with solar wind speed (Mason et al. 1997). A small, but noticeable $\mathrm{Ne}^{+}$contribution has been measured, indicating that efficient pickup ion acceleration of heavies can occur, even if it is not a dominant source population at $1 \mathrm{AU}$ (Möbius et al. 2002). STEREO, by providing complete compositional and spectral information extending from solar wind and suprathermal energies (PLASTIC) to energies of several MeV/amu (IMPACT), is ideally suited for the in-situ study of these events and their source populations. Furthermore, by making use of multi-spacecraft measurements, a detailed analysis of spatial and temporal variations and anisotropies will be possible.

\subsubsection{Pickup Ions}

As seen in the "first light" section at the end of this paper, the compositional, angular, energy and flux coverage of PLASTIC is well-suited for the study of pickup ions, whether of interstellar or local origin. Pickup ions are of interest in their own right, and as a potential seed population.

Pickup ions originate from neutral atoms that become freshly ionized due to various mechanisms, and are "picked up" by the interplanetary magnetic field (e.g., Thomas 1978; Möbius et al. 1985; Gloeckler and Geiss 1998). Because the pickup process generates speeds up to twice the solar wind speed (in the spacecraft frame), these ions can be easily injected into acceleration mechanisms. As indicated earlier, pickup ions have been observed as a source population for particle acceleration at shocks and perhaps compression regions (Gloeckler et al. 2005b; Chotoo et al. 2000; Kucharek et al. 2003).

Studies of pickup ions have shown that their fluxes are controlled not just by ionization rates, but also by transport effects that vary with the interplanetary magnetic field and solar wind conditions (e.g., Möbius et al. 1998; Saul et al. 2007). Another observable effect on the flux of the interstellar pickup ions is the gravitational focusing cone that is formed on the downwind side of the Sun, seen from the Earth's perspective each December. Untangling spatial from temporal variations requires a three-dimensional constellation of heliospheric spacecraft. STEREO provides two additional simultaneous in-ecliptic measurements separated in solar longitude. These will be combined with near-Earth in-ecliptic observations (for example, by ACE and the future IBEX) and out-of-ecliptic measurements (Ulysses).

\subsection{Space Weather}

Part of the STEREO mission includes the real-time, continuous transmission of a limited number of space weather related products, called Beacon Mode (see Biesecker et al. 2007).

STEREO provides a test of the ability of in situ experiments at solar longitudes different than that of the Earth to issue real-time space weather warnings. In situ data from satellites along the Sun-Earth line at L1 are in a better position to issue warnings that an interplanetary CME is in a direct path to impact the Earth. However, the off-angle, $1 \mathrm{AU}$ orbits of the STEREO observatories provide a complementary aspect to space weather forecasting, particularly for recurrent high-speed streams, and the associated CIRs, prevalent during solar minimum conditions. They also provide a warning for transient flows that last for several days, but not for multiple solar rotations (Burlaga 1995). 
CIR-related storms have been associated with elevated spacecraft potentials, with subsequent effects on instrument performance (Denton et al. 2006). Using the lagging STEREO B spacecraft in situ measurements, STEREO is able to provide considerable advance notice and much more accurate estimates of the time and possible geo-effectiveness of these structures than can currently be issued by using only L1 satellites. (For example, an EarthSun-STEREO B angle of $20^{\circ}$ corresponds to 36 hours of solar rotation and additional advance notice.) With the STEREO spacecraft slowly drifting apart from Earth, STEREO will allow us to estimate the optimal spacecraft longitude for this type of forecast in planning future operational missions. In addition, CMEs launched while corotating streams are present have a tendency to be caught in the structure, thus affecting the CME propagation through space. In this context, knowledge of high-speed stream structures obtained by both STEREO spacecraft provides important information for space weather forecasting.

\section{Instrument Description}

\subsection{Overview}

The PLASTIC sensor (Fig. 1) is comprised of three structural elements: Entrance System (Energy/charge Analyzer), Time-of-Flight/Energy (TOF/E) Chamber and its Housing, and the Electronics Box (EBox). The Entrance System selects incident particles for subsequent analysis by their incoming direction and by their energy-per-charge $(E / Q)$ value. The Timeof-Flight/Energy Chamber contains the ion optics, detectors, detector electronics, and the signal processing board. The Electronics Box contains all of the digital electronics, the remaining analog electronics, the low and high voltage power supplies, and the connections to spacecraft power and to the IMPACT/PLASTIC Instrument Data Processing Unit (IDPU).

To accommodate measurements of both plasma and suprathermal populations within a single instrument package on a 3-axis stabilized platform, the PLASTIC sensor incorporates three distinct fields of view (FOV) and geometrical factors (GF). Each FOV/GF is combined with an instrument ion optics section optimized for a particular type of ion population. A summary of the PLASTIC instrument sections is given below, and their basic measurements are provided in Table 1.

The PLASTIC Solar Wind Sector (SWS) provides a $45^{\circ}$ field of view in the azimuth (nominally ecliptic) plane, centered on the Sun-spacecraft line, and $\pm 20^{\circ}$ in elevation (nominally polar) direction through the use of electrostatic deflectors. The SWS has two channels with different geometrical factors suited to the high flux solar wind protons and the low intensity solar wind minor ions, respectively.

- The Solar Wind Sector (SWS) Small Channel measures the distribution functions of solar wind protons $\left(\mathrm{H}^{+}\right)$and alphas $\left(\mathrm{He}^{+2}\right)$, providing proton density $(n)$, velocity $\left(V_{s w}\right)$, thermal speed $\left(v \_t h\right)$, and alpha to proton $\left(\mathrm{He}^{+2} / \mathrm{H}^{+}\right)$ratios.

- The Solar Wind Sector (SWS) Main Channel measures the elemental composition, ionic charge state distribution, and bulk and thermal speeds of the more abundant solar wind minor ions (e.g., C, O, Mg, $\mathrm{Si}$, and Fe).

The Suprathermal Ions Wide-Angle Partition Sector (WAP) covers $210^{\circ}$ (B) and $225^{\circ}$ (A) fields of view in the remaining unobstructed off-Sun azimuth directions, and $<10^{\circ}$ in the polar (centered on the ecliptic plane) direction. The WAP has a larger geometrical factor but less directional information than either of the two SWS channels.

The SWS and WAP share a common $360^{\circ}$ toroidal top-hat electrostatic analyzer with its azimuth entrance in the ecliptic plane. At the detector level, this full circular geometry is 
Fig. 1 Photograph of PLASTIC Flight Model, showing the three structural elements comprising the instrument: the Entrance System (domelike, gold color, with the Solar Wind Sector deflectors facing upwards), the Time-of-Flight/Energy Chamber and its Housing (cylindrical center section), and the Electronics Box. Cables running from beneath the SWS of the Entrance System down to the side of the EBox are the connections to the various high voltage power supplies for the Entrance System. Red and white cabling on the top of the EBox are attached to survival heaters

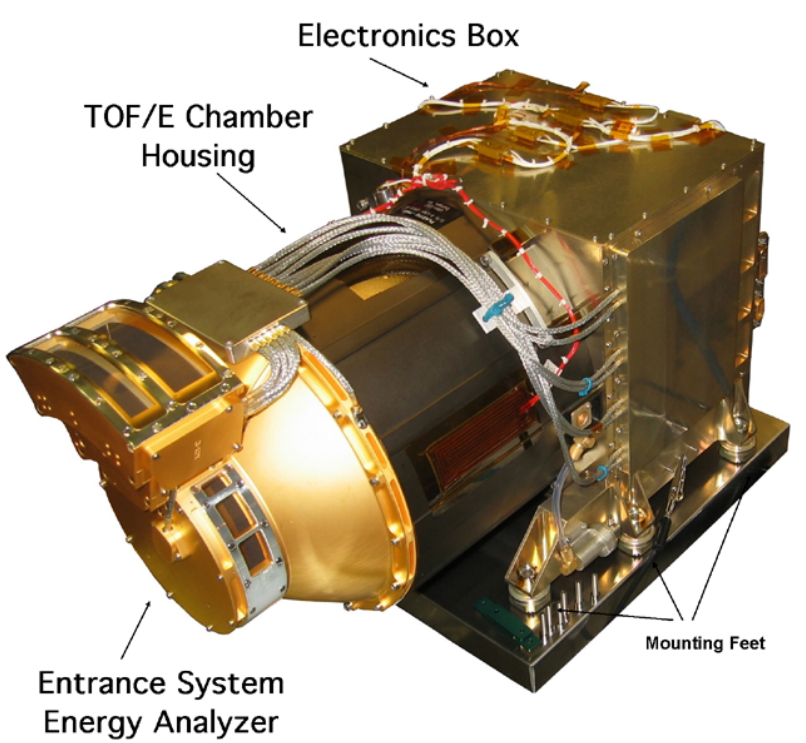

subdivided into four $90^{\circ}$ quadrants (designated Q0, Q1, Q2, and Q3, see insert of Fig. 2). Each quadrant has its own $90^{\circ}$ annular Micro-channel Plate (MCP). Different geometrical factors, viewing angles, measurement techniques, event logic conditions, and data products are used depending upon which sector and quadrant the incident particle traverses.

\subsection{Principles of Operation}

The PLASTIC sensor combines a top-hat $360^{\circ}$ electrostatic (energy-per-charge) analyzer (ESA) with instantaneous full aperture (in-ecliptic) coverage, followed by post-acceleration (PAC), a time-of-flight (TOF) system, and an energy $(E)$ measurement by solid state detectors (SSD) in selected portions (Fig. 2). The detector area is divided into four 90-degree quadrants (Q0-Q3). The Entrance System is divided into a $45^{\circ}$ sunward-centered solar wind sector (SWS) with polar deflectors and the remaining wide-angle partition (WAP) sector without polar deflectors. There are three geometrical factors employed in the Entrance System. The electrostatic deflectors enable solar wind angular coverage outside of the ESA aperture plane, up to $20^{\circ}$ elevation above and below the ecliptic plane for the three-axisstabilized STEREO spacecraft. Discrete anodes (coarse angular resolution, 22.5 ${ }^{\circ}$, used in quadrants Q1, Q2, Q3) or a resistive anode (fine angular resolution, $2-5^{\circ}$, used in quadrant Q0) provide measurement of the azimuthal entry angle.

Combining the energy-per-charge $(E / Q)$, post-acceleration voltage (PAC), and timeof-flight $(\tau)$ measurements allows the determination of the mass-per-charge $(M / Q)$ of the incoming ion according to:

$$
M / Q=2 \cdot(E / Q+|P A C|) \cdot \alpha \cdot(\tau / d)^{2},
$$

where $d$ is the length of the time-of-flight path in the TOF/E Section and $\alpha(E, M)$ denotes the energy and species-dependent energy loss in the carbon foil. The mass $(M)$ of the ion is determined from the residual energy $E_{\mathrm{ssd}}$ and the time-of-flight $(\tau)$ measurement:

$$
M=2 \cdot E_{\mathrm{ssd}} / \beta \cdot(\tau / d)^{2},
$$


Table 1 Summary of PLASTIC section characteristics and basic measurements

\begin{tabular}{|c|c|c|c|c|c|}
\hline $\begin{array}{l}\text { Instrument } \\
\text { section }\end{array}$ & $\begin{array}{l}\text { Primary } \\
\text { measurement }\end{array}$ & $\begin{array}{l}\text { Energy range } \\
(E / Q)\end{array}$ & FOV & $\begin{array}{l}\text { Eff*Area }(\mathrm{SW}) \\
\text { or Eff*G }(\mathrm{ST})\end{array}$ & Time \\
\hline $\begin{array}{l}\text { SWS } \\
\text { Small } \\
\text { Channel }\end{array}$ & $\begin{array}{l}\text { Solar wind proton } \\
\text { bulk parameters } \\
\text { - } n \\
\text { - } V_{p} \\
\text { - } v_{-} t h \\
\text { Moments }\end{array}$ & $\begin{array}{l}0.3-10.6 * \mathrm{keV} / \mathrm{e} \\
\text { (* upper limit) } \\
6.3 \% \text { FWHM } \\
5 \% \text { Step spacing }\end{array}$ & $\begin{array}{l}\text { Centered on } \\
\text { Sun-Earth line } \\
45^{\circ} \times \pm 20^{\circ} \\
\text { (Dynamic) } \\
45^{\circ} \times 0.4^{\circ} \\
\text { (Instantaneous) }\end{array}$ & $\begin{array}{l}4 \times 10^{-4} \mathrm{~cm}^{2} \\
(\text { single } \\
\text { coincidence) } \\
2 \times 10^{-4} \mathrm{~cm}^{2} \\
\text { (double } \\
\text { coincidence) }\end{array}$ & $1 \mathrm{~min}$ \\
\hline $\begin{array}{l}\text { SWS } \\
\text { Main } \\
\text { Channel }\end{array}$ & $\begin{array}{l}\text { Solar wind minor } \\
\text { ions } \\
\text { - Species } \\
(M, M / Q) \\
\text { He to Fe } \\
\text { - Abundances } \\
\text { - Speeds } \\
\text { - Direction }\end{array}$ & $\begin{array}{l}0.3 *-80 \mathrm{keV} / \mathrm{e} \\
(* \text { lower limit) } \\
6.3 \% \mathrm{FWHM} \\
5 \% \text { Step spacing }\end{array}$ & $\begin{array}{l}\text { Centered on } \\
\text { Sun-Earth line } \\
45^{\circ} \times \pm 20^{\circ} \\
\text { (Dynamic) } \\
45^{\circ} \times 2^{\circ} \\
\text { (Instantaneous) }\end{array}$ & $\begin{array}{l}0.1 \mathrm{~cm}^{2} \\
\text { (double } \\
\text { coincidence) }\end{array}$ & $1-5 \mathrm{~min}$ \\
\hline $\begin{array}{l}\text { ST WAP } \\
\text { (w/SSD) }\end{array}$ & $\begin{array}{l}\text { Suprathermals } \\
\text { - Species } \\
(M, M / Q) \\
\text { H to Fe } \\
\text { - Flux } \\
\text { - Direction }\end{array}$ & $\begin{array}{l}0.3-80 \mathrm{keV} / \mathrm{e} \\
7.0 \% \mathrm{FWHM} \\
5 \% \text { Step spacing }\end{array}$ & $50^{\circ} \times 6^{\circ}$ & $\begin{array}{l}1 \times 10^{-3} \mathrm{~cm}^{2}- \\
\mathrm{eV} / \mathrm{eV}-\mathrm{sr} \\
\text { (single } \\
\text { coincidence) } \\
5 \times 10^{-4} \mathrm{~cm}^{2} \text { - } \\
\mathrm{eV} / \mathrm{eV}-\mathrm{sr} \\
\text { (double } \\
\text { coincidence) }\end{array}$ & $5-10 \mathrm{~min}$ \\
\hline $\begin{array}{l}\text { ST WAP } \\
\text { (noSSD) }\end{array}$ & $\begin{array}{l}\text { Suprathermals } \\
\text { - Species } \\
(M / Q) \\
\text { H to Fe } \\
\text { - Flux } \\
\text { - Direction }\end{array}$ & $\begin{array}{l}0.3-80 \mathrm{keV} / \mathrm{e} \\
7.0 \% \mathrm{FWHM} \\
5 \% \text { Step spacing }\end{array}$ & $\begin{array}{l}160^{\circ}(\mathrm{B}) / 175^{\circ} \\
(\mathrm{A}) \times 6^{\circ}\end{array}$ & $\begin{array}{l}3 \times 10^{-3} \mathrm{~cm}^{2}- \\
\mathrm{eV} / \mathrm{eV}-\mathrm{sr} \\
\text { (single } \\
\text { coincidence) } \\
2 \times 10^{-3} \mathrm{~cm}^{2} \text { - } \\
\mathrm{eV} / \mathrm{eV}-\mathrm{sr} \\
\text { (double } \\
\text { coincidence) }\end{array}$ & $5-10 \mathrm{~min}$ \\
\hline
\end{tabular}

The instrument cycles through $128 \mathrm{E} / Q$ voltage steps in a one-minute cadence

Values provided for detection efficiency times the energy-geometric factor or detection efficiency times the active area are representative values based a $2 \mathrm{keV} / \mathrm{e} \mathrm{He}^{+}$beam calibration with PAC set at $18-20 \mathrm{kV}$ and MCP bias set to $3000 \mathrm{~V}$ 


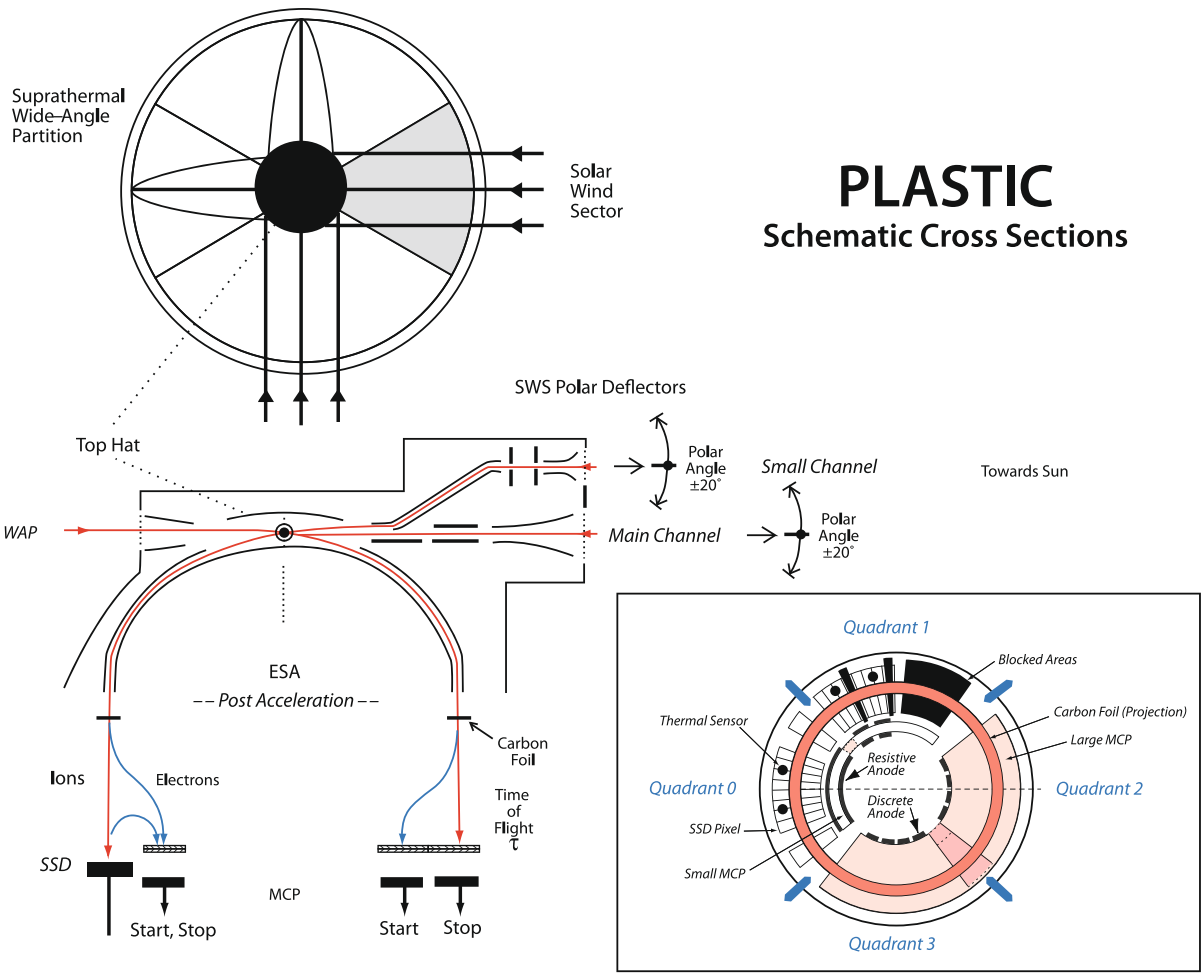

Fig. 2 PLASTIC Principle of Operation. (Top) View of sample ion trajectories through the ESA, looking down onto the entrance plane of the top-hat electrostatic analyzer (ESA). (Bottom) Cross-sectional schematic of the basic layout for the TOF $(M / Q)$ and TOF/E $(M / Q$ and $M)$ measurement (described in the text). (Insert) Schematic view, looking down into the TOF Chamber from the exit of the ESA, defining the four quadrants Q0, Q1, Q2, Q3 at the detector levels

where $\beta(E, M)$ is a function of the pulse height defect in the SSD over the incident particle energy. This defect is velocity and species dependent. Pre-flight calibrations and simulations determine the $\alpha$ and $\beta$ functions. This TOF plus $\mathrm{E}$ mass spectrometer technique has been successfully used by solar wind composition instruments flown on Ulysses (Gloeckler et al. 1992), on SOHO (Hovestadt et al. 1995), and on ACE (Gloeckler et al. 1998).

The following sections describe the details of the PLASTIC measurement components shown in the operational schematic (Fig. 2), as encountered by an incident particle.

\subsection{Entrance System / Energy Analyzer ( $E / Q$, Elevation Angle, and GF)}

\subsubsection{Entrance System / Energy Analyzer Overview}

The PLASTIC instrument measures solar wind and suprathermal ion distributions spanning a wide range of ion fluxes. By employing ion collection apertures with three different geometric factors (GF), the main solar wind distribution (protons and alphas), the less abundant solar wind heavy ion distributions, and selected types of suprathermal ion distributions can be measured within one instrument. An electrostatic deflection system called the Entrance System / Energy Analyzer (Allegrini 2002; Blush et al. 2005; 
Karrer 2007) selects solar wind ions for out-of-ecliptic angles of incidence and filters the solar wind and suprathermal ions by energy-per-charge $(E / Q)$ before the ions pass into the Time-Of-Flight/Energy (TOF/E) section of the instrument.

The Entrance System steers and filters ions utilizing the electrode configurations shown in Fig. 3. The sun-centered solar wind sector (SWS) spans a $45^{\circ}$ in-ecliptic FOV. The instantaneous polar angle acceptances are $0.4^{\circ}$ and $2^{\circ}$ FWHM for SWS Small and Main Channels, respectively, and up to $\pm 20^{\circ}$ out-of-ecliptic dynamic FOV is attained with the use of deflectors. The Small Channel (proton-alpha) and the Main Channel (heavy ion), positioned one above the other in the SWS (Fig. 1), collect the main distribution of solar wind ions.

The Wide-Angle Partition (WAP) spans the remainder of the $360^{\circ}$ in-ecliptic FOV (with the exclusion of spacecraft and instrument blockages). The WAP entrance aperture accepts ions in the polar direction with a FWHM of $3^{\circ}$ for a given $E / Q$, or about $6^{\circ} \mathrm{FOV}$ over the entire $E / Q$ bandwidth.

Ions collected through all entrance apertures are filtered by $E / Q$ with an electrode pair of toroidal domes that are used as an electrostatic analyzer (Ewald and Liebl 1955; Wollnik et al. 1972; Young et al. 1988). The ESA is cylindrically symmetric about the instrument azimuth, permitting the simultaneous collection of solar wind ions in the SWS and suprathermal ions in the WAP. The $E / Q$ bandwidth is $\sim 6 \%$ in the full $E / Q$ dynamic range, for all entrance apertures (Tables 2, 3 and 4).

The outer and inner domes of the ESA are connected, respectively, to the positive and negative high voltage power supplies SWEEP ESA_plus and SWEEP ESA_minus (Sect. 3.7.2). The voltages of both domes are matched and set simultaneously to one of 128 output settings (SWPE $=0-127$ ). These voltage settings are logarithmically spaced from $\pm 6000 \mathrm{~V}$ down to $\pm 10 \mathrm{~V}$, in order to cover the full $E / Q$ range from $\sim 80$ to $0.3 \mathrm{keV} / \mathrm{e}$.

The nominal energy-per-charge response of the ESA is given by

$$
E S A \_E / Q[\mathrm{keV} / \mathrm{e}]=0.2 \times 1.04895^{(127-\mathrm{SWPE})} .
$$

There is a non-linear response in the power supplies at the lowest voltages, bringing the lowest $E S A \_E / Q$ closer to $0.3 \mathrm{keV} / \mathrm{e}$ rather than the nominal value of $0.2 \mathrm{keV} / \mathrm{e}$. The voltage settings are loaded into a table for implementation. These table entries can be changed by ground command.

During a 60-second collection cycle, comprised of 128 ESA steps, the ESA voltages are stepped and held every $435.6 \mathrm{~ms}$, which entails a $26 \mathrm{~ms}$ interval for HV settling followed by $409.6 \mathrm{~ms}$ of data accumulation. At each step of the ESA voltages, the polar angle deflectors in the SWS are ramped through a pre-defined set of voltages provided by the deflector high voltage power supplies (SWEEP DEFL1, SWEEP DEFL2), providing 32 deflection bins per ESA step, with an accumulation time of $12.8 \mathrm{~ms}$ per deflection bin. The voltages of the deflectors are matched to each set of ESA voltages in order to collect incident ions up to $\pm 20^{\circ}$ in elevation, with each DEFL bin nominally corresponding to about $1.25^{\circ}$ in polar angle. After the ESA cycle has been completed ( $\sim 56 \mathrm{sec})$, the remaining time in the 60-second cadence is used for sampling housekeeping, data transfer, resetting registers, implementing commands, and setting up the SWEEP high voltages for the start of the next collection cycle.

The Solar Wind Sector Main and Small Channels are activated sequentially, with the switching between channels commanded by the IDPU based upon a monitored rate count. The less-abundant solar wind ion species (typically $<1 \%$ of the solar wind) are collected in the initial part of the ESA stepping cycle though the Main Channel, which has a large energy-geometric factor $\left(G_{\left(45^{\circ}\right)} \sim 2.5-3.2 \times 10^{-3} \mathrm{~cm}^{2} \mathrm{sr} \mathrm{eV} / \mathrm{eV}\right.$ per $45^{\circ}$, active area 


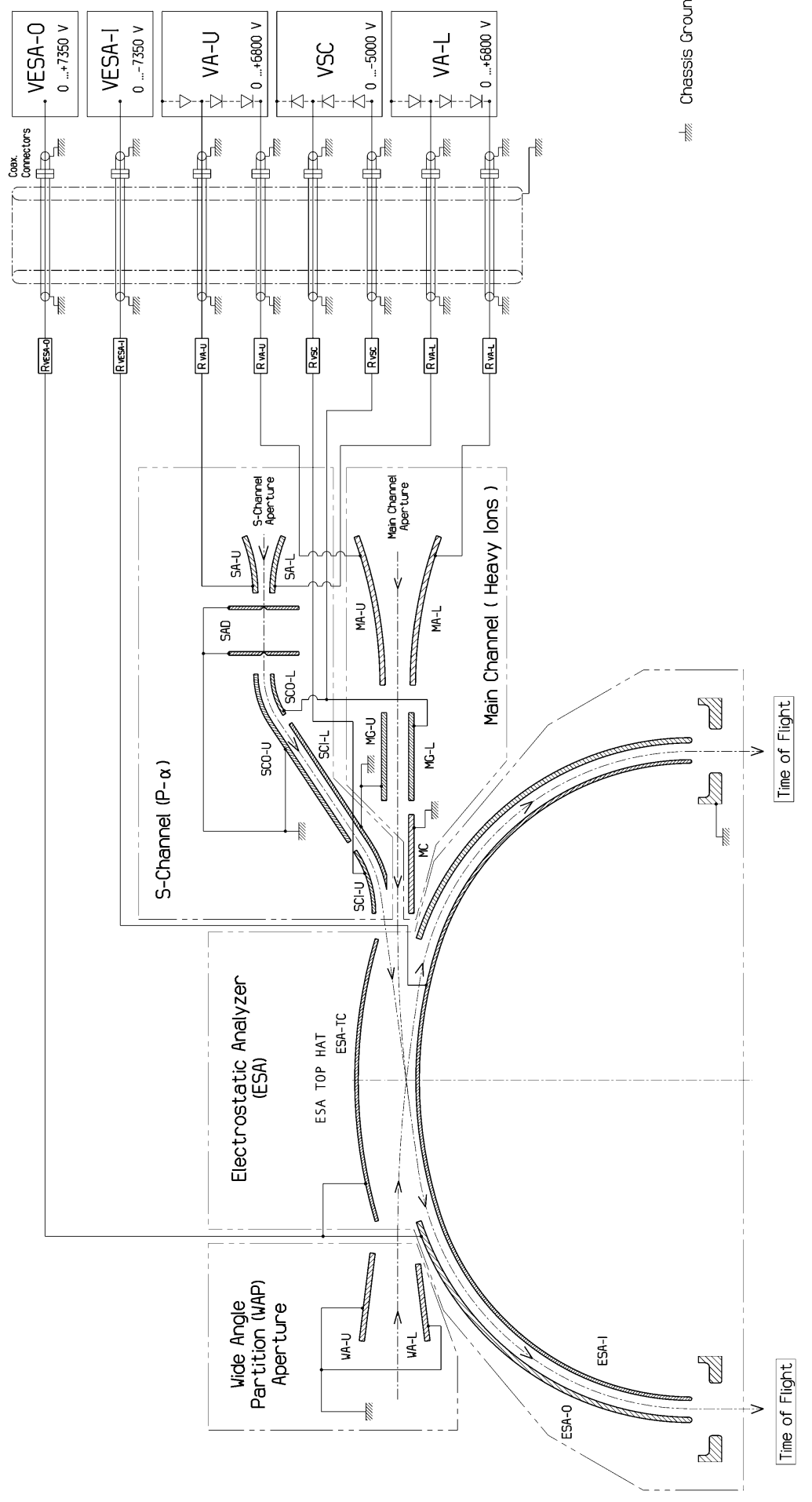

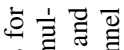

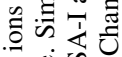
过 这 论

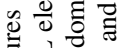

昰过富 脝文

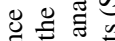
든

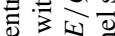
Ф 氜 氖 บ己 즐 芯可氙 之氖 踏 胥 c) 00 क

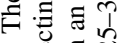
घं 당 क 福主

밀 है 氠已包

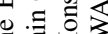
$\equiv$ 元 पั口 过 के 定远 윰 000 $\circ \cong$

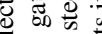
๑. 苛志 D . \& 4 毛定 守论 के .

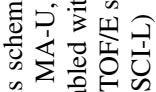

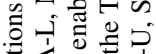

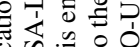

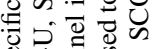

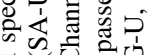
बढ U . 을 跣 ه 0 象象要

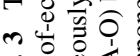

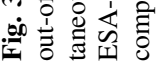


$A_{\text {act }} \sim 0.8 \mathrm{~cm}^{2}$ ). As the ESA voltage decreases, the incident ion flux increases as the solar wind alpha particle (typically $\sim 4 \%$ in number density) and then proton (typically $\sim 96 \%$ in number density) portions of the distribution are sampled. At a predetermined (commandable) count rate set point, the SWEEP SCHN high voltage is enabled and applied to the steering electrodes (SCO-L, SCI-U), allowing ions to pass through this channel into the ESA. These SCHN voltages must be appropriately tuned to the ESA settings. Simultaneously, an electrostatic deflection gate (Main Gate, MG) located past the Main Channel entrance aperture disables ion collection through the Main Channel. This effectively switches the geometrical factor to the smaller active area Small Channel $\left(G_{\left(45^{\circ}\right)} \sim 6 \times 10^{-7} \mathrm{~cm}^{2} \mathrm{sr}\right.$ $\mathrm{eV} / \mathrm{eV}, A_{\text {act }} \sim 1.5 \times 10^{-3} \mathrm{~cm}^{2}$ ). This GF switching is necessary to prevent saturation of the detector electronics and to limit the lifetime fluence on the SSD and MCP detectors.

In addition to the instrument ion measurement properties, the Entrance System suppresses solar UV radiation. All electrode surfaces are blackened with copper-sulfide and many surfaces are serrated. The instrument is designed to minimize direct exposure of internal components to solar photospheric UV radiation; pre-launch measurements give a UV suppression factor exceeding $1 \times 10^{8}$. The serrated surfaces in the Entrance System also inhibit scattered particles from reaching the TOF/E Chamber.

\subsubsection{Characterization of the Entrance System / Energy Analyzer}

As a major subsystem of the PLASTIC instrument, the Entrance System has undergone extensive design, prototype testing, and flight model calibrations prior to integration. Considering the complex ion optical system of the PLASTIC instrument, calibration and simulation of the sub-system responses are important for understanding the overall instrument behavior. The calibration results herein summarized are details of the final Flight Model (FM-A, FM-B) calibrations, which are fully presented in Karrer (2007). Karrer (2007) also presents details of the Flight Spare Entrance System (not flown).

The Entrance System ion optical calibration measurements were performed in the CASYMS ion beam facility at the University of Bern (Ghielmetti et al. 1983; Steinacher et al. 1995). CASYMS provides a broad parallel beam of an area of $\sim 250 \mathrm{~cm}^{2}$ of uniform flux. CASYMS delivers ions of many different species and charge states in an energy range from $5 \mathrm{eV} / \mathrm{e}$ to $60 \mathrm{keV} / \mathrm{e}$, with an energy spread of $\sim 0.1 \%(<1 \%$ for very low $E / Q$ ) and a beam divergence of $\sim 0.3^{\circ} . \mathrm{Ar}^{+}$and $\mathrm{He}^{+}$beams in an energy range from $0.1 \mathrm{keV} / \mathrm{e}$ to $60 \mathrm{keV} / \mathrm{e}$ were used for the Entrance System calibration. The systems were mounted on a turning table with elevation and azimuth angle articulation. (Note that the CASYMS elevation angle corresponds to the solar wind out-of-ecliptic angle.) Ions passing though the ESEA were counted by micro-channel plates in chevron configuration with a position-sensing anode (Lampton and Carlson 1979). Variation in detection efficiency due to particle energy was accounted for in the results (see Allegrini 2002; Karrer 2007).

The energy acceptance and the analyzer constants were determined using different beam energies and ion species. The analyzer constants give the relation between the applied electrode voltage and the collected ion energy per charge, $E / Q$ :

$$
k_{\text {electrode }}=E / Q / V_{\text {electrode }} \text {. }
$$

The measured values given in Table 2 are quoted in terms of eV/e per volt, reflecting the energy-per-charge units (eV/e) normally used in describing an ESA response. The analyzer constant given for the Small Channel differs slightly from the rest, because ions passing 
Table 2 Overview of entrance system analyzer constants

\begin{tabular}{|c|c|c|c|c|c|}
\hline \multirow{2}{*}{$\begin{array}{l}\text { Entrance } \\
\text { system } \\
\text { channel }\end{array}$} & \multirow{2}{*}{$\begin{array}{l}\text { Electrode } \\
\text { identifier }\end{array}$} & \multicolumn{2}{|c|}{ ES/ESA-FM A } & \multicolumn{2}{|c|}{ ES/ESA-FM B } \\
\hline & & $\begin{array}{l}\text { Analyzer } \\
\text { constant } \\
\left(e^{-} / \mathrm{e} \mathrm{V}^{-1}\right)\end{array}$ & FWHM & 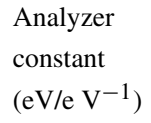 & FWHM \\
\hline \multirow[t]{3}{*}{ Small } & SCO-L & $3.23(1)$ & $10.40 \%$ & $3.25(1)$ & $10.64 \%$ \\
\hline & SCI-U & $3.68(1)$ & $13.20 \%$ & $3.64(1)$ & $10.75 \%$ \\
\hline & ESA & $8.46(1)$ & $6.35 \%$ & $8.46(2)$ & $6.26 \%$ \\
\hline Main & ESA & $8.26(1)$ & $6.12 \%$ & $8.26(2)$ & $6.48 \%$ \\
\hline WAP & ESA & $8.25(4)$ & $6.77 \%$ & $8.26(1)$ & $7.30 \%$ \\
\hline
\end{tabular}

Table 3 Overview of deflection constants and angular acceptance

\begin{tabular}{|c|c|c|c|c|}
\hline \multirow{2}{*}{$\begin{array}{l}\text { Entrance } \\
\text { system } \\
\text { channel }\end{array}$} & \multicolumn{2}{|l|}{ ES/ESA-FM A } & \multicolumn{2}{|l|}{ ES/ESA-FM B } \\
\hline & $\begin{array}{l}\text { Deflection } \\
\text { constant } \\
\left(\mathrm{deg}-\mathrm{keV} / \mathrm{e} \mathrm{V}^{-1}\right)\end{array}$ & $\begin{array}{l}\text { Angular FWHM } \\
\text { (deg) }\end{array}$ & $\begin{array}{l}\text { Deflection } \\
\text { constant } \\
\left(\mathrm{deg}-\mathrm{keV} / \mathrm{e} \mathrm{V}^{-1}\right)\end{array}$ & $\begin{array}{l}\text { Angular FWHM } \\
\text { (deg) }\end{array}$ \\
\hline Small & $0.117(1)$ & 0.37 & $0.114(1)$ & 0.27 \\
\hline Main & $0.128(3)$ & 1.9 & $0.127(3)$ & 1.8 \\
\hline WAP & - & 3.2 & - & 3.1 \\
\hline
\end{tabular}

through the Small Channel enter the ESA at a slight angle $\left(\sim 0.2^{\circ}\right)$ from above the instrument plane (Fig. 3), thereby lowering the voltage required to collect an ion of a given energy. The energy acceptance is given by the FWHM of the energy distribution as measured by varying the ESA voltage for a given beam energy.

The angular acceptance and deflection constants for the deflection plates were determined using different energies and elevation angles (see Table 3 ). The deflection constants give the relation between applied electrode voltage and the ion incident angle:

$$
k_{\text {deflection }}=\Theta \cdot E / Q / V_{\text {electrode }}
$$

where $\Theta$ is the polar (elevation) angle, $E / Q$ is the beam energy per charge, and $\mathrm{V}_{\text {electrode }}$ is the voltage of the deflection electrode. The angular acceptance is given by the FWHM of the angular distribution measured by varying the elevation angle for a given beam energy.

To determine the geometric factor, the instrument electrodes were tuned for a specific beam energy after which the active areas, $A_{a c t}$, were measured for different beam energies and elevation angles:

$$
A_{a c t}=n_{\text {detector }}\left(\Phi t \eta_{\text {detector }}\right)^{-1}
$$

where $n_{\text {detector }}$ is the counts on the detector during the accumulation time $t, \Phi$ is the beam flux per unit area, and $\eta_{\text {detector }}$ is the detection efficiency of the particle detector. The results are plotted in Fig. 4 in terms of the angular and energy acceptance for the three different entrance apertures of FM A and FM B. The respective geometric factors $G$ (similar to the energy-geometric factor $G_{\mathrm{E}}$ as defined by Young et al. 1989), were calculated from the 
Table 4 Active areas and geometrical factors for the FM-A and FM-B Entrance System Apertures

\begin{tabular}{|c|c|c|c|c|c|}
\hline \multirow{2}{*}{$\begin{array}{l}\text { Entrance } \\
\text { system } \\
\text { channel }\end{array}$} & \multirow{2}{*}{$\begin{array}{l}\text { Azimuth } \\
\text { position } \\
\text { (deg) }\end{array}$} & \multicolumn{2}{|l|}{ FM A } & \multicolumn{2}{|l|}{ FM B } \\
\hline & & $\begin{array}{l}\text { Active area } \\
\left(\mathrm{cm}^{2}\right)\end{array}$ & $\begin{array}{l}\text { Geometrical } \\
\text { factor } G \\
\left(\mathrm{~cm}^{2} \mathrm{eV} / \mathrm{eV} \mathrm{sr}\right. \\
\left.\text { per } 45^{\circ} \text { sector }\right)\end{array}$ & $\begin{array}{l}\text { Active area } \\
\left(\mathrm{cm}^{2}\right)\end{array}$ & $\begin{array}{l}\text { Geometrical } \\
\text { factor } G \\
\left(\mathrm{~cm}^{2} \mathrm{eV} / \mathrm{eV} \text { sr }\right. \\
\left.\text { per } 45^{\circ} \text { sector }\right)\end{array}$ \\
\hline Small & 0 & $1.41(4) \times 10^{-3}$ & $6.0(5) \times 10^{-7}$ & $1.50(4) \times 10^{-3}$ & $6.3(4) \times 10^{-7}$ \\
\hline Main & 0 & $8.9(7) \times 10^{-1}$ & $3.15(4) \times 10^{-3}$ & $7.5(5) \times 10^{-1}$ & $2.52(4) \times 10^{-3}$ \\
\hline WAP & $\begin{array}{l}-90 \\
-180 \\
-38.5\end{array}$ & $\begin{array}{l}8.2(3) \times 10^{-1} \\
1.12(8) \\
4.7(2) \times 10^{-1}\end{array}$ & $\begin{array}{l}8.35(6) \times 10^{-3} \\
6.82(7) \times 10^{-3} \\
3.19(3) \times 10^{-3}\end{array}$ & $\begin{array}{l}- \\
9.4(4) \times 10^{-1} \\
-\end{array}$ & $\begin{array}{l}- \\
6.44(6) \times 10^{-3} \\
-\end{array}$ \\
\hline
\end{tabular}

discrete measurements using the approximation:

$$
G_{\left(45^{\circ}\right)} \approx \alpha_{S W S} \cdot \sum_{i} \sum_{j} \Delta \theta_{i}\left(\Delta E_{j} / E_{j}\right)\left(A_{a c t}\right)_{i j}
$$

where $\alpha_{S W S}$ is an integrated (over $45^{\circ}$ ) azimuth angle parameter, $\Delta \theta_{i}$ is the elevation angle step size for step $i, E_{j}$ and $\Delta E_{j}$ energy and energy step size for step $j$, and $\left(A_{a c t}\right)_{i j}$ is the active area of step $i, j$.

The discrete-sums approximation for calculating the geometric factor as applied to the PLASTIC entrance system is discussed by Allegrini (2002) and by Karrer (2007). The $\alpha_{S W S}$ parameter incorporates a full integration in azimuth over $45^{\circ}$, under the assumption of uniform response and cylindrical symmetry. The measured active areas and geometric factors for each entrance system and its apertures are provided in Table 4. The active area, sometimes called the directional geometric factor, is defined for a parallel beam, and is useful for solar wind applications. Note that the geometrical factors in Table 4 appear different from those provided in Table 1. The entries specified in Table 1 were obtained from the integrated instruments, and include the effects of transmission in the TOF/E Chamber, the efficiency of the detectors (Sect. 3.4.3), and the full FOV of the respective channel.

For the Small Channel of both flight models, a complete elevation versus azimuth angle scan of the system response was performed (Fig. 5a,b). An azimuth angle scan for one elevation angle was made for the Main Channel and WAP (Fig. 5c,d). For these measurements all electrodes were tuned for an incident beam energy $E / Q=3 \mathrm{keV} / \mathrm{e}$.

Entrance System calibration and testing (including a full set of environmental tests) has ensured optimal ion optical functionality after integration with the full PLASTIC. Detailed knowledge of the response functions, especially the analyzer and deflection constant relations between applied voltage and measured ion properties, are critical for setting in-flight operational tables for the ESA, Channels, and Deflectors ("SWEEP") high voltage system, and ultimately for the interpretation of the resulting solar wind data.

\subsection{Time-of-Flight Measurement System}

\subsubsection{TOF/E Chamber Overview}

An ion exiting the ESA is accelerated through a $-15 \mathrm{kV}$ to $-25 \mathrm{kV}$ voltage drop (PAC voltage, commandable) just before entering the TOF/E chamber (refer to Fig. 2). The ion 


\section{Entrance System Response \\ Entrance System Response}

FM-A

a)

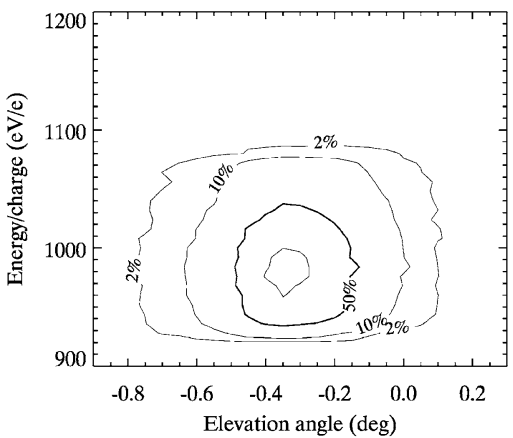

c)

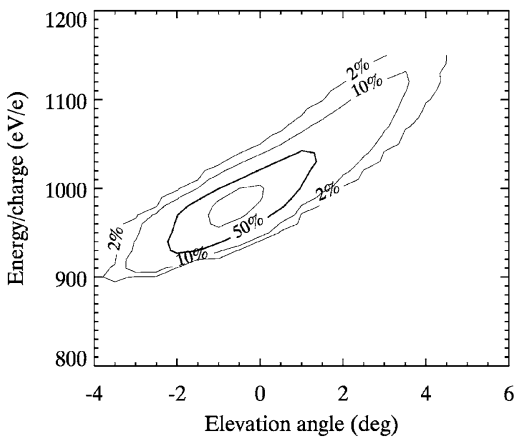

e)

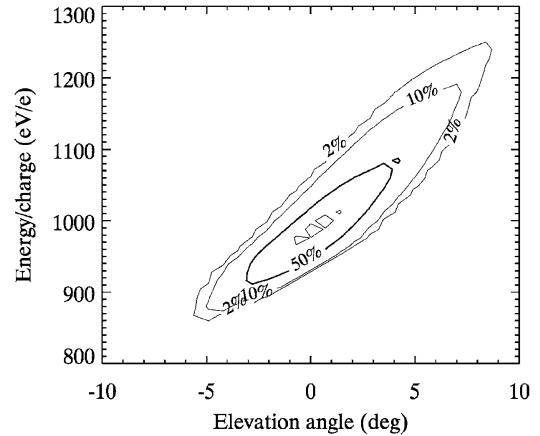

FM-B

b)

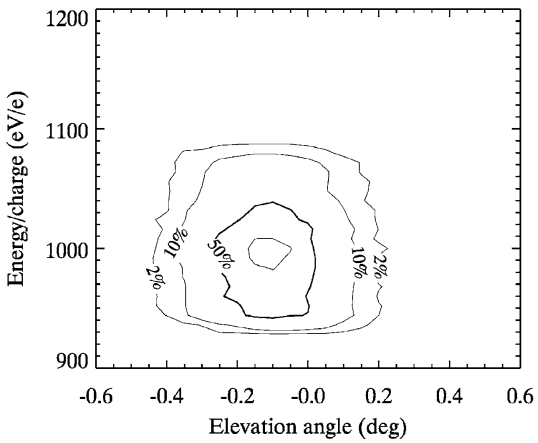

d)

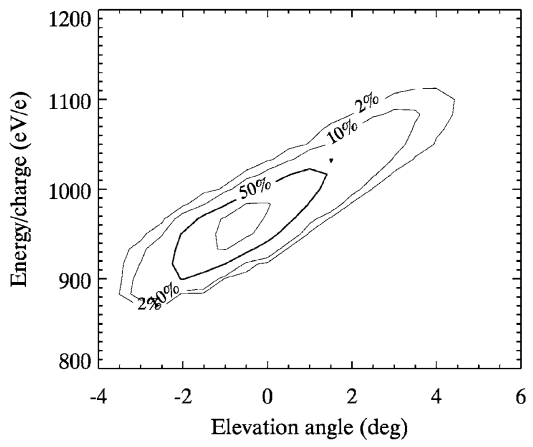

f)

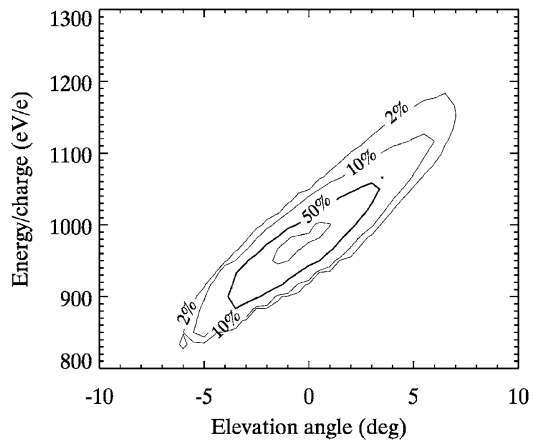

Fig. 4 Contours of relative geometrical factors. (a, b) Small Channel on the FM-A and FM-B Entrance Systems as a function of beam energy and elevation angle (all normalized to $1 \mathrm{keV} / \mathrm{e}$ ). For FM-A, the ion beam used was $5 \mathrm{keV} / \mathrm{e} \mathrm{He}{ }^{+}$. For FM-B, $5 \mathrm{keV} / \mathrm{e} \mathrm{Ar}^{+}$was used. (c, d) Main Channel on the FM-A and FM-B Entrance Systems as a function of beam energy and elevation angle (all normalized to $1 \mathrm{keV} / \mathrm{e}$ ). For FM-A, the beam was $5 \mathrm{keV} / \mathrm{e} \mathrm{Ar}^{+}$. For FM-B, $3 \mathrm{keV} / \mathrm{e} \mathrm{Ar}^{+}$was used. (e, f) WAP Channel on the FM-A and FM-B Entrance Systems as a function of beam energy and elevation angle (all normalized to $1 \mathrm{keV} / \mathrm{e}$ ). For FM-A, the beam used was $5 \mathrm{keV/e} \mathrm{Ar}^{+}$. For FM-B, $3 \mathrm{keV/e} \mathrm{Ar}^{+}$was used 
Entrance System Response

FM-A

a)

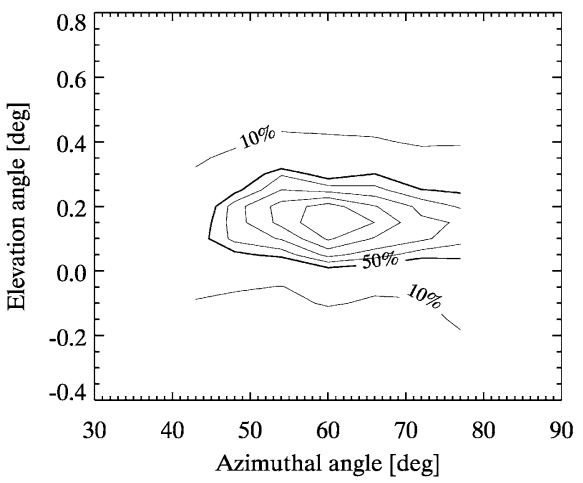

c)

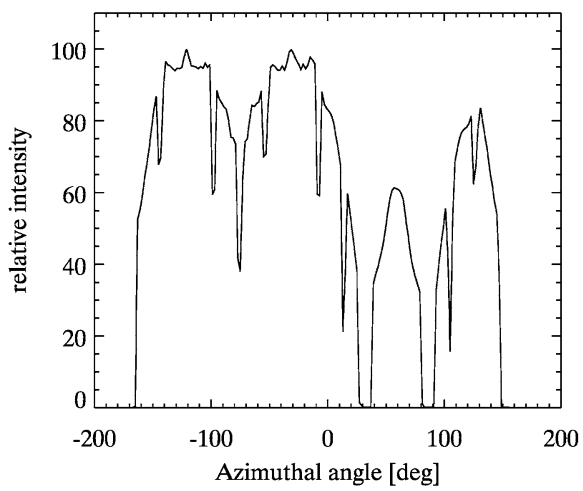

Entrance System Response

FM-B

b)

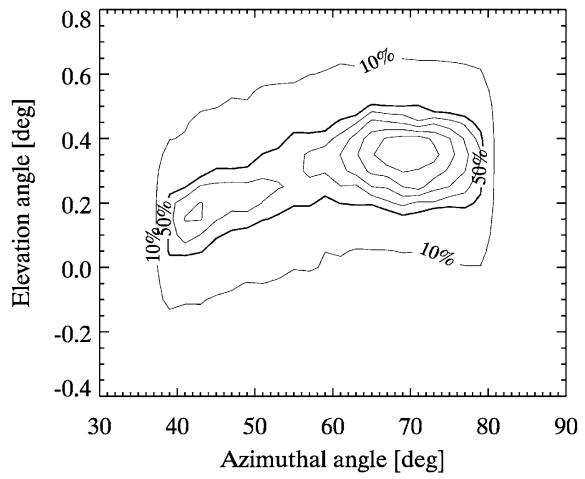

d)

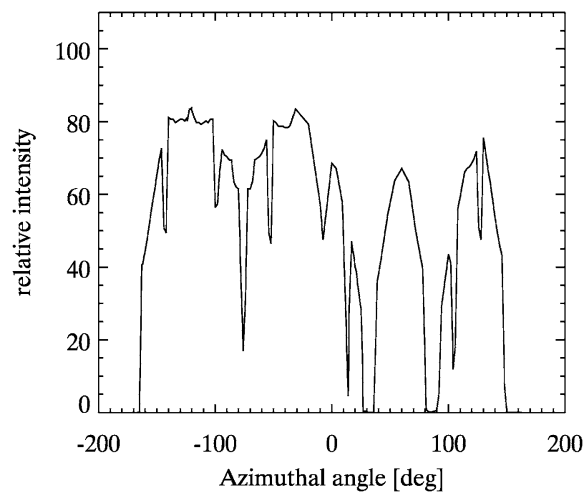

Fig. 5 Azimuth angle scans. Center of SWS in the calibration coordinate system is at $60^{\circ}$. (a, b) Azimuth response as a function of elevation angle for the Small Channel, for Entrance Systems FM-A and FM-B, respectively. (c, d) Azimuth response for the Main Channel and the WAP at a single elevation angle: -0.5 deg for FM-A and 0 deg for FM-B. Intensity differences are due to different beam settings used between FM-A and FM-B

then traverses a thin $\left(\approx 3.5 \mu \mathrm{g} / \mathrm{cm}^{2}\right)$ carbon foil located at the entrance to the chamber. The carbon foil is deposited on a supporting nickel grid that, together with an associated overlying field control grid (for suppression of backscattered electrons), is $83 \%$ transparent to the incident particles. Grid transmission was measured optically as well as with an ion beam (Lüthi 2003).

The carbon foil emits secondary electrons (Meckbach et al. 1975) that are subsequently accelerated and steered by guiding electrodes onto a micro-channel plate (MCP) chevron pair to trigger the "START" timing signal. The cascade (output) electrons at the exit of the MCPs are accelerated from the negative PAC voltage of the TOF/E Chamber to the signal board at ground potential. A fast "START" signal is read from an $80 \%$ transmission grid, and the "START" position is read from anodes located below the grid. 
After the ion traverses the carbon foil, it continues its passage through the TOF/E chamber and reaches the detector level, where it impacts either a solid state detector (SSD) or the "STOP" portion of a large MCP. The nominal (without scattering) travel distance is $8.0 \mathrm{~cm}$ on the SSD level, and $5.8 \mathrm{~cm}$ on the "Large" MCP detector level.

In the SSD section, the penetrating particle causes the emission of backscattered secondary electrons from the detector surface. These electrons are steered onto a "Small" MCP. The cascade electrons at the exit of the MCP are accelerated from the negative PAC voltage of the TOF/E Chamber to the signal board at ground potential. The fast "STOP" signal is provided from a solid anode located on the signal board.

In portions of the WAP sector where there are no SSDs, the incident particle directly impacts the "Large" MCP, thereby triggering its "STOP" timing signal directly.

The START and STOP signals of the SSD and non-SSD sections of PLASTIC are fed into two independent time-to-amplitude converter (TAC) boards. Rate information is provided from the START, STOP, and Coincidence (START with STOP) elements of the two sections, as is a measurement of the flight time $\tau$ of the ions. The time signals are processed in a 10-bit time-to-amplitude converter. The $\tau$ range $\sim 4$ to $250 \mathrm{~ns}$ is converted to TOF channel range 0 1023; and the TOF value is subsequently transferred to the event selection logic for further processing (see Sect. 3.7). These two boards (designated TAC0 and TAC2) also provide in-flight test and calibration capabilities by adjustable internal stimulation circuitry. The electronic resolution of the time measurement is $<0.2 \mathrm{~ns}$. The total time-of-flight dispersion is also affected by the angular spread and energy straggling of the ion passage through the carbon foil.

\subsubsection{Design of the TOF/E Chamber}

A cutaway representation of the PLASTIC Entrance System and TOF/E Chamber is shown in Fig. 6. The TOF with energy measurement system is shown on the right half of the TOF/E Chamber, and the TOF-only measurement system on the left side of the cutaway. The TOF/E chamber dimensions and the applied electrode voltages were designed using the electrostatic ion optics analysis and design program SIMION 3D version 7.0 (Dahl 1995). This program calculates ion and electron trajectories, time-of-flight, kinetic energy, angles of incidence, and electric potential field arrays. The program output was used in designing the SSD, MCP, and anode detector dimensions and their respective placement in the TOF/E Chamber, in order to maximize the probability of hitting the targeted detector (i.e., optimization of the geometrical factor). Simulations were also used in assigning the voltages applied to the steering and focusing electrodes and to the electron acceleration grids. Electrode and grid parameters were varied until the output response was optimized to minimize the dispersion in the time-of-flight (species resolution) and in the angle (position integrity and resolution). The fidelity of the simulation relies on appropriate initial inputs. User inputs include the initial particle distributions (species, energy, position, elevation and azimuth angles) for: (1) ions exiting the carbon foil; (2) secondary electrons from the carbon foil and from the SSD; and (3) cascade (MCP output) electrons for start and stop signals.

The method used in generating the test file for ions exiting the carbon foil is fully described by Allegrini (2002). The standard run assumed nominal solar wind element abundances and charge state distributions (1.5 MK freeze-in temperature) for $\mathrm{O}, \mathrm{Ne}, \mathrm{Mg}, \mathrm{Si}, \mathrm{Ar}$, and $\mathrm{Fe}$, representing a range of solar wind $Z>2$ species; a bulk speed of $440 \mathrm{~km} / \mathrm{s}$ with nominal thermal spread; post acceleration of $-25 \mathrm{kV}$; and carbon foil charge exchange, energy loss, and angle dispersion. The carbon foil energy loss and angular straggling functions were measured by Lüthi (2003) for the same $3.5 \mu \mathrm{g} / \mathrm{cm}^{2}$ carbon foils and supporting grid 
Fig. 6 Cutaway view of the PLASTIC Entrance System and TOF/E Chamber

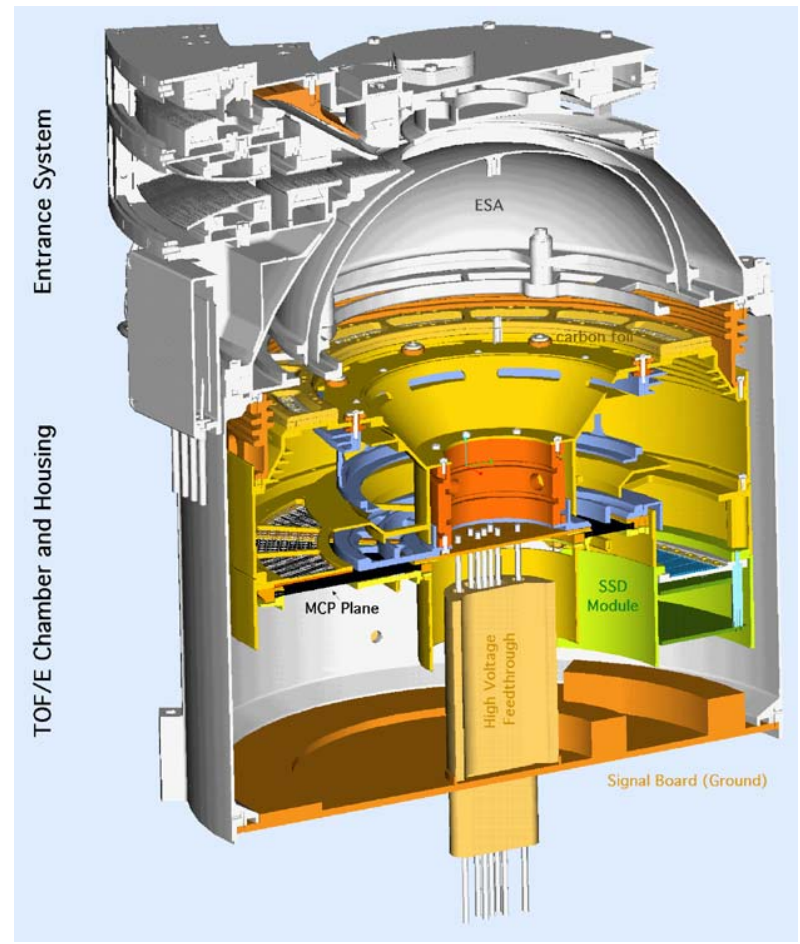

system as those used in PLASTIC. Charge exchange within the foil is expected to alter the ion's ionic charge state, and the results of Gonin et al. (1995) were used for determining the post-foil charge fractions. Separate simulation runs were executed for singly charged species, as would represent solar wind protons or oxygen pickup ions.

Secondary electrons emitted from solids typically have an energy distribution with a maximum (most probable energy) of $\sim 2-3 \mathrm{eV}$, with a long energy tail, and a cosine angular distribution relative to the surface normal (e.g., Wehner 1966; Kawata et al. 1991; Ritzau and Baragiola 1998). This general distribution shape was used in generating the simulated test electrons exiting from the carbon foil and from the SSD surface. For the simulated cascade (MCP output) electrons, the energy distribution was derived from Koshida and Hosobuchi (1985), with the initial electron angular dispersion centered at the MCP channel angle $\left(19^{\circ}\right.$ for the large $\mathrm{MCP}, 13^{\circ}$ for the small MCP).

Figure 7 illustrates the simulated trajectories for the Solar Wind Sector side of the TOF/E Chamber using these input distributions. Ions are shown exiting the carbon foil and hitting the solid state detector; start electrons are steered from the carbon foil to the small micro-channel plate; stop electrons emitted from the front surface of the SSD are steered to the small micro-channel plate; and the MCP-generated cascade electrons are accelerated to their respective anodes located on the signal board. Combined target efficiency for nominal operational voltages was estimated (through these simulations) to be $>92 \%$.

Simulations were also employed to verify that any backscattered electrons, created either by ions or electrons hitting internal structures other than their intended detectors, would not accidentally be steered onto an MCP, where it could trigger false event counts (creating a background population). 
Fig. 7 Ion and electron trajectories from a SIMION 3D simulation for the TOF/E chamber on the Solar Wind Sector side (with SSDs)

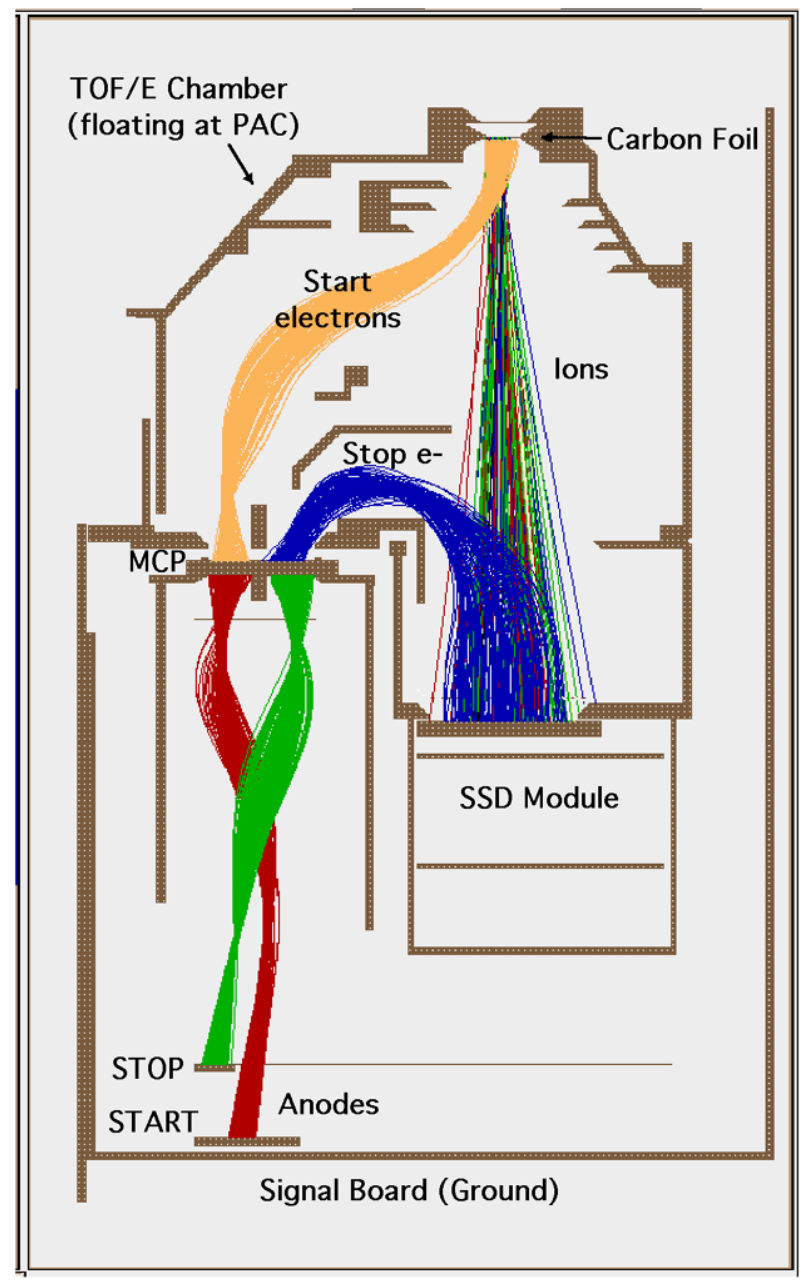

Prototype testing was performed using the UNH ion gun facility $(0-40 \mathrm{keV} / \mathrm{e}$, singly charged ions) to verify the simulation results and to determine any modifications that were needed (e.g., for fringe field effects). An over-sized square MCP with a position-sensing anode (Lampton and Carlson 1979) was used in the prototype tests to verify geometries and placements chosen for the various detectors. The laboratory MCP could be rotated in the detector plane (i.e., in azimuth) to determine relative detection efficiencies as a function of position. Laboratory power supplies were used to determine the fine adjustment of various steering and grid voltages on the prototype. End-to-end performance testing was performed on the integrated engineering and flight models.

Some typical species resolution for the fully integrated PLASTIC Flight Model is illustrated in Fig. 8a. These calibration data for protons and heavy ions were taken at the CASYMS facility. 
Fig. 8a TOF distributions obtained for $\mathrm{H}^{+}, \mathrm{He}^{+}$, and $\mathrm{Ne}^{+4}$ ions at 1 and $60 \mathrm{keV} / \mathrm{e}$ CASYMS (UBe) beam energies. TOF resolution is a function of the species and total energy of the ion. Higher charge states for a given species will have higher energies, and hence improved resolution, as seen in the response for the $\mathrm{Ne}^{+4}$ ions
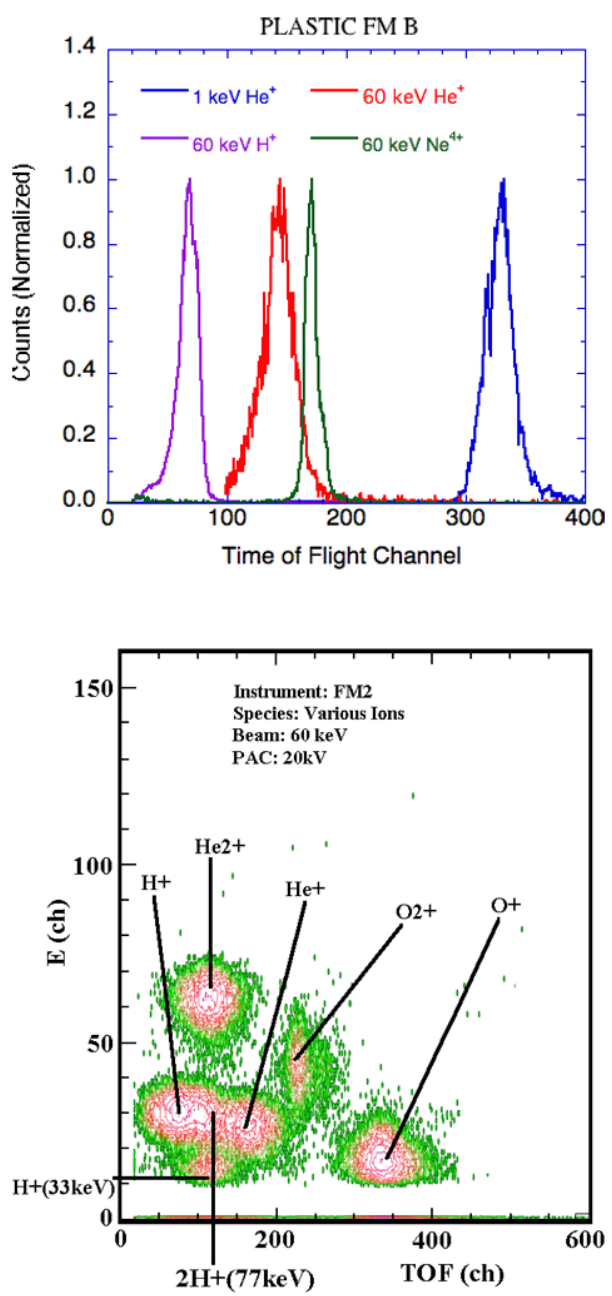

Fig. 8b A composite of Energy vs. TOF obtained for various ions, illustrating resolution for different species

\subsubsection{TOF Detection Efficiency}

The total efficiency for measuring the particular ion in the TOF/E system is affected by three separate contributions: the efficiency for getting a "START" signal, the efficiency for getting a "STOP" signal, and the efficiency for getting a "VALID EVENT".

The START efficiency is a function of the number of secondary electrons emitted from the carbon foil; the effectiveness of focusing these electrons onto the START MCP; the MCP detection efficiency; and the anode detection efficiency and signal threshold. On the "small" MCP side, the STOP efficiency is a function of the scattering of the ion in the carbon foil (that is, the number of ions successfully hitting the active area of the SSDs); the yield of electrons emitted from the SSD; the focusing of these electrons onto the STOP side of the $\mathrm{MCP}$; the MCP detection efficiency; and the corresponding anode detection efficiency and signal threshold. On the "large" MCP side, the STOP efficiency has similar inputs, except the ion itself triggers the STOP MCP. The VALID EVENT efficiency depends on the logic conditions imposed on the event selection. These typically include the requirement for a 
Fig. 8c Energy vs. TOF for an argon beam with various ionic charge states, illustrating the charge state resolution within a single species

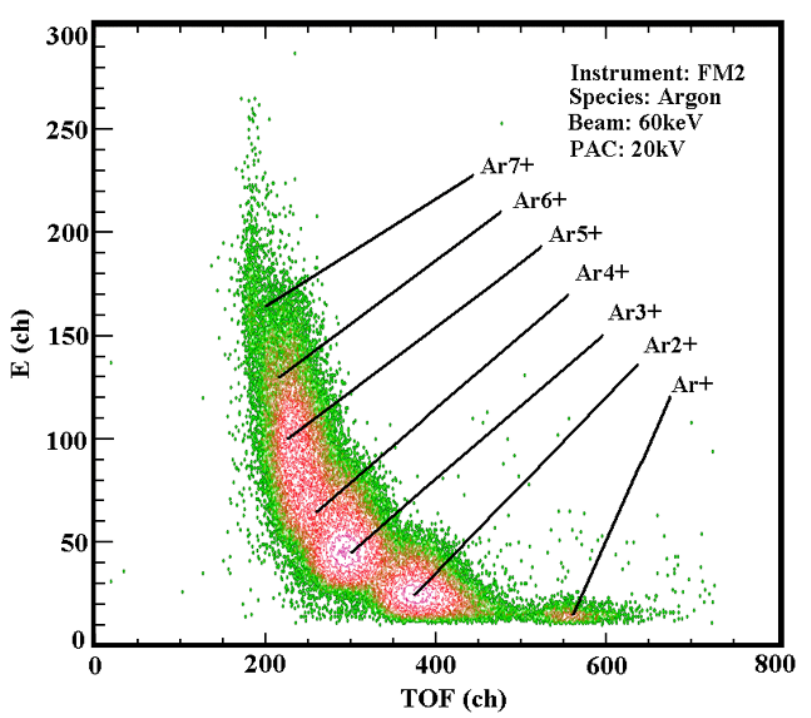

START signal and a coincident STOP signal (thereby providing a double coincident TOF), and may include conditions on energy and position coincidences.

The yield of secondary electrons forward emitted from the carbon foil or backward emitted from the SSD surface is a function of the incident ion's mass and velocity. The yield is typically larger for forward emission than backward emission, with smaller yields for protons than for heavier ions at a given velocity (Meckbach et al. 1975; Ritzau and Baragiola 1998).

The effectiveness of the focusing of the secondary (and the cascade) electrons were simulated and then tested with a prototype, as discussed previously. The simulations also included the measured effect of carbon foil straggling (Lüthi 2003).

The detection efficiency of MCPs is a strong function of its gain, which in turn depends upon its dimensions (active area, plate thickness and channel diameter), bias voltage, and the type, energy and angle of the primary radiation or particle being detected (Hellsing et al. 1985; Photonis, private communication). The annular, long-life micro-channel plates (MCP) used in PLASTIC were custom made by Photonis, and were provided as matched (in resistance) chevron pairs. The channel bias angle for the "small" MCPs was set at $13^{\circ}$ with an azimuth orientation. This angle was chosen to optimize the gain for incident electrons (which in this case are used for both the START and the STOP signals), while limiting the likelihood of ion feedback. For the "large" MCP, the START particles are secondary electrons, while the STOP particle is the incident ion itself. For this configuration, the channel bias angle was set to $19^{\circ}$, with a radial orientation. (The expected angles of incidence and the angular spread for the particles impacting the MCP were determined from the TOF/E Chamber simulations.) The gain and pulse height distribution as a function of bias voltage was established for each MCP chevron pair using an americium-241 alpha source and a nickel-63 beta source. These measurements were taken at the UBe KAFKA facility, and the results were used to make the flight unit selections.

The UNH ion gun and the UBe CASYMS ion beam calibration facilities were used to determine the final (end-to-end) efficiencies of the integrated instruments. The calibrations were achieved in two steps. First, the relative efficiency was measured for selected ion species as a function of the MCP bias. Second, using the optimum MCP voltage, the effi- 
ciencies for selected species were measured as a function of energy and position. Calibration data were obtained for beams of $\mathrm{H}^{+}, \mathrm{H}_{2}^{+}, \mathrm{He}^{+1,+2}, \mathrm{~N}^{+}, \mathrm{O}^{+1,+2}$, and $\mathrm{Ar}^{+1,+2,+3,+4,+5,+6}$. Figure 9 illustrates the START, STOP, and VALID EVENT efficiencies measured for Neon and Argon in quadrant Q0 of the PLASTIC FM2/B. In Fig. 10, proton STOP efficiencies by quadrant are shown. The proton STOP efficiency is typically the worst scenario for the instrument, as protons produce the lowest yield of backward emitted secondary electrons (Ritzau and Baragiola 1998).

\subsection{Energy Measurement System}

Sixteen solid state detector (SSD) pixels are located at the end of the time-of-flight path in quadrants Q0 and Q1 of the TOF/E chamber. The SSDs provide two functions: (1) measurement of the residual energy (after pulse height defect) of the incident ion, and (2) emission of secondary electrons, which are used to trigger the stop signal for the coincident time of flight measurement.

The SSDs chosen for PLASTIC are custom made Canberra passivated implanted planar silicon (PIPS) detectors with an equivalent entrance window of $25 \mathrm{~nm}$ silicon. Eighteen SSD pixels are mounted on two macor silver-platinum metalized frames. Two of the pixels are obstructed by supporting structures and are not used in analysis. Table 5 provides the physical characteristics of the SSD pixels.

The energy measurement response function of silicon PIPS detectors includes the detector nonlinearity (pulse height defect as a function of incident particle species and velocity) and the shape and width of the response function (noise), including broadening effects from channeling (Collier et al. 1988; Oetliker 1993; Steinbauer et al. 1994). The silicon crystal $7^{\circ}$ off-axis orientation was specified to minimize channeling by normal incidence particles. Pulse height defect and noise response of the PLASTIC flight detectors were calibrated prelaunch at the MEFISTO beam facility (UBe). Measurements were made of ions $\mathrm{H}, \mathrm{C}, \mathrm{O}, \mathrm{N}$, and $\mathrm{Ar}$ in the energy range $10-600 \mathrm{keV}$. The calibration results are described in Blush et al. (2005).

The SSD Module for PLASTIC consists of the mounted solid state detectors and three electronic circuit boards. Two of the boards (SSD-A, SSD-B) are stacked directly behind the detector arrays, and are referenced to the PAC voltage. The remaining board (SSD-C) is located on the Signal Board, and is referenced to ground. Optical links using infrared emitter

Table 5 PLASTIC solid state detector characteristics

\begin{tabular}{ll}
\hline Manufacturer & Canberra Industries \\
Type & Custom PIPS \\
Pixel shape & Trapezoid \\
Pixel active area (nominal) & $95 \mathrm{~mm}^{2}$ \\
Number of pixels & $18(16 \mathrm{used})$ \\
Batch chip thickness, fully depleted & 502 microns \\
Batch resistivity & 13200 ohm-cm \\
Silicon wafer crystal orientation & $\langle 111\rangle 7^{\circ}$ off axis \\
Equivalent entrance window thickness & $25 \mathrm{~nm}$ \\
Operating voltage & $80-120 \mathrm{~V}$ (95 V in instrument) \\
FM1 serial numbers (quad 0,1) & $\mathrm{S} 8, \mathrm{~A} 5$ \\
FM2 serial numbers (quad 0,1) & $\mathrm{S} 3, \mathrm{~A} 1$ \\
\hline
\end{tabular}


FM2: Heavy Ions

Start Efficiency versus MCP

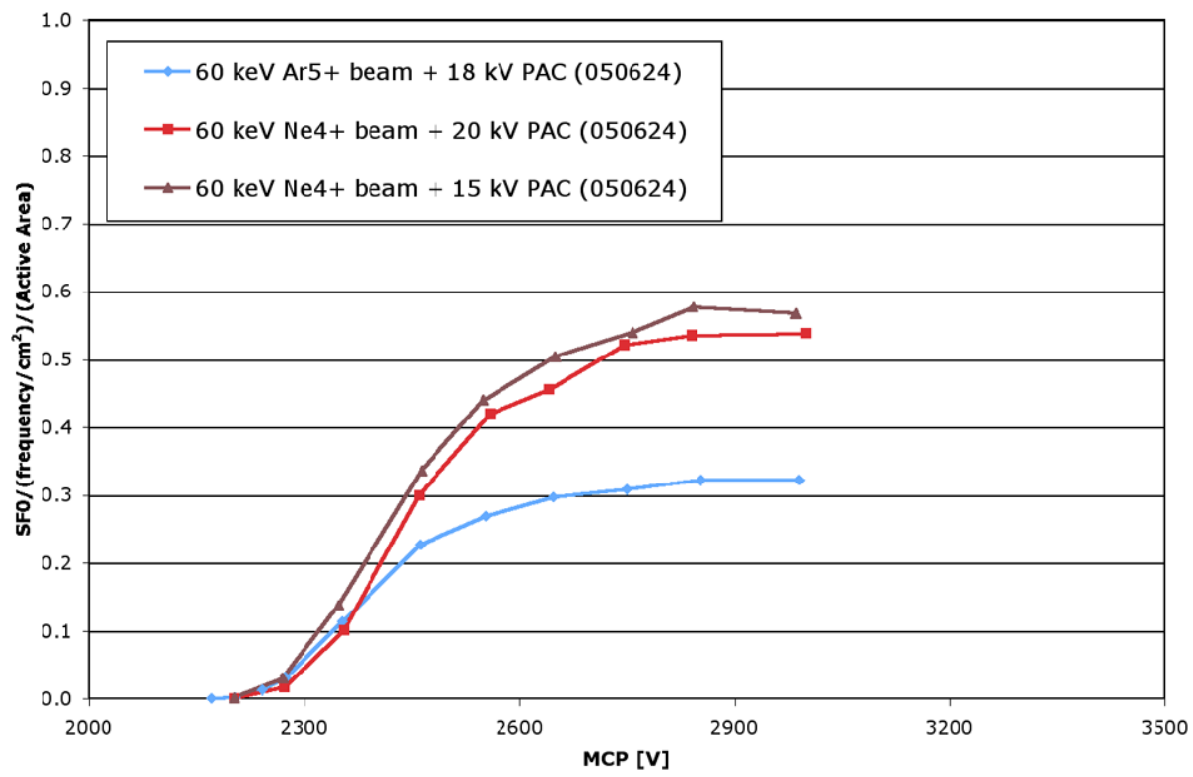

FM2: Heavy Ions

Stop Efficiency versus MCP

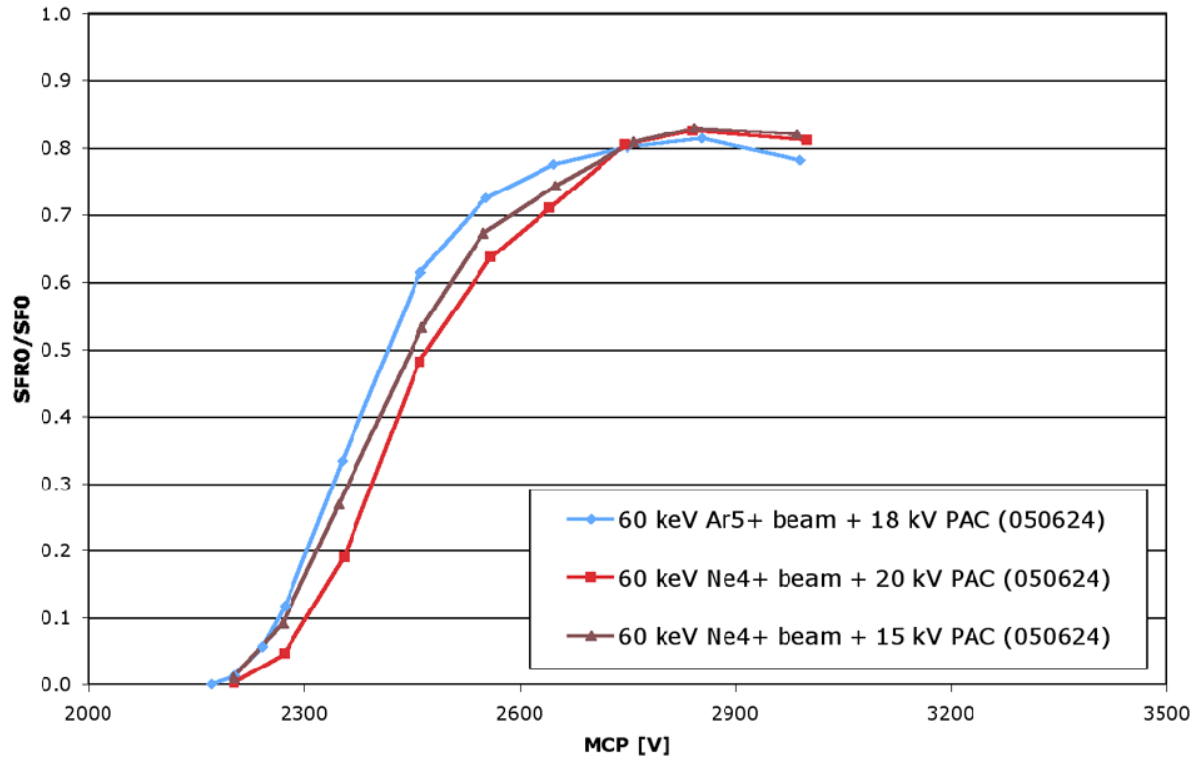

Fig. 9 (Top to bottom) START, STOP and VALID EVENT efficiencies for neon and argon in FM2/B, as a function of bias applied to the MCP detector system. (a) START efficiency is given by the start trigger ("start flag", SF) rate normalized to the calibration beam monitor flux (counts $\left./ \mathrm{s}-\mathrm{cm}^{2}\right)$ and illuminated area $\left(\mathrm{cm}^{2}\right)$. (b) The STOP efficiency is derived from the ratio of the TOF coincidence rate ("start flag reset", SFR) to the start trigger rate (SF). (c) The VALID EVENT required coincident TOF and Energy signals, and its efficiency is derived from the ratio of its respective rate to the start trigger (SF). Data was obtained at the UBe CASYMS beam facility 


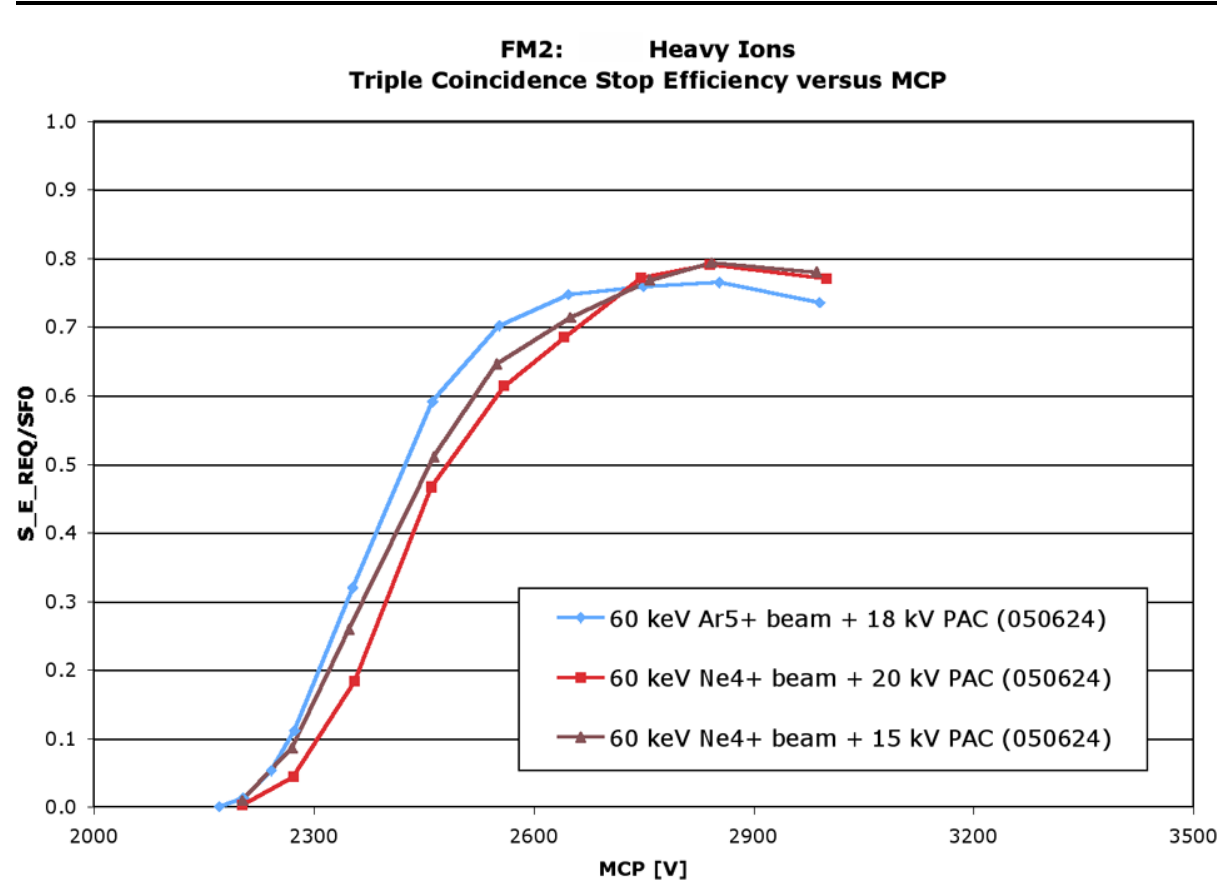

Fig. 9 (Continued)

Fig. 10 FM2/B STOP

efficiencies (given as the ratio SFR/SF) for $20 \mathrm{keV}$ total energy protons as a function of $\mathrm{MCP}$ bias, for quadrants Q0-Q3. Data was obtained at the UNH ion gun facility

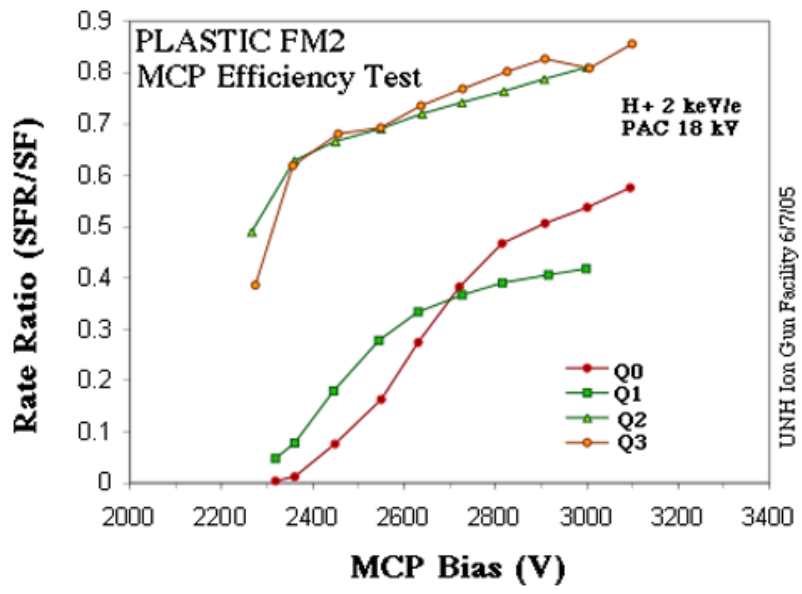

and diode detector pairs transfer signals across the PAC-to-Ground potential vacuum gap, that is, from the SSD-B to the SSD-C boards, and vice versa. These signals include uplinking of commands to an application-specific integrated circuit (ASIC) and downlinking of energy pulse height analysis (Energy PHA) values, rates, and housekeeping data.

The SSD-A board services the signals from the SSD pixels and includes an ASIC built by Ideas ASA (Norway). The Ideas XA16_HR was customized specifically for use with the PLASTIC SSD electronics and provides a high rate, 16-channel charge sensitive amplifier array, featuring low-noise buffered preamplifiers, shaping with peak and hold, individual 
channel threshold discriminators (commandable 8-bit DAC threshold settings), and internal calibration stimulation. Channels can be enabled/disabled individually in case a particular SSD pixel becomes noisy. The full-scale energy range is 15 to $2000 \mathrm{keV}$. Measured energies are converted to 10-bit (1024 bins) energy pulse height value with a nominal gain of $2 \mathrm{keV}$ per bin. The ASIC device was chosen over discrete electronics because of its low power consumption and small space requirements. As the PLASTIC ASIC represented new flight technology, the flight lot was tested for latch up susceptibility using heavy ion ( $\mathrm{Ar}, \mathrm{Cu}, \mathrm{Kr}$, Xe) beams at the Texas A\&M Single Event Effect Test Facility. Each flight ASIC's response at various temperatures was also calibrated within its respective SSD Module using the gamma and X-ray lines of radioactive cobalt $\left({ }^{57} \mathrm{Co}\right)$ and americium $\left({ }^{241} \mathrm{Am}\right)$ sources.

\subsection{Azimuth Position Measurement System}

The ESA and TOF/E optics are designed such that the original azimuth position information for the incident ion is maintained by the secondary electrons. Therefore, the entrance position of the ions can be determined from the position of the "START" pulse generated in the TOF System. PLASTIC measures the azimuth of incoming ions by using either discrete or continuous position detectors.

\subsubsection{Position by Discrete Anodes}

In quadrants Q1, Q2, and Q3, the azimuth is reported through ten discrete anodes. Each of these discrete anodes spans $22.5^{\circ}$ of azimuth and is plated directly onto the signal board. The charge accumulated on these anodes is detected by ten fast amplifiers and is reported if it exceeds threshold.

\subsubsection{Position by the Resistive Anode}

In quadrant Q0, which includes the solar wind sector, the azimuth is reported by a resistive anode (RA) position detector. The resistive anode offers better azimuth resolution than the discrete anodes. A photo of the signal board with the discrete and resistive anode position detectors is shown in Fig. 11.

The custom designed resistive anode is composed of a 25-micron thick resistive film layer deposited on an arc-shaped substrate with a mean radius of $45 \mathrm{~mm}$. The film is DuPont QS874, and the substrate is a $1 \mathrm{~mm}$ thick layer of $96 \%$ alumina. Contacts at each end are made of DuPont QS170 silver. The contacts were first bonded to the ceramic, and then the resistive film was deposited on top. The end-to-end resistance is approximately $50 \mathrm{kohms}$. The anode is placed on a $3.5 \mathrm{~mm}$ thick Ultem 1000 baseplate. This minimized the potential for damage from handling, and provided a durable connection point for a charge amplifier at each end.

A schematic of the resistive anode is shown in Fig. 12. The arc-shaped anode is shown with an MCP charge pulse deposited near the center. The deposited charge diffuses outward and is collected by charge amplifiers at each end of the anode. The location of the charge shower is calculated by collecting charge $Q_{\mathrm{A}}$ and $Q_{\mathrm{B}}$ from each end and entering this information into a lookup table held in EEPROM (Electrically Erasable Programmable Read-Only Memory) in the digital electronics. The axes of the table are values of $Q_{\mathrm{A}}$ and $Q_{\mathrm{B}}$. The content of the table is the distance from one end of the anode (A) to the point of charge deposition. The distances are calculated by:

Azimuth position $=Q_{\mathrm{B}} /\left(Q_{\mathrm{A}}+Q_{\mathrm{B}}\right) \times$ mean arc length. 
Fig. 11 A photo of the signal board is shown. The screens collect MCP showers from "start" electrons. The opaque panels collect MCP showers from "stop" electrons or ions. The black resistive anode can be seen below the solar wind sector screen in the foreground

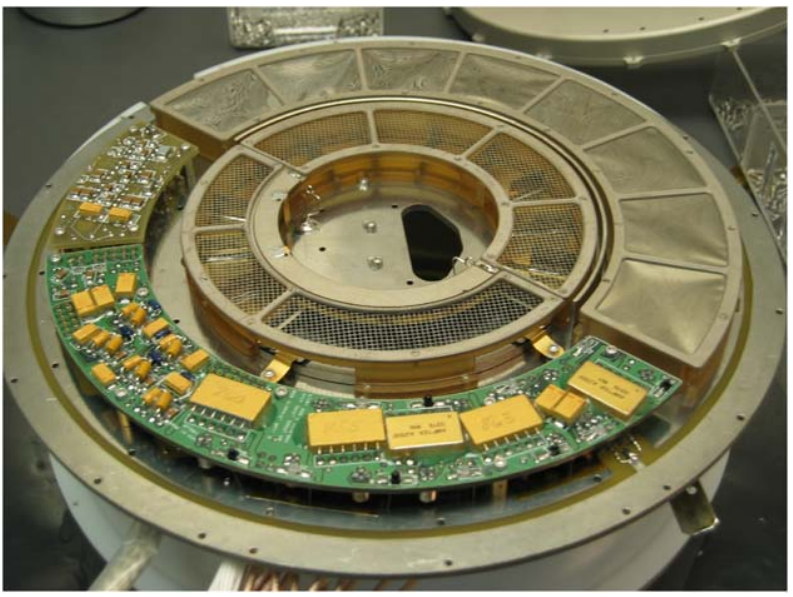

Fig. 12 A schematic of the resistive anode is shown. The location of the charge shower is calculated by collecting a charge $Q$ from each end and entering a lookup table. The contents of the table are distances from one end of the anode (A) to the point of charge deposition

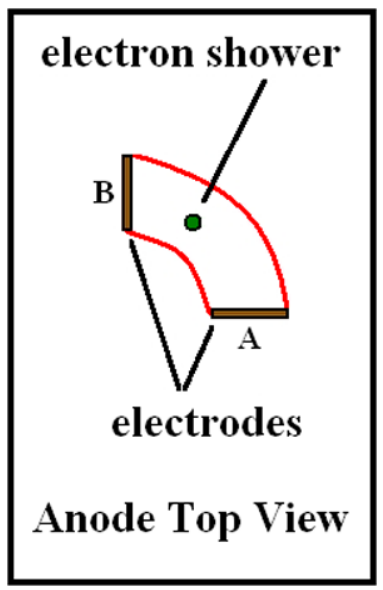

The use of a table simplifies and speeds up the position calculation. It also permits compensation for any gain imbalance between the two charge amplifiers.

The resistive anode position electronics include a charge amplifier for each end of the anode and a trigger for event counting, The trigger is set if the combined charge $Q_{\mathrm{A}}+Q_{\mathrm{B}}$ exceeds a threshold of $50 \mathrm{fC}$. The amplifiers have a gain of $5 \mathrm{mV} / \mathrm{fC}$.

An inherent challenge for the anode circuit is the accommodation of a wide range of MCP pulse heights. These pulses may reach $\sim 5 \mathrm{pC}$, depending on the MCP voltage. The resistive anode amplifiers may therefore saturate on $\mathrm{MCP}$ pulses from some ions. The circuit is designed to allow a delayed conversion, in which the position is acquired just after the amplifiers come out of saturation. The trigger pulse width is approximately $600 \mathrm{~ns}$ for nonsaturated cases, and approximately $2.8 \mu$ s for saturated pulses. A deadtime of approximately $2 \mu$ s has been derived from high rate tests. These results evidently include a mixture of saturated and non-saturated pulses. This design provides a fast counter with a wide dynamic range for high rate solar wind protons. 


\subsubsection{Resistive Anode Measurements}

The response of the resistive anode as a function of incoming beam angle is shown in Fig. 13. The mapping is unique over the central solar wind sector covering $\pm\left(3-19^{\circ}\right)$. The extreme position $\left(>20^{\circ}\right)$ response is relatively flat. This is in part because the anode is much longer than it is wide, and therefore the azimuth resolution is best near the center, where it is most critical for the science requirements. The sections beyond $\pm 20^{\circ}$ are located in the Suprathermal Wide Angle Partition (WAP) section, used for pickup and suprathermal ions. This section needs only $22.5^{\circ}$ azimuth resolution.

The position mapping in the center of the solar wind sector is double-valued in the $\pm 3^{\circ}$ range. This is caused by a structural support for the carbon foils. The support tends to electrostatically steer carbon foil knock-off electrons away from the center. In flight, the solar wind is expected to lie typically to one side of center, due to the aberration resulting from the speed of the spacecraft relative to that of the solar wind.

Resistive anode position distributions are shown in Fig. 14 for eight incoming ion beams used in pre-launch calibration. The beams entered the instrument azimuthally at angles between $+1^{\circ}$ and $-6^{\circ}$, measured from the center of the solar wind sector. The FWHM of the distributions in this particular example is $\sim 8^{\circ}$. The behavior of the position distribution for incoming azimuths of $3^{\circ}$ or less is shown. The distributions make a jump transition across the center of the solar wind sector in the $\pm 1^{\circ}$ range. Ions entering directly in the center of the solar wind sector are largely obstructed by carbon foil support structures.

At high rates, pulse pileup causes the deposited charge from several ions to be summed, in which case the circuit reports their average azimuth. This is acceptable in the solar wind, as only the bulk direction of the high flux solar wind protons is required.

In circumstances where a large quantity of charge is accumulated, the $\mathrm{A} / \mathrm{C}$ coupling of the amplifiers may broaden the position distribution. This may happen in cases where the rate is high, or MCP pulses are large for other reasons. This effect is observed, for example, when the MCP bias is high and heavy ions are detected. The broadening develops as the signal baseline drops below ground. A constant is therefore subtracted from the charge accumulated at each end of the anode. This tends to shift the calculated position away from the centroid.

Figure 15 shows the azimuth position reported for an increasingly high rate calibration beam of He ions from a single azimuth. The position peak channel decreases by approxi-

Fig. 13 An azimuth scan is shown in which the instrument is rotated past a fixed calibration beam. Plotted is the azimuth bin channel reported by the resistive anode vs. the incoming beam azimuth. The reported azimuth bin is uniquely related to the incoming azimuth from $\sim-19^{\circ}$ to $+19^{\circ}$. In the suprathermal sections beyond $\pm 29^{\circ}$, the anode reports a single value, corresponding to the $22.5^{\circ}$ resolution required for the WAP section

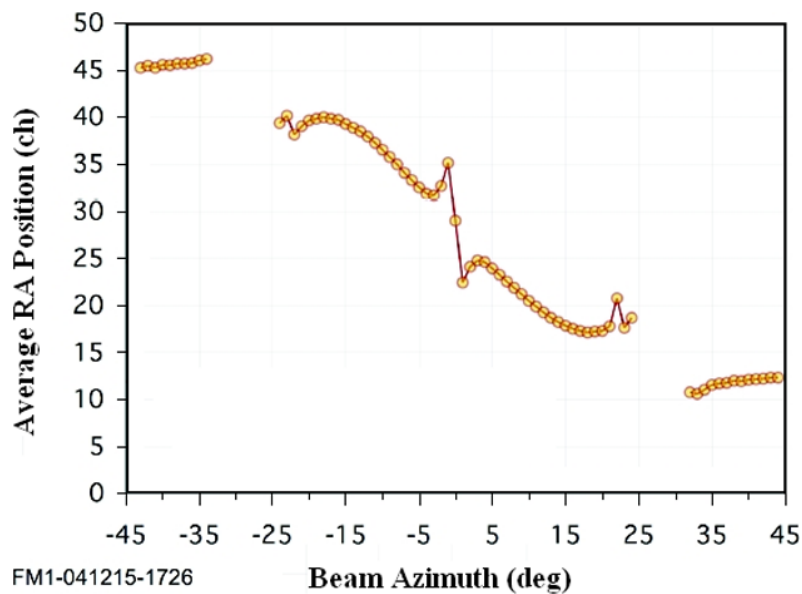


Fig. 14 The resistive anode output is shown for eight incoming ion beams during pre-launch calibration. Beam azimuths were +1 to -6 degrees from the center of the solar wind sector

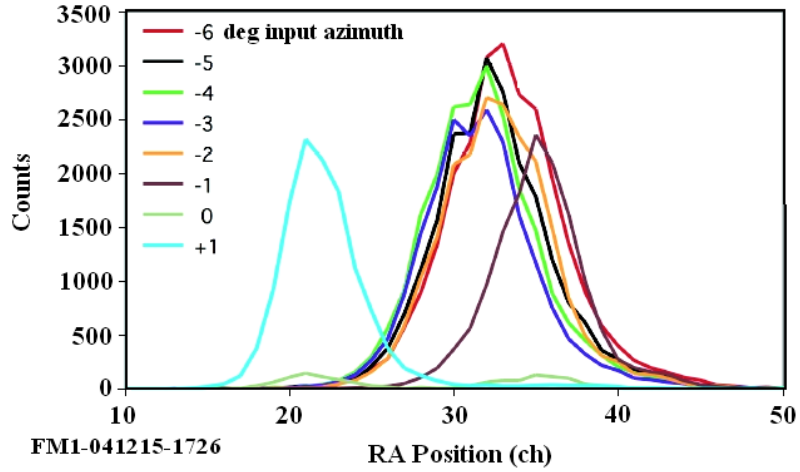

Fig. 15 The average and FWHM of the position reported by the RA is shown for a single calibration beam azimuth at increasing rates. Each RA channel corresponds to an angle bin of $\sim 1.3^{\circ}$ width

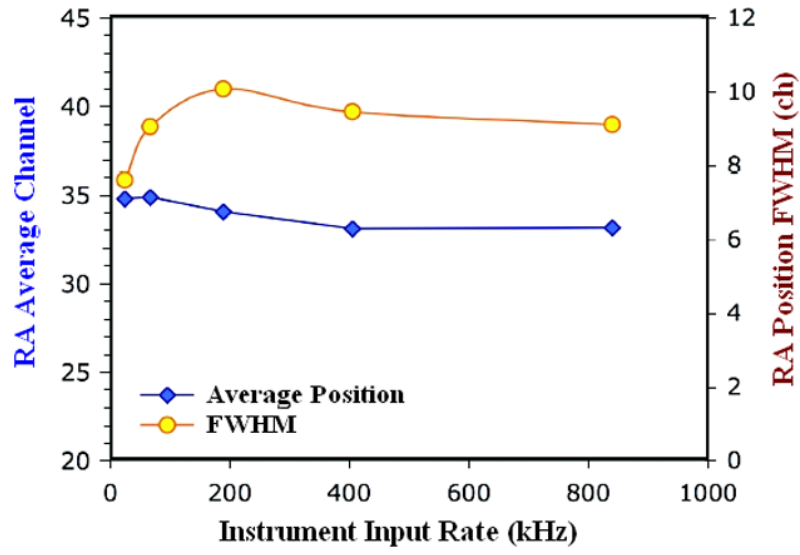

mately 2 channels as the rates increase (each channel represents a $1.3^{\circ} \mathrm{bin}$ ). The position distribution widens somewhat as well.

The uncertainty in the average azimuth depends on the number of ions collected in a single deflection step. For example, an input of $4 \times 10^{8}$ protons $/ \mathrm{s}-\mathrm{cm}^{2}$ to the Small-channel aperture would reduce to $\sim 600 \mathrm{kHz}$ on the resistive anode. The reported rate with dead time would be $\sim 100 \mathrm{kHz}$. A sampling duration of $12.8 \mathrm{msec}$ at each deflection step would then collect $\sim 1400$ events. The resulting uncertainty in the average azimuth would be $\sim 0.25^{\circ}$.

\subsection{Analog and Digital Electronics}

\subsubsection{Functional Overview}

The instrument electronics are contained on more than thirty circuit boards, with components that include seven Actel complementary metal-oxide-semiconductor (CMOS) field programmable gate arrays (FPGAs), five electrically erasable programmable read-only memories (EEPROMs), and an application-specific integrated circuit (ASIC) device. Some of the signal-processing electronics have been previously described under the corresponding measurement description section.

The functions of the sensor electronics include: 
- Providing the sensor interface to the IDPU for information transfer (commands and time ticks, data requests received from the IDPU; PHA events, rates and housekeeping data transmitted to the IDPU);

- Providing low voltage conversion and EMI filtering from the spacecraft $28 \mathrm{~V}$ power input;

- Controlling the sensor's operating mode;

- Controlling voltage sequences in the Entrance System, such as setting the sweeping voltages for the polar deflectors, enabling/disabling the Main Channel Gating, and stepping of the ESA high voltages;

- Providing, controlling, and distributing high voltages;

- Processing detector signals;

- Implementing logic selection criteria for the validation of events;

- Controlling information flow within the sensor, including the transfer of products forming the pulse height analysis (PHA) word;

- Building up the PHA word for each validated event and providing real time, high-speed, PHA event classification using table look-up techniques;

- Collecting Matrix Rates from the PHA classification, for transmission to IDPU;

- Collecting and selecting PHA events, for transmission to IDPU;

- Collecting Monitor Rates at predetermined intervals, for transmission to IDPU;

- Collecting sensor analog and digital housekeeping (HK), for transmission to IDPU;

- Providing internal stimulation for pre-flight and onboard functional tests; and

- Monitoring and responding to latch up conditions.

A simplified functional diagram for the analog and digital electronics is given in Fig. 16. Most of the circuits are referenced to ground and primarily reside either on the Signal Board (located in TOF Housing at ground) or else within the Electronics Box. Portions of the SSD Module and the MCP/SSD power supplies float relative to the PAC voltage (0 to -25000 V).

\subsubsection{Power Supplies (PS)}

There are a total of eight power supplies incorporated into the PLASTIC sensor:

- The low voltage power converter LVC (Luhmann et al. 2007);

- Four dynamic high voltage (HV) power supplies (together called the "SWEEP" supplies), which apply a pre-determined sequence of voltage settings (obtained from EEPROM tables) to the Entrance System's Main Channel polar deflectors (DEFL1, DEFL2, 0 to +6 $\mathrm{kV}$ ), with taps for the Small Channel polar deflectors; the two electrostatic analyzer voltages (ESA_plus, $+10 \mathrm{~V}$ to $+6 \mathrm{kV}$; ESA_minus, $-10 \mathrm{~V}$ to $-6 \mathrm{kV}$ ), and the Small-channel steerage (SCHN, 0 to $-4 \mathrm{kV}$ ), with taps for Small Channel tuning and for the Main Gate. A customized high-voltage, radiation-tolerant optocoupler was developed to control the voltage ramp for each of the SWEEPS. The ESA+/- cadence is 128 logarithmic steps in less than 60 seconds. The DEFL1 and DEFL2 power supplies are synchronously either held or ramped through appropriate voltages matched to a given set of ESA voltages;

- Three static power supplies incorporated into the TOF HV supply: the post acceleration PAC (0 to $-25 \mathrm{kV})$, the MCP detector voltage (0 to $4 \mathrm{kV})$, and the SSD detector and electronics bias $(\sim 100 \mathrm{~V})$. The MCP and SSD power supplies float at the PAC voltage.

\subsubsection{Onboard Classification and Data Handling (Sensor)}

The PLASTIC digital electronics generates three types of data information: (1) identification and signal pulse height information from each ion event ("Pulse Height Analysis Words" and 


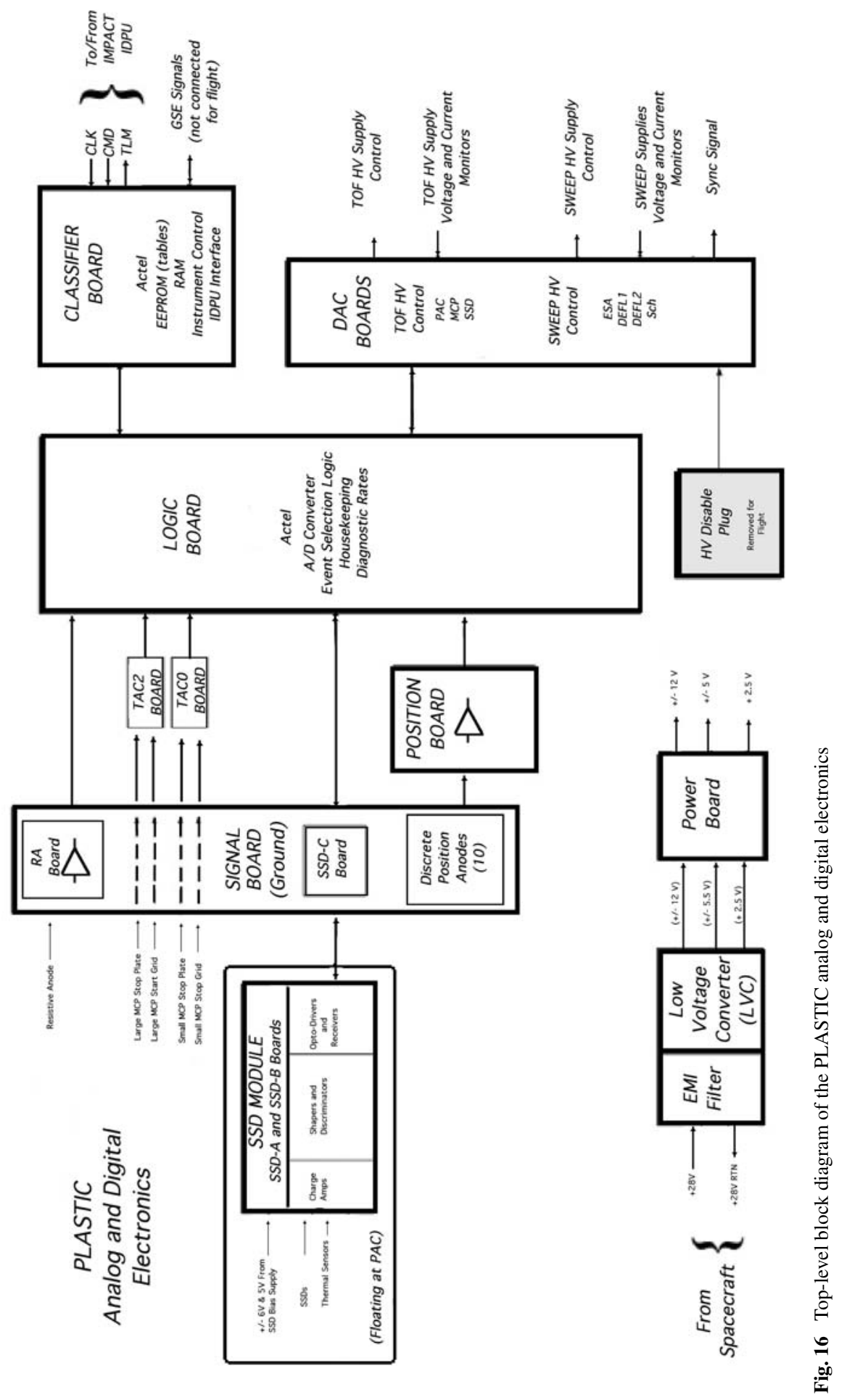


"Classified Matrix Rates"); (2) counts of various detector diagnostic "Monitor Rates" within specific time interval (each deflector step, $12.8 \mathrm{~ms}$ ); and (3) various instrument "Housekeeping and Status" information. Data types (2) and (3) are sent directly to the IDPU. The PHA Words are sent to the "classifier board", where they are further analyzed, as described below.

3.7.3.1 PHA Word An incident ion that satisfies the (commandable) event selection logic conditions is assigned a 48-bit pulse height analysis (PHA) word that contains the following information: The ESA voltage step (SWPE, 0-127) during which the event occurred; SWS polar deflection step (SWPD, 0-31); quadrant of triggered anode (QUAD, 0-3); measured energy in the SSD (SSDE, 10-bit channel number, $2 \mathrm{keV} / \mathrm{chn}$ ); identification of the SSD pixel triggered (SSD_ID, 0-15; or if no SSD was triggered, set to 0); time-of-flight (TOF, 10-bit channel number, $0.25 \mathrm{~ns} / \mathrm{chn}$ ); azimuth angle within given quadrant (POS, 64 bins in Q0; 2 bins in Q1; 4 bins each in Q2, Q3); section of instrument triggered (SWS-Main, SWSSchn, WAP-SSD, WAP-noSSD, SECTION assignment is $0-3$, respectively); and priority range assignment for PHA normalization (PRIORITY, 0-3). The priority determination is based on the event classification described next.

The digital electronics use the assigned priority to select a sample of 512 PHA events during each ESA step for transmission to the IMPACT/PLASTIC IDPU. These direct PHA events provide the most detailed and self-contained information possible for any event.

3.7.3.2 Event Species Classification Every PHA word is classified by mass and massper-charge through an onboard determination. Event classification by the digital electronics employs fast look-up table techniques that establish a direct correspondence between the SWPE, SSDE, TOF, and QUAD parameters provided in the PHA word to bin assignments from the Mass Tables $(\mathrm{Nm})$ and $M / Q$ Tables $(\mathrm{Nq})$ stored in EEPROM (see Fig. 17, and Table 6). The classification algorithm used to generate the look-up tables is based on the basic relationships presented previously under the principle-of-operation section. The algorithm incorporates the calibrated instrument response function for the energy loss in the carbon foil, the pulse height defect in the solid-state detectors, the TOF signal-to-channel conversions, and the measured energy signal to channel conversions. The algorithm depends on commandable instrument parameters, such as the voltage (hence energy-per-charge) assigned to a given SWPE value, and the PAC voltage value (0 to $-25000 \mathrm{~V})$. These look-up tables are not calculated onboard by the IDPU as done on previous missions (e.g., Gloeckler et al. 1992; Hovestadt et al. 1995), but are rather commanded as table loads directly into EEPROMS in the PLASTIC electronics. It is an important consequence to post-launch flight operations that the tables associated with species classification match any changes made to the ESA table assignments or to the commanded PAC voltage. Otherwise, an event may not be assigned to its appropriate Priority or Matrix Rate.

3.7.3.3 Matrixs Rates Data "Matrix Rates" correspond to the accumulated counts over a pre-defined accumulation time of selected ion species or groups of species (see Fig. 18). Matrix Rate "species box" definitions are based on assignments in a logarithmic Mass vs. $M / Q$ space as represented by the Nm, Nq binning as defined in Table 6.

The $\mathrm{Nm}, \mathrm{Nq}$ rate assignments are up-loaded into the EEPROM "Classification Bin Tables" by ground command, and thus can be reconfigured if desired during the mission.

"Priority Rates" are Matrix Rates that correspond to large scale (coarse species groupings) $M$ vs. $M / Q$ "species boxes" that are used in assigning a priority value to each PHA event. The priority rate accumulators are incremented for each and every PHA event whose calculated location in $(\mathrm{Nm}, \mathrm{Nq})$ space matches the assigned (Nm, Nq) priority bin table entry. The calculated priority value (0-3) is then inserted into each PHA word. Priority Rates 


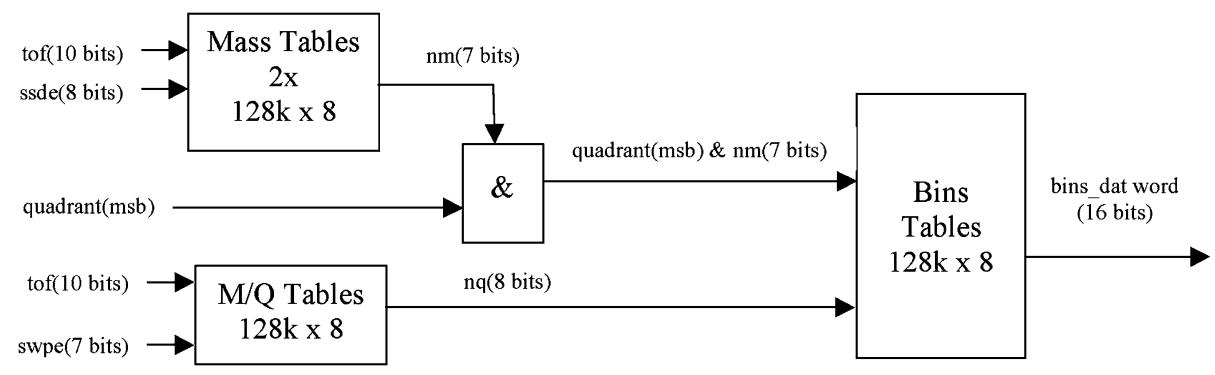

Fig. 17 Matrix Rate generation table look-up flow chart using table lookups from four $128 \mathrm{k} \times 8$ EEPROMs

Table 6 Mass and mass-per-charge binning

\begin{tabular}{ll}
\hline $\mathrm{Nm}$ & $\mathrm{Nq}$ \\
\hline Formula: & Formula: \\
Mass range $=M_{\min }$ to $M_{\max }$ & Mass/charge range $=M Q_{\min }$ to $M Q_{\max }$ \\
$N_{M}$ range $=1$ to $N M A X$ & $N_{Q}$ range $=1$ to $N Q M A X$ \\
$M$ (lower bound of $\left.N_{M}\right)=M_{\min } \cdot k_{M}^{\left(N_{M}-1\right)}$ & $M / Q\left(\right.$ lower bound of $\left.N_{Q}\right)=M Q_{\min } \cdot k_{Q}^{\left(N_{Q}-1\right)}$ \\
$k_{M}=\left(M_{\max } / M_{\min }\right)^{1 / N M A X}$ & $k_{Q}=\left(M Q_{\max } / M Q_{\min }\right)^{1 / N Q M A X}$ \\
& \\
Parameters at launch: & Parameters at launch: \\
$M_{\min }=0.5$ amu & $M Q \min =0.5$ amu/e \\
$M_{\max }=95.0$ amu & $M Q \max =60.0 \mathrm{amu} / \mathrm{e}$ \\
$N M A X=90$ & $N Q M A X=254$ \\
$k_{M}=1.06$ & $k_{Q}=1.02$ \\
\hline
\end{tabular}

are used for the normalization of the PHA words for ground analysis of flux determinations. There are four classification priorities for the Solar Wind Sector and two priorities for the Suprathermal WAP Sectors. The solar wind priorities are illustrated in Fig. 18 as blue outlined boundaries in $M$ vs. $M / Q$ matrix space.

There are a total of 47 Matrix (including Priority) Rates divided between the SWS and Suprathermal WAP Sectors. Some of these retain full polar, azimuth, and ESA stepping information $(32 \times 32 \times 128)$ as they are transmitted to the IDPU. Others are summed, resulting in coarser directional resolution. The "Matrix" Rates products, constituting 13 Kbytes, are sent to the IDPU every ESA step, where they are further processed to create the final data products that fit into the PLASTIC telemetry allocation of $3 \mathrm{kbps}$.

\subsection{Spacecraft Accommodation}

The STEREO observatories are three-axis stabilized and sunward-pointing. The PLASTIC$A$ and PLASTIC-B instruments are identically mounted on the sunward-facing $+X$ panels of the STEREO-A and -B spacecraft, overhanging the respective spacecraft $+Y$ panels (Fig. 19). There are minor differences in the two spacecraft mountings in the thermal control features and in the extent and location of external obstructions in the FOVs. Instrument physical resource requirements are provided in Table 7.

The FOV for the solar wind sector is $\pm 20^{\circ}$ in the polar (nominally centered on the ecliptic plane) direction, and is sun-centered $45^{\circ}$ in the azimuth (in-ecliptic) direction for both 


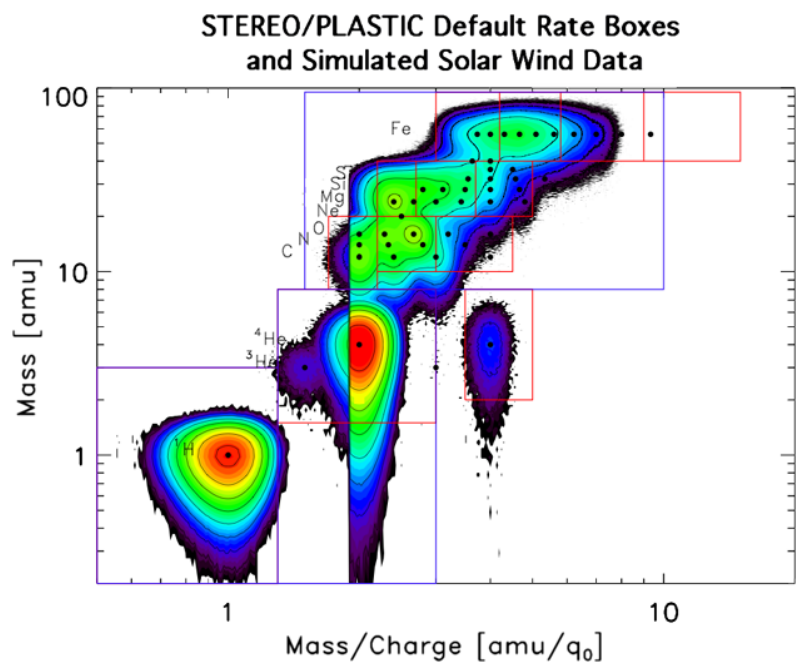

Fig. 18 Default Matrix (red) and Priority (blue) rate "boxes" in logarithmic Mass and $M / Q$ space as defined for the Solar Wind Sector. Rate boundary definitions can be changed by command, and need not consist of contiguous boxes. Superimposed on the $M$ vs. $M / Q$ space are contour plots of a simulated ion population corresponding to a coronal charge state temperature of 1.7 MK (Mazzotta et al. 1998) and nominal solar wind-like abundances. The singly charged helium illustrates a different source population, such as pick up ions. The "ridge" seen just above $M / Q>2$ and the suppressed counts at $\mathrm{He}^{+2}, \mathrm{C}^{+6}$ and $\mathrm{H}^{+}$illustrate the simulated switching in the Solar Wind Sector entrance from the Main Channel geometrical factor to the Small Channel geometrical factor

the Small and Main Channels. The FOVs in the solar wind sector are unobstructed. For the suprathermal ions wide-angle-partition (WAP) sector, a polar FOV of $\pm 7^{\circ}$ and azimuth FOV of $305^{\circ}$ were originally requested. However, practical accommodations with spacecraft structures (solar panel arrays, SA tie downs and snubbers, the spacecraft bus and thermal blanket) and with the required location (for facing the Parker spiral direction) for the IMPACT SEP packages created impingements in both FOV directions (Fig. 20). These blocked areas were in some instances utilized within the instrument as the locations for internal structural supports.

Each PLASTIC sensor is thermally isolated from the respective spacecraft deck through the use of six mounting feet isolators (which also served to dampen vibration at launch). Thermal blankets, radiator surfaces, and two survival heater circuits are used for thermal control. The survival heaters are controlled by mechanical thermostats and by spacecraft monitoring. One heater $(4 \mathrm{~W})$ is located on the starboard radiator of the Electronics Box. Two $2 \mathrm{~W}$ heaters are placed on the TOF/E Chamber Housing. The multi-layer insulation (MLI) thermal blanket material on STEREO is made of perforated ITO Silver Teflon. This material meets the spacecraft electrostatic cleanliness requirements. On STEREO-A, which has the higher solar heat input as a result of its closer heliocentric orbit, an exterior auxiliary radiator that is mounted on the $+Y$ deck provides additional heat sinking for PLASTIC. The primary thermal concern is the regulation of the micro-channel plates (MCP) and solid state detectors (SSD) to temperature ranges of $-25^{\circ} \mathrm{C}$ to $+25^{\circ} \mathrm{C}$ and $-20^{\circ} \mathrm{C}$ to $35^{\circ} \mathrm{C}$, respectively. Pre-launch thermal design incorporated radiation models created in Thermal Synthesizer System (TSS) and temperature analysis using SINDA, and then predictions were compared with instrument and spacecraft thermal vacuum. 


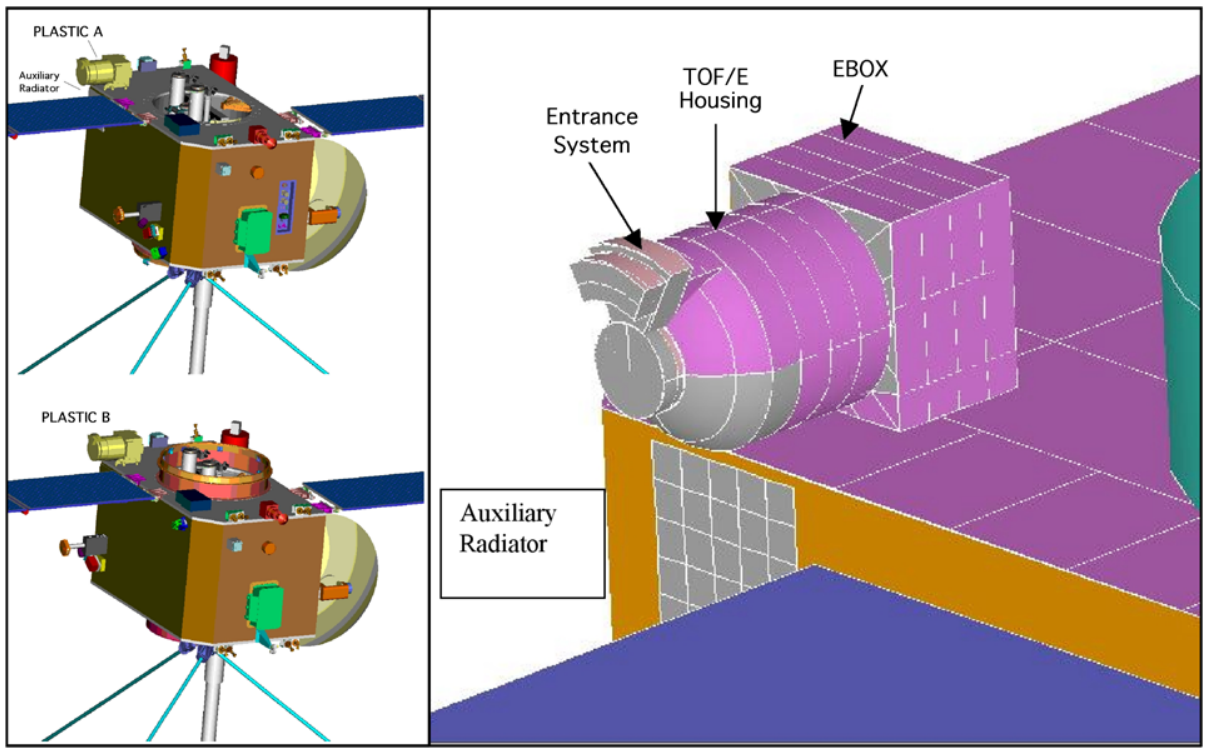

Fig. 19 (a) The mounting location of the PLASTIC sensor on the top deck (sunward facing) of the STEREO-A (top) and STEREO-B (bottom) spacecraft. (b) Schematic view of the PLASTIC sensor. The corner overhang on the spacecraft deck provides the field of view for the WAP sector. Auxiliary radiator is on the A spacecraft, only

\section{Instrument Data Products}

The IDPU receives from the sensor interface the classifier data (Matrix Rate count accumulators, prioritized PHA), data from the Monitor Rate accumulators, Housekeeping data, and instrument status. The DPU formats the various data products into a data stream that fits into the telemetry allocation of 3 kilobits/sec.

Housekeeping products are sampled once per minute during normal (science) operations. There are in excess of 300 housekeeping and status products, including those status products relating to IDPU operations.

Monitor Rate data consist of raw counts from individual 16-bit counters corresponding to 32 diagnostic rates. These are sent to the IDPU every $12.8 \mathrm{msec}$ (every deflection bin).

Matrix Rate data consists of raw counts from individual 16-bit counters that correspond to 47 selected species boxes. These are sent to the IDPU every $435.6 \mathrm{msec}$ (every ESA step). Prioritized PHA events are also sent to the IDPU every ESA step.

The IDPU compresses the accumulator counts from a 16-bit to an 8-bit representation, and performs sums on various Matrix and Monitor rates in terms of angle bins, energy bins, and/or time resolution, in order to further reduce the required telemetry rate. In addition, the IDPU down-selects the prioritized PHA data. The IDPU PHA down-selection is designed such that the final transmitted selection covers the full range of energies, but emphasizes the heavy ion data.

The data-related tasks performed by the IDPU include creating moments from the solar wind proton distribution, finding the peaks in the solar wind proton and alpha distributions, and creating reduced data products that contain the distribution surrounding these peaks.

The PLASTIC data transmission utilizes three possible telemetry modes: Science Mode $(\mathrm{S})$, Proton Mode $(\mathrm{P})$, and Engineering Mode (E). Only one telemetry mode is enabled by 
Table 7 PLASTIC spacecraft resources

\begin{tabular}{|c|c|c|}
\hline Resource & Value & Comments \\
\hline Size $(L \times W \times H)$ & $\begin{array}{l}479 \times 243 \times 265 \mathrm{~mm}^{3} \\
202 \times 202 \times 1.5 \mathrm{~mm}^{3}\end{array}$ & $\begin{array}{l}\text { Sensor only } \\
\text { Exterior Auxiliary Radiator (STEREO-A) }\end{array}$ \\
\hline Mass & $11.4 \mathrm{~kg}$ & $\begin{array}{l}\text { Sensor and mounting feet, does not include } \\
\text { external thermal components (blanket, ext. } \\
\text { radiator) nor IDPU harness }\end{array}$ \\
\hline Power & $\begin{array}{l}12 \mathrm{~W} \\
8 \mathrm{~W}\end{array}$ & $\begin{array}{l}\text { Sensor, } 28 \mathrm{v} \\
\text { Survival Heaters, } 100 \% \text { duty cycle }\end{array}$ \\
\hline \multicolumn{3}{|l|}{ Telemetry } \\
\hline Assigned APID & 200 & IMPACT/PLASTIC Combined HK \\
\hline Ranges (hex) & $\begin{array}{l}300-36 \mathrm{~F} \\
370-37 \mathrm{~F}\end{array}$ & $\begin{array}{l}\text { PLASTIC Data (Science, Engineering) } \\
\text { PLASTIC Beacon }\end{array}$ \\
\hline Average Rate & $3.2 \mathrm{kbps}$ & Nominal Real Time Allocation \\
\hline \multicolumn{3}{|l|}{ Field of view } \\
\hline $\begin{array}{l}\text { SWS Proton } \\
\text { Sweeping } \\
\text { Instantaneous }\end{array}$ & $\begin{array}{l}45^{\circ} \times 40^{\circ}(E / Q<10 \mathrm{keV} / \mathrm{e}) \\
45^{\circ} \times 0.3^{\circ}(\mathrm{FWHM})\end{array}$ & $\begin{array}{l}\text { SWS is Sunward centered in azimuth, polar } \\
\text { centered on ecliptic plane }\end{array}$ \\
\hline $\begin{array}{l}\text { SWS Main } \\
\text { Sweeping } \\
\text { Instantaneous }\end{array}$ & $\begin{array}{l}45^{\circ} \times 40^{\circ}(E / Q<40 \mathrm{keV} / \mathrm{e}) \\
45^{\circ} \times 2^{\circ}(\mathrm{FWHM})\end{array}$ & \\
\hline $\begin{array}{l}\text { WAP-STEREO-A } \\
\text { WAP-STEREO-B }\end{array}$ & $\begin{array}{l}50 / 175^{\circ} \times 3^{\circ}(\text { FWHM }) \\
50 / 160^{\circ} \times 3^{\circ}(\text { FWHM })\end{array}$ & $\begin{array}{l}\text { WAP is non-sunward in azimuth, polar centered on } \\
\text { ecliptic plane. Azimuth shown as SSD/noSSD }\end{array}$ \\
\hline
\end{tabular}

the IDPU at a given time. This mode is selected by ground command. Level 0 data products for all PLASTIC modes are listed in Table 8.

In Science Mode (normal science operations), the science products in the solar wind sector (SWS) consist of on-board computed moments for the solar wind protons, distributions of the solar wind protons and alphas surrounding the peak energy and angle, PHA events, and a number of Matrix Rates covering a variety of the heavy ion species. (Figure 18 illustrates a mass $v s$. mass-per-charge PHA data representation with the default Matrix Rate "species boxes" for the SWS.) The WAP science products also include PHA events and Matrix Rates, including proton, alpha, and heavy ion rates. All science rate definitions are commandable, and may change after launch.

In Proton Mode (P), the entire energy vs. angle array for solar wind protons is included in the telemetry. The primary purpose of this mode is for occasional in-flight verification of the proton moment determinations. In order to make room for this data product in the telemetry, there are no heavy ion Matrix Rates included, only the Priority Rates. In this mode, the heavy ion analysis can only be done using the PHA data. 


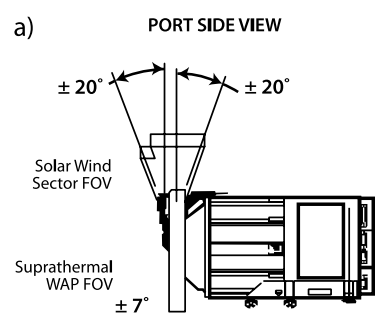

b)

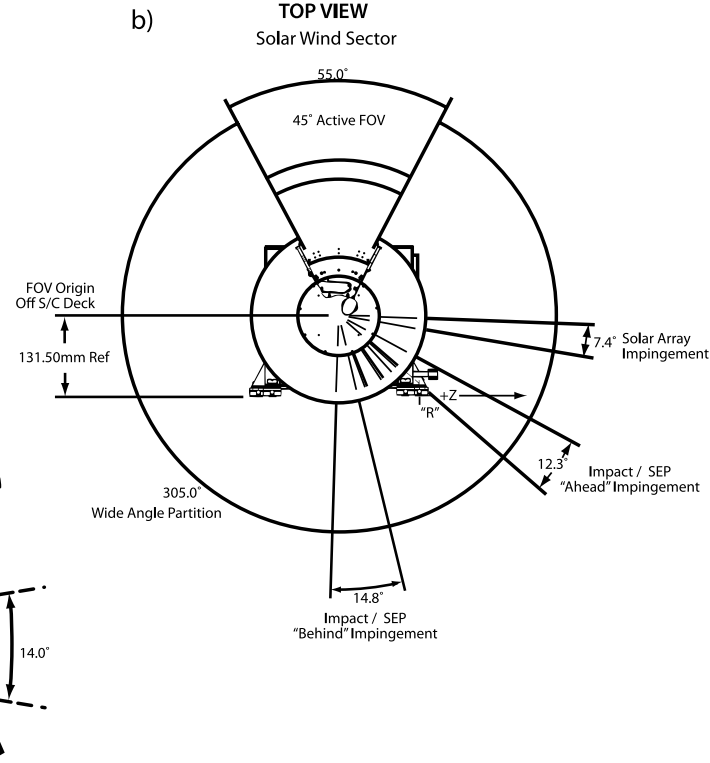

PLASTIC FOV OBSTRUCTIONS (POLAR DIRECTION)

Fig. 20 (a) Port side view of the instrument, indicatiing the polar FOV for the SWS and the WAP. (b) Top view of the azimuth (in-ecliptic) FOV, indicating locations of azimuth impingements for the mounted instrument. (c) Schematic front view of instrument mounted on the spacecraft, indicating polar impringements to the FOV

In Engineering Mode (E), the diagnostic Monitor Rates are transmitted with full time resolution. There are no science products in this mode, and it is primarily meant for commissioning and trouble-shooting.

In addition to the normal science products, the STEREO mission allocates a small amount of realtime telemetry to Beacon Mode (B) data products. These data products are provided for the purpose of space weather forecasting (Biesecker et al. 2007). For PLASTIC, these products include the solar wind proton density, bulk speed, kinetic temperature, and representative composition information.

\section{First Light}

STEREO was successfully launched on October 26, 2006, and PLASTIC commissioning began in late November. We report here some initial observations, taken during the commissioning phase, which illustrate the three science functions of the instrument.

\subsection{Solar Wind Proton Distributions}

Shown in Fig. 21 is a 7-day time-series stack plot of the intensities of solar wind protons observed on STEREO A. The horizontal axis corresponds to $E / Q$, from which the bulk speed is derived. Intensity is represented by the height of the distribution. During this sevenday period, there is a transition from slow to fast solar wind, with a weak CIR-associated forward shock. The fast solar wind originates from the central meridian passage of a coronal hole. 
Table 8 PLASTIC Level 0 Data

\begin{tabular}{|c|c|c|}
\hline Apid & Data product & Mode availability \\
\hline 200 & Analog Housekeeping (also includes IMPACT data) & $\mathrm{S}, \mathrm{E}, \mathrm{P}$ \\
\hline 313 & Digital Housekeeping & $\mathrm{S}, \mathrm{E}, \mathrm{P}$ \\
\hline 315 & PHA Data & $\mathrm{S}, \mathrm{P}$ \\
\hline 316 & Normal Monitor Rates & $\mathrm{S}, \mathrm{E}$ \\
\hline 317 & Full Resolution Monitor Rates & $\mathrm{S}, \mathrm{E}$ \\
\hline 318 & 'Trickle-Down’ Memory Dump & $\mathrm{S}$ \\
\hline 319 & Meta-data for Heavy Ions (number of packets summed) & $\mathrm{S}$ \\
\hline $31 \mathrm{~A}$ & SW $Z>2$ - High: Class 0 & $\mathrm{~S}$ \\
\hline $31 \mathrm{~B}$ & SW $Z>2$ - High: Class 1 & $\mathrm{~S}$ \\
\hline $31 \mathrm{C}$ & SW $Z>2$ - Low: Classes $2-9$ & $\mathrm{~S}$ \\
\hline $31 \mathrm{D}$ & SW $Z>2$ - Low: Classes 10-14 & $\mathrm{S}$ \\
\hline $31 \mathrm{E}$ & WAP-SSD-TCR & $\mathrm{S}$ \\
\hline $31 \mathrm{~F}$ & WAP-SSD-DCR & $\mathrm{S}$ \\
\hline 320 & WAP-noSSD-DCR & $\mathrm{S}$ \\
\hline 321 & SW Priority Rates & $\mathrm{S}, \mathrm{P}$ \\
\hline 322 & WAP Priority SSD & $\mathrm{S}, \mathrm{P}$ \\
\hline 323 & WAP Priority no-SSD & $\mathrm{S}, \mathrm{P}$ \\
\hline 324 & SW-All H-Alpha Reduced Distribution & $\mathrm{S}$ \\
\hline 325 & SW-H (Doubles) H+Peak Reduced Distribution \& Proton Moments & $\mathrm{S}, \mathrm{P}$ \\
\hline 326 & SW-Alpha (Doubles) $\mathrm{He}^{++}$Peak Reduced Distribution & $\mathrm{S}$ \\
\hline 327 & SW-Alpha (Triples) $\mathrm{He}^{++}$TCR Reduced Distribution & $\mathrm{S}, \mathrm{P}$ \\
\hline 328 & Compressed Classifier Data (Proton Mode) & $\mathrm{P}$ \\
\hline 329 & Uncompressed Classifier Data (Proton Mode) & $\mathrm{P}$ \\
\hline 370 & Beacon Data & B \\
\hline
\end{tabular}

Each horizontal trace represents a single sweep in $E / Q$ through the solar wind velocity range. There are 1440 sweeps per day (cadence 1 minute). Seen to the right of the stack plot are selected one-minute snapshots of the full resolution rate. The protons were measured in the Small Channel of the Solar Wind Sector, the alphas and trace elements in the Main Channel.

\subsection{Solar Wind Minor Species}

Figure 22 illustrates the switching from the Main Channel (large geometrical factor) to the Small Channel (small geometrical factor) that allows the measurement of both minor species and the more abundant protons and alphas with one common ESA system. At the lower ESA Step (higher $E / Q$ ) values, the Solar Wind Sector is open in the Main channel, providing excellent statistics for minor species, such as pickup helium, solar wind iron and oxygen. In this selected time interval, the IDPU onboard control ordered the Main channel closed (electrostatically) at about ESA step number 76, and the Small channel was enabled. To the right of this ESA step, the suppressed solar wind helium and some oxygen can still be seen in the Energy determination. The protons are seen at about ESA step number 96 in the top figure. Only a small percentage of the protons have sufficient energy to trip the SSD threshold, but they are readily observed in the TOF pulse height. 

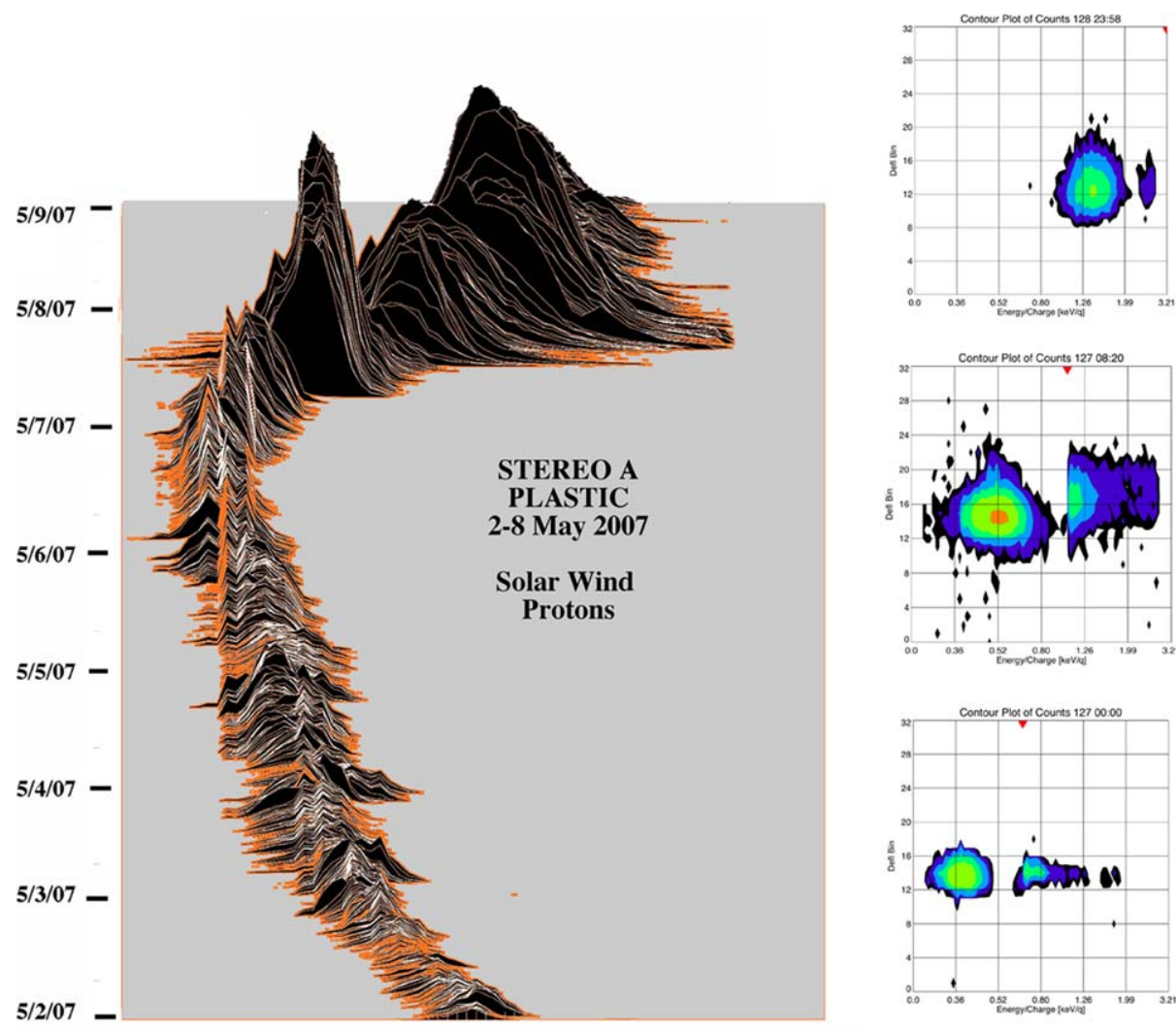

\section{Energy per Charge}

Fig. 21 Left: Seven-day time series stack plot of solar wind proton distributions observed by the Small Channel of the solar wind sector, summed over polar and azimuth directions (PLASTIC on STEREO A). The distributions can be used to yield proton bulk parameters, such as speed, density and kinetic temperature. Right (bottom to top): One-minute snapshots of the full resolution monitor rate for a slow, shocked, and high-speed solar wind during this time period. Data shows intensity (color contour) plotted against $E / Q$ (horizontal-axis) and polar deflection (vertical axis). A red arrow at the top of each panel denotes the switch between the Main (minor ions) and Small (proton) Channels

Species tracks in energy versus TOF in the Solar Wind Sector Main Channel are shown in Fig. 23 for PLASTIC B. Each element has its own track in E-T space. An ion's position on its track depends on the incident energy and the PAC value. Higher energy particles of a given species move up in measured energy and to the left in time-of-flight. If the $E / Q$ information from the ESA step number is included, individual charge states can be distinguished. The helium track shown here contains both $\mathrm{He}^{+2}$ and $\mathrm{He}^{+}$.

\subsection{Suprathermal Ion Populations}

One of the suprathermal heliospheric ion populations of interest for STEREO is interstellar pickup ions and their accelerated component at interplanetary shocks. The $M / Q$ histogram in Fig. 24a shows helium observed by PLASTIC-A over a two-hour interval during a highspeed ( $\sim 620 \mathrm{~km} / \mathrm{s}$, or $\sim 2 \mathrm{keV} / \mathrm{nuc}$ ) solar wind stream. Pickup helium $\left(\mathrm{He}^{+}\right)$is a prominent 
Fig. 22 Two hours of accumulated pulse height data (PHA) events taken from PLASTIC A. Data are not normalized by priority. (Top) PHA event frequency against ESA Step. All PHA have at least a TOF signature, but an Energy measurement is not required. (Bottom) Contour of Energy measurements against ESA step for the same time period
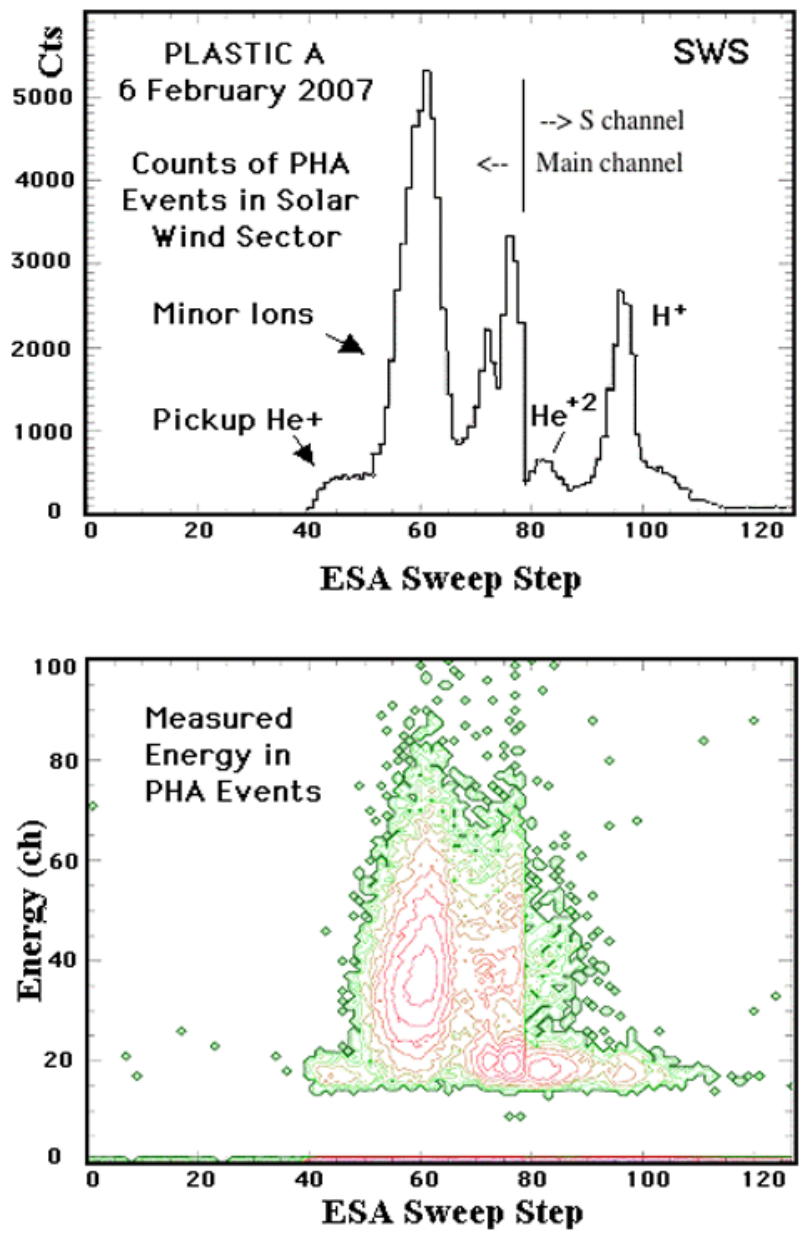

constituent during this period, and is easily distinguished from solar wind $\mathrm{He}^{+2}$. When the frequency of the identified $\mathrm{He}^{+}$is plotted against the incident $E / Q$ (Fig. 24b), the characteristic pickup ion cutoff energy is easily observed. (In the spacecraft frame, the cutoff energy is approximately four times the solar wind energy; in this instance corresponding to $\sim 8 \mathrm{keV} /$ nuc, or $\sim 32 \mathrm{keV} / \mathrm{e}$ for $\mathrm{He}^{+}$).

The source of the helium pickup ions are likely interstellar neutral atoms that pass through the heliosphere, become ionized, and are subsequently "picked up" and accelerated by the interplanetary magnetic field (a process first directly observed by Möbius et al. 1985 and described therein). The interstellar neutrals are gravitationally focused into a conical region on the opposing side of the sun from the direction of entry as the heliosphere travels through the local interstellar medium. The Earth passes through this focus cone in December of each year. One of the science opportunities for STEREO, as the observatories move away from Earth, will be able to provide direct in situ measurements of the focusing cone at alternative times of the year, for direct comparison with near-Earth (ACE, IBEX) or out-of-ecliptic (Ulysses) measurements. 


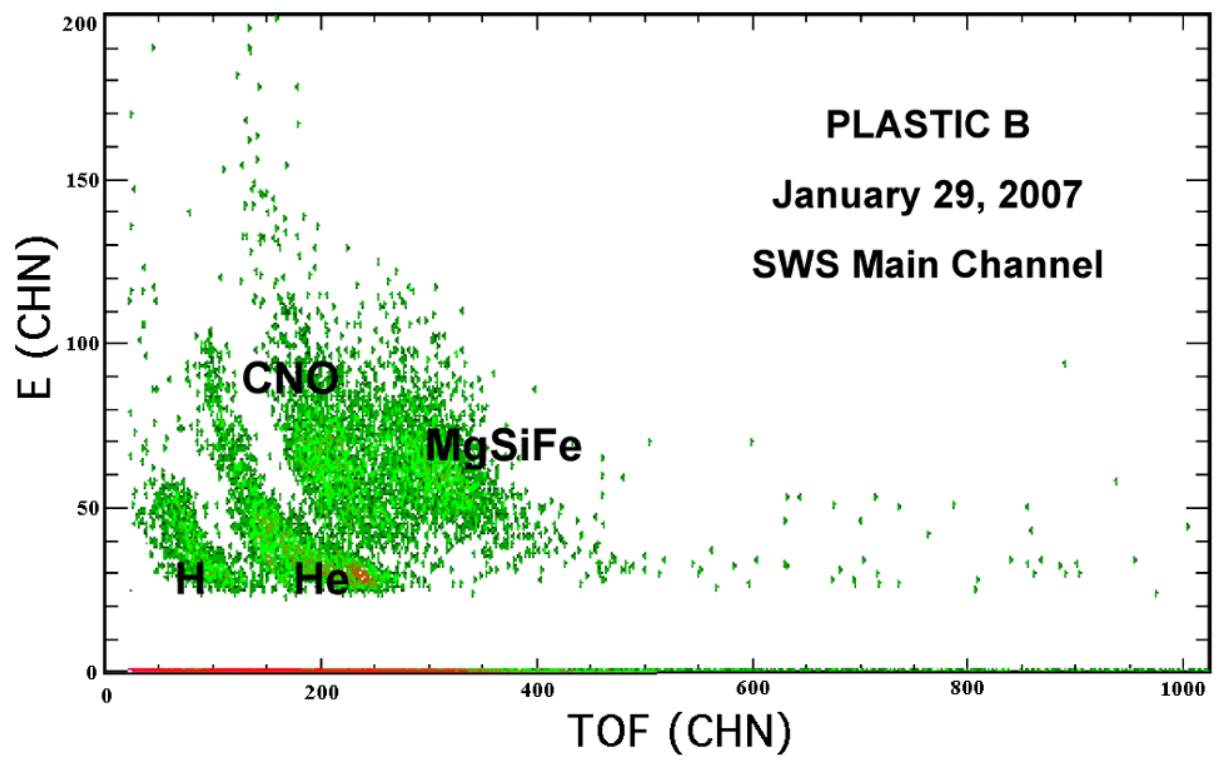

Fig. 23 One hour of composition data taken from the Main Channel on PLASTIC B. Data illustrates the species separation in mass resolution
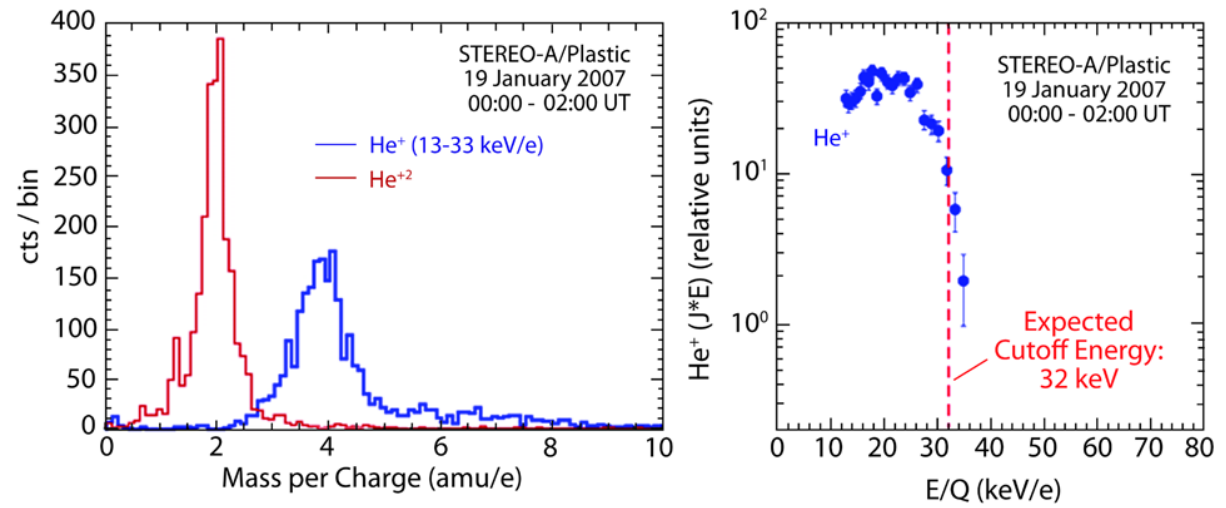

Fig. 24 (a) Mass per charge spectrum for helium, showing separation of the two charge states. (b) Two-hour accumulation count spectrum for pickup $\mathrm{He}^{+}$, showing the expected cutoff at twice the solar wind speed (four times the solar wind energy)

These and other initial measurements by the STEREO observatories indicate that the PLASTIC instruments are meeting all functional requirements to address the science objectives.

Acknowledgements The PLASTIC investigation is an international (USA, Switzerland, Germany) effort involving the University of New Hampshire, the University of Bern, the Max-Planck-Institute for extraterrestrial Physics, Christian-Albrecht-University Kiel, NASA/Goddard Space Flight Center, and the University of California, Berkeley (for the shared IMPACT IDPU and LVC). The specific contributions and responsibilities from these institutions are detailed in Table 9. 
Table 9 PLASTIC institutional contributors

Institution Contributions

University of New Hampshire (UNH)

University of Bern (UBe)

Max Planck Institute for extraterrestrial Physics (MPE)

Christian-AlbrechtUniversity Kiel (CAU)

NASA Goddard Space Flight Center (GSFC)

University of California, Berkeley (UCB)
PLASTIC Principal Investigator (PI) institution. Overall PLASTIC management (except for LVC/IDPU). Time-of-Flight/Energy Chamber and Housing (ion optics), Electronics Box, Digital Electronics, Solid State Detector (SSD) Frames and SSD Module Electronics, High Voltage Power Supplies, Position Anodes and Signal Electronics, Auxiliary Radiator, Damper modifications, Thermal, Electronic Ground Support Equipment (EGSE), Mechanical Ground Support Equipment (MGSE), Data System, PLASTIC Operations Center (POC) and Flight Operations, Data Analysis, and Education/Public Outreach (EPO)

PLASTIC Co-Investigator (Co-I) institution. Entrance System (Energy/charge Analyzer), Solid State Detectors (SSD), Microchannel Plates (MCP), SSD \& MCP Detector Testing, UV and Ion Beam Calibration Facilities and Support, Data Analysis.

PLASTIC Co-Investigator (Co-I) institution. Time-Amplitude-Converter (TAC) Electronics, Carbon Foils, Dampers, design for Position (POS) Board, Test Support, Data Analysis.

PLASTIC Co-Investigator (Co-I) institution. Electronics Box Panels, MGSE, Test Support, Data Analysis.

PLASTIC Co-Investigator (Co-I) institution. Calibration and Test Facilities, Test Support, Data Analysis.

IMPACT PI institution. Manages and provides shared IMPACT/PLASTIC IDPU, PLASTIC LVC/EMI Filter, and IDPU-PLASTIC Instrument Harness.

During the seven years constituting Phase A through D, many other individuals made substantial contributions to the design, development, fabrication, testing, calibration, and data definition of the PLASTIC instruments. At the University of New Hampshire: our best wishes to our colleague K. Crocker, who retired early for health reasons, and we remember the surviving family of our colleague F. Di Donna. Specific thanks to A. Ames, A. Anderson, S. Battel, E. Coutre, K. Crocker, F. Di Donna, R. DiFabio, T. Enrigo, R. Kearney, and J. Vandiver. We thank D. Curtis of the IMPACT team and S. Judy, J. Hengemihle, and W. Mocarsky for PLASTIC-related IDPU s/w development. UNH administrative support was provided by S. Asselin, L. Belaidi, J. Davis, P. Eckard, K. Giberson, S. Roy, D. Thibault, C. Williams and R. Williams. C. Kustra and K. Donahue provided meeting support; web support by J. Heirtzler. The PI particularly thanks our four frequent flyers who handled the instrument operations at APL, GSFC, Astrotech, and on the launch pad at the CapeM. Popecki, K. Singer, J. Gaidos, and S. Ellis. The University of Bern acknowledges their technical support staff. Special thanks to F. Allegrini for the extensive Entrance System/Energy Analyzer prototype calibrations and design simulations. Thanks to Contraves Space AG STEREO/PLASTIC team for construction of the Entrance System/Energy Analyzer.

The entire PLASTIC team extends thanks to M. Ketters of Canberra Semiconductor NV for extensive design discussions and construction of the PLASTIC solid-state detector wafers. Our sincere gratitude to the entire APL STEREO Spacecraft Team for their support during instrument level environmental testing and spacecraft I\&T, especially during the instrument integrations, including Ed Reynolds, M. Stevens, A. Driesman. We are grateful to the NASA STEREO Project Office at Goddard Spaceflight Center for their support, including but not limited to: N. Chrissotimos, M. Delmont, R.P. Buchanan, A. Jacques, M. Jones, S. Hynes, J. Cerullo, and A. Reyes. The STEREO mission was only possible through the unfailing support at NASA Headquarters, including R. Fisher, M. Guhathakurta, and E. Christian.

The PLASTIC investigation at UNH is funded by NASA through contract NAS5-00132. The work at the University of Bern has been supported by the Swiss National Fund and PRODEX Grant C90119. 


\section{References}

M.R. Aellig, H. Grünwaldt, P. Bochsler, S. Hefti, P. Wurz, R. Kallenbach, F.M. Ipavich, A.B. Galvin, R. Bodmer, D. Hovestadt, M. Hilchenbach et al., Solar wind minor ion charge states observed with high time resolution with SOHO/ CELIAS/ CTOF, in Correlated Phenomena at the Sun, in the Heliosphere and in Geospace. ESA SP-415, 27-31 (1997)

F. Allegrini, The PLASTIC sensor on STEREO: Design of the entrance system/energy analyzer and numerical simulations of solar wind measurements. Ph.d. thesis, University of Bern, Switzerland, 2002

M.J. Aschwanden et al., Theoretical modeling for the STEREO mission. Space Sci. Rev. (2007, this issue). doi:10.1007/s11214-006-9027-8

S.J. Bame, Solar wind minor ions-recent observations, in Solar Wind Five, NASA Conf. Publ. CP-2280, ed. by M. Neugebauer (NASA Scientific and Technical Information Branch, 1983), p. 573

M.G. Baring, K.W. Ogilvie, D.C. Ellison, R.J. Forsyth, Acceleration of solar wind ions by nearby interplanetary shocks: Comparison of Monte Carlo simulations with Ulysses observations. Astrophys. J. 476, 889-902 (1997)

D. Biesecker, D.F. Webb, O.C.St. Cyr, STEREO space weather and the space weather Beacon. Space Sci. Rev. (2007, this issue). doi:10.1007/s11214-007-9165-7

L.M. Blush, F. Allegrini, P. Bochsler, H. Daoudi, A. Galvin, R. Karrer, L. Kistler, B. Klecker, E. Moebius, A. Optiz, M. Popecki, B. Thompson, R.F. Wimmer-Schweingruber, P. Wurz, Development and calibration of major components for the STEREO/PLASTIC (plasma and suprathermal ion composition) instrument. Adv. Space Res. 36, 1544-1556 (2005)

L.F. Burlaga, E. Sittler, F. Mariani, R. Schwenn, Magnetic loop behind an interplanetary shock: Voyager, Helios and IMP 8 observations. J. Geophys. Res. 86, 6673-6684 (1981)

L.F. Burlaga, Magnetic clouds: Constant alpha force-free configurations. J. Geophys. Res. 93, 7217 (1988)

L.F. Burlaga, Large scale fluctuations, in Interplanetary Magnetohydrodynamics (Oxford University Press, New York, 1995), pp. 169-200

J.-L. Bougeret, K. Goetz, M.L. Kaiser et al., S/WAVES: The radio and plasma wave investigation on the STEREO mission. Space Sci. Rev. (2007, this issue)

H.V. Cane, T.T. von Rosenvinge, C.M.S. Cohen, R.A. Mewaldt, Two components in major solar particle events. Geophys. Res. Lett. 30(12), SEP 5-1 (2003)

K. Chotoo, N.A. Schwadron, G.M. Mason, T.H. Zurbuchen, G. Gloeckler, A. Posner, L.A. Fisk, A.B. Galvin, D.C. Hamilton, M.R. Collier, The suprathermal seed population for corotating interaction regions at 1 AU deduced from composition and spectra of $\mathrm{H}+, \mathrm{He}++$, and $\mathrm{He}+$ observed on Wind. J. Geophys. Res. 105, 23107-23122 (2000)

M.R. Collier, R.A. Lundgren, D.C. Hamilton, Channeling in ion implanted silicon solid-state detectors, University of Maryland Technical Report, PP 89-001, Dept. of Physics and Astronomy, and the Institute for Physical Science and Technology, University of Maryland, 1988

N. Crooker, J.T. Gosling, E.J. Smith, C.T. Russell, A bubblelike coronal mass ejection flux rope in the solar wind, in Physics of Magnetic Flux Ropes, ed. by C.T. Russell, E.R. Priest, L.C. Lee. Geophys. Monogr. Ser., vol. 58 (AGU, Washington, 1990), pp. 365

S.R. Cranmer et al., An empirical model of a polar coronal hole at solar minimum. Astrophys. J. 511, 481-501 (1999)

D.A. Dahl, SIMION 3D Version 6.0 User's Manual (Ion Source Software, Idaho National Engineering Laboratory, 1995)

M.H. Denton, J.E. Borovsky, R.M. Skoug, M.F. Thomsen, B. Lavraud, M.G. Henderson, R.L. McPherron, J.C. Zhang, M.W. Liemohn, Geomagnetic storms driven by ICME- and CIR-dominated solar wind. J. Geophys. Res. 111, A07S07 (2006). doi:10.1029/2005JA011436

M.I. Desai, G.M. Mason, J.R. Dwyer, J.E. Mazur, C.W. Smith, R.M. Skoug, Acceleration of 3He nuclei at interplanetary shocks. Astrophys. J. 553(1), L89-L92 (2001)

J.R. Dwyer, G.M. Mason, J.E. Mazur, J.R. Jokipii, T.T. von Rosenvinge, R.P. Lepping, Perpendicular transport of low energy corotating interaction region-associated nuclei. Astrophys. J. 490, L115-L118 (1997)

H. Ewald, H. Liebl, Der Astigmatismus des Toroidkondensators. Z. Naturforschung 10a, 872-876 (1955)

C.J. Farrugia et al., A reconnection layer associated with a magnetic cloud. Adv. Space Res. 28(5), 759 (2001)

C.J. Farrugia et al., Wind and ACE observations during the great flow of 1-4 May 1998: Relation to solar activity and implications for the magnetosphere. J. Geophys. Res. 107(A9), SSH 3-1 (2002). doi:10.1029/2001JA000188

C.J. Farrugia et al., Evolution of magnetic clouds from 0.3 AU to 1 AU: A joint Helios-Wind Investigation. Sol. Wind 11, 723-726 (2005a)

C.J. Farrugia et al., Cross-correlation of interplanetary parameters for large ( $\sim 40 \mathrm{Re})$ separation: Dependence on interplanetary structure. Sol. Wind 11, 719 (2005b) 
C.J. Farrugia etal., Interplanetary coronal mass ejection and ambient interplanetary magnetic field correlations during the Sun-Earth connection events of October-November 2003. J. Geophys. Res. 110 (2005c). doi: $10.1029 / 2004$ JA10968

T.G. Forbes et al., CME theory and models. Space Sci. Rev. 123, 251-302 (2006)

A.B. Galvin, F.M. Ipavich, G. Gloeckler, D. Hovestadt, S. Bame, B. Klecker, M. Scholer, B.T. Tsurutani, Solar wind iron charge states preceding a driver plasma. J. Geophys. Res. 92(A11), 12069-12081 (1987)

A.B. Galvin, G. Gloeckler, F.M. Ipavich, C.M. Shafer, J. Geiss, K. Ogilvie, Solar wind composition measurements by the Ulysses SWICS experiment during transient solar wind flows. Adv. Space Res. 13(6), 75-78 (1993)

A.B. Galvin, Minor ion composition in CME-related solar wind, in Coronal Mass Ejections, Geophysical Monograph 99, ed. by N. Crooker, J.A. Joselyn, J. Feynman (American Geophysical Union, 1997), pp. 253-260

A.B. Galvin, J.L. Kohl, Whole Sun Month at solar minimum: An introduction. J. Geophys. Res. 104(A5), 9673-9678 (1999). doi:10.1029/1999JA900008

A.G. Ghielmetti, H. Balsiger, R. Baenninger, P. Eberhardt, J. Geiss, D.T. Young, Calibration system for satellite and rocket-borne ion mass spectrometers in the energy range from $5 \mathrm{eV} / \mathrm{charge}$ to $100 \mathrm{keV} / \mathrm{charge}$. Rev. Sci. Instrum. 54(4), 425-436 (1983)

S.E. Gibson, Global solar wind structure from solar minimum to solar maximum: Sources and evolution. Space Sci. Rev. 97(1/4), 69 (2001)

G. Gloeckler, J. Geiss, H. Balsiger, P. Bedini, J.C. Cain, J. Fischer, L. Fisk, A.B. Galvin, F. Gliem, D.C. Hamilton, J.V. Hollweg, F.M. Ipavich, R. Joos, S. Livi, R. Lundgren, J.F. McKenzie, U. Mall, K.W. Ogilvie, F. Ottens, W. Rieck, E.O. Tums, R. von Steiger, W. Weiss, B. Wilken, The solar wind ion composition spectrometer. Astron. Astrophys. Suppl. Ser. 92, 267 (1992)

G. Gloeckler et al., The solar wind and suprathermal ion composition investigation on the WIND spacecraft. Space Sci. Rev. 71, 79-124 (1995)

G. Gloeckler, J. Geiss, Interstellar and inner source pickup ions observed by SWICS on Ulysses. Space Sci. Rev. 86, 127 (1998)

G. Gloeckler, P. Bedini, P. Bochsler, L.A. Fisk, J. Geiss, F.M. Ipavich, J. Cain, J. Fischer, R. Kallenbach, J. Miller, O. Tums, R. Wimmer, Investigation of the composition of solar and interstellar matter using solar wind and pickup ion measurements with SWICS and SWIMS on the ACE spacecraft. Space Sci. Rev. 86, 497 (1998)

G. Gloeckler et al., Unusual composition of the solar wind in the 2-3 May 1998 CME observed with SWICS on ACE. Geophys. Res. Lett. 26(2), 157 (1999)

G. Gloeckler, L.A. Fisk, L.J. Lanzerotti, Acceleration of solar wind and pickup ions by shocks, in Proceedings of the Solar Wind 11 / SOHO 16, "Connecting Sun and Heliosphere" Conference (ESA SP-592), published on CDROM, 17, 2005a

G. Gloeckler, L.A. Fisk, L.J. Lanzerotti, Pickup ions upstream and downstream of shocks. AIP Conf. Proc. 781, 252-260 (2005b). doi:10.1063/1.2032705

H. Goldstein, On the field configuration in magnetic clouds, in Solar Wind Five. NASA Conf. Publ. 2280, 1983 , p. 731

M. Gonin, R. Kallenbach, P. Bochsler, A. Buergi, Charge exchange of low energy particles passing through thin carbon foils: Dependence on foil thickness and charge state yields of $\mathrm{Mg}, \mathrm{Ca}, \mathrm{Ti}, \mathrm{Cr}$ and $\mathrm{Ni}$. Nucl. Instr. Methods B 101, 313-320 (1995)

M. Hellsing, L. Karlsson, H.-O. Andren, H. Norden, Performance of a microchannel plate ion detector in the energy range 3-25 keV. J. Phys. E.: Sci. Instrum. 18, 920-925 (1985)

D. Hovestadt, M. Hilchenbach, A. Buergi, B. Klecker, P. Laeverenz, M. Scholer, H. Gruenwaldt, W.I. Axford, S. Livi, E. Marsch, B. Wilken, H.P. Winterhoff, F.M. Ipavich, P. Bedini, M.A. Coplan, A.B. Galvin, G. Gloeckler, P. Bochsler, H. Balsiger, J. Fischer, J. Geiss, R. Kallenbach, P. Wurz, K.-U. Reiche, F. Gliem, D.L. Judge, H.S. Ogawa, K.C. Hsieh, E. Moebius, M.A. Lee, G.G. Managadze, M.I. Verigin, M. Neugebauer, CELIAS: The charge, element, and isotope analysis system for SOHO. Sol. Phys. 162, 441 (1995)

R. Howard et al., Sun Earth Connection Coronal and Heliospheric Investigation (SECCHI) (2007, this issue)

Q. Hu, B.U.O. Sonnerup, Reconstruction of magnetic clouds in the solar wind: Orientation and configuration. J. Geophys. Res. 107 (2002). doi:10.1029/2001JA000293

A.J. Hundhausen, The origin and propagation of coronal mass ejections, in Proc. 6th Intl. Solar Wind Conf., ed. by V.J. Pizzo, T.E. Holzer, D.G. Sime (NCAR Technical Note NCAR/TN-306+Proc.), 1988, 1, 131

L. Janoo, C.J. Farrugia, R.B. Torbert, J.M. Quinn et al., Field and flow perturbations in the October 18-19, 1995, magnetic cloud. J. Geophys. Res. 103, 17249 (1998)

S.W. Kahler, N.R. Sheeley Jr., R.A. Howard, D.J. Michels, M.J. Koomen, R.E. McGuire, T.T. von Rosenvinge, D.V. Reames, Associations between coronal mass ejections and solar energetic proton events. J. Geophys. Res. 89, 9683-9693 (1984) 
M. Kaiser, T.A. Kucera, J.M. Davila, O.C. St. Cyr, The STEREO mission: An overview. Space Sci. Rev. (2007, this issue). doi:10.1007/s11214-007-9277-0

R. Karrer, Ion-Optical Calibration of STEREO/PLASTIC, Ph.D. Dissertation, University of Bern, 2007

J. Kawata, K. Ohya, I. Mori, A Monte Carlo simulation of ion-induced kinetic electron emission with a stochastic excitation of electrons in solids. Jpn. J. Appl. Phys. 30, 3510-3515 (1991), doi:10.1143/JJAP.30.3510

B. Klecker et al., Energetic particle observations. Space Sci. Rev. 123, 217-250 (2006). doi:10.1007/s11214006-9018-9

K.-L. Klein, G. Trottet, The origin of solar energetic particle events: Coronal acceleration versus shock wave acceleration. Space Sci. Rev. 95, 215-225 (2001)

Y.-K. Ko, L.A. Fisk, G. Gloeckler, J. Geiss, Limitations on suprathermal tails of electrons in the lower solar corona. Geophys. Res. Lett. 23, 2785-2788 (1996)

Y.-K. Ko, J. Geiss, G. Gloeckler, On the differential ion velocity in the inner solar corona and the observed solar wind ionic charge states. J. Geophys. Res. 103(A7), 14539-14546 (1998). doi:10.1029/98JA00763

Y.-K. Ko, J.C. Raymond, T.H. Zurbuchen, P. Riley, J.M. Raines, L. Strachan, Abundance variation at the vicinity of an active region and the coronal origin of the slow solar wind. Astrophys. J. 646, 1275-1287 (2006)

N. Koshida, M. Hosobuchi, Energy distribution of output electrons from a microchannel plate. Rev. Sci. Instrum. 56(7), 1329-1331 (1985)

H. Kucharek, E. Möbius, W. Li, C.F. Farrugia, M.A. Popecki, A.B. Galvin, B. Klecker, M. Hilchenbach, P. Bochsler, On the source and acceleration of energetic He+: A long-term observation with ACE/SEPICA. J. Geophys. Res. 108(A10), 8040 (2003). doi:10.1029/2003JA009938

M. Lampton, C.W. Carlson, Low-distortion resistive anodes for two-dimensional position-sensitive MCP systems. Rev. Sci. Instrum. 50(9), 1093-1097 (1979)

M. Leitner, C.J. Farrugia, C. Möstl, K.W. Ogilvie, A.B. Galvin, R. Schwenn, H.K. Biernat, Consequences of the force-free model of magnetic clouds for their heliospheric evolution. J. Geophys. Res. 112, A06113 (2007). doi:10.1029/2006JA011940

S.T. Lepri et al., Iron charge distribution as an identifier of interplanetary coronal mass ejections. J. Geophys. Res. 106(A12), 29231-29238 (2001)

S.T. Lepri, T. Zurbuchen, Iron charge state distributions as an indicator of hot ICMEs: Possible sources and temporal and spatial variation during solar maximum. J. Geophys. Res. 109(A1), A01112 (2004)

B.C. Low, Coronal mass ejections and magnetic helicity, in Proc. of the Third SOHO Workshop-Solar Dynamic Phenomena and Solar Wind Consequences. ESA SP-373, 1994, p. 123

B.S. Lüthi, Angular scattering of ions in thin carbon foils, simulations, measurements, and predictions for the PLASTIC instrument on STEREO, Diplomarbeit, University of Bern, 2003

J.G. Luhmann et al., STEREO IMPACT investigation goals, measurements, and observations overview. Space Sci. Rev. (2007, this issue). doi:10.11007/s11214-007-9170-x

S. Lundquist, Magneto-hydrostatic fields. Arkiv fuor fysik 2, 361-365 (1950)

K. Marubashi, Structure of interplanetary magnetic clouds and their solar origins. Adv. Space Res. 6(6) 335338 (1986). doi:10.1016/0273-1177(86)90172-9

G. Mason, J.E. Mazur, J.R. Dwyer, New spectral and abundance features of interplanetary heavy ions in corotating interaction regions. Astrophys. J. 486, L149-L152 (1997)

G.M. Mason et al., Origin, injection and acceleration of CIR particles: observations. Space Sci. Rev. 89, 327-367 (1999a)

G.M. Mason, J.E. Mazur, J.R. Dwyer, 3He enhancements in large solar energetic particle events. Astrophys. J. 525(2), L133-L136 (1999b)

J.R. Mazur, G.M. Mason, R.A. Mewaldt, Charge states of energetic particles from corotating interaction regions as constraints on their source. Astrophys. J. 566, 555-561 (2002). doi:10.1086/337989

P. Mazzotta, G. Mazzitelli, S. Colafranscesco, N. Vittorio, Ionization balance for optically thin plasmas: rate coefficients for all atoms and ions of the elements H to NI. Astron. Astrophys. Suppl. Ser. 133, 403-409 (1998)

W. Meckbach, G. Braunstein, N. Arista, Secondary-electron emission in the backward and forward directions from thin carbon foils traversed by 25-250 keV proton beams. J. Phys. B: At. Mol. Phys. 8, L344-L349 (1975)

E. Möbius, D. Hovestadt, B. Klecker, M. Scholer, G. Gloeckler, F.M. Ipavich, Direct observation of He+ pick-up ions of interstellar origin in the solar wind. Nature 318, 426 (1985)

E. Möbius, D. Rucinski, M.A. Lee, P. Isenberg, Decreases in the antisunward flux of interstellar pickup He+ associated with radial interplanetary magnetic field. J. Geophys. Res. 103, 257 (1998)

E. Möbius, M. Popecki, B. Klecker, L.M. Kistler, A. Bogdanov, A.B. Galvin et al., Energy dependence of the ionic charge state distribution during the November 1997 solar energetic particle event. Geophys. Res. Lett. 26, 145-148 (1999) 
E. Möbius, D. Morris, M.A. Popecki, B. Klecker, L.M. Kistler, A.B. Galvin, Charge states of energetic $(\sim 0.5$ $\mathrm{MeV} / \mathrm{n})$ ions in corotating interaction regions at $1 \mathrm{AU}$ and implications on source populations. Geophys. Res. Lett. 29(2), (2002). doi:10.1029/2001GL013410

M. Neugebauer, R. Goldstein, Particle and field signatures of coronal mass ejections in the solar wind, in Coronal Mass Ejections, Geophysical Monograph 99, ed. by N. Crooker, J.A. Joselyn, J. Feynman (American Geophysical Union, 1997), pp. 245-251

R.O. Neukomm, Composition of coronal mass ejections derived with SWICS/Ulysses, Ph.D. dissertation, University of Bern, 1998

M. Oetliker, Response of a passivated implanted planar silicon (PIPS) detector for heavy ions with energies between 25 and $360 \mathrm{keV}$. Nucl. Instrum. Methods Phys. Res. A 337, 145-148 (1993)

G. Poletto, S.T. Suess, D.A. Biesecker, R. Esser, G. Gloeckler, Y.-K. Ko, T.H. Zurbuchen, Low-latitude solar wind during the fall 1998 SOHO-Ulysses quadrature. J. Geophys. Res. 107(A10), 1300 (2001). doi:10.1029/2001JA000275

M.A. Popecki, T.H. Zurbuchen, R.M. Skoug, C.W. Smith, A.B. Galvin, M. Lee, E. Möbius, A.T. Bogdanov, G. Gloeckler, S. Hefti, L.M. Kistler, B. Klecker, N.A. Schwadron, Simultaneous high Fe charge state measurements by solar energetic particle and solar wind instruments, AIP Conference Proceedings, September 15, 2000, vol. 528, Acceleration and Transport of Energetic Particles Observed in the Heliosphere: ACE 2000 Symposium, 2000, pp. 139-142

M.A. Popecki, Observations of energy-dependent charge states of solar energetic particles. Geophys. Monogr. Ser. 165, 127-135 (2006)

D.V. Reames, Particle acceleration at the sun and in the heliosphere. Space Sci. Rev. 90, 413-491 (1999)

P. Riley et al., Evidence of post-eruption reconnection associated with coronal mass ejections in the solar wind. Astrophys. J. 578 (2002)

P. Riley, N.U. Crooker, Kinematic treatment of coronal mass ejection evolution in the solar wind. Astrophys. J. 600, 1035-1042 (2004)

P. Riley, Modeling corotating interaction regions: from the Sun to 1 AU. J. Atmospheric Sol.-Terr. Phys. 69, 32-42 (2007). doi:10.1016/j.jastp.2006.06.008

I.G. Richardson, H.V. Cane, Identification of interplanetary coronal mass ejections at 1 AU using multiple solar wind plasma composition anomalies. J. Geophys. Res. 109, A09104 (2004a). doi:10.1029/2004JA010598

I.G. Richardson, H.V. Cane, The fraction of interplanetary coronal mass ejections that are magnetic clouds: Evidence for a solar cycle variation. Geophys. Res. Lett. 31, L18804 (2004b). doi10.1029/ 2004GL020958

S.M. Ritzau, R.A. Baragiola, Electron emission from carbon foils induced by keV ions. Phys. Rev. B 58, 2529-2538 (1998)

L. Saul, E. Möbius, P. Isenberg, P. Bochsler, On pitch-angle scattering rates of interstellar pickup ions as determined by in situ measurement of velocity distributions. Astrophys. J. 655, 672-677 (2007)

M. Steinacher, F. Jost, U. Schwab, A modern and fully automated calibration system for space ion mass spectrometers. Rev. Sci. Instrum. 66(8), 4180-4187 (1995)

E. Steinbauer, P. Bauer, M. Geretschlaeger, G. Bortels, J.P. Biersack, P. Burger, Energy resolution of silicon detectors - approaching the physical limit. Nucl. Instrum. Methods in Phys. Res. B 85, 642-649 (1994)

G.E. Thomas, The interstellar wind and its influence on the interplanetary environment. Ann. Rev. Earth Planet. Sci. 6, 173 (1978)

A.J. Tylka et al., Shock geometry, seed populations, and the origin of variable elemental composition at high energies in large gradual solar particle events. Astrophys. J. 625, 474-495 (2005)

B.J. Vasquez, C.J. Farrugia et al., The nature of fluctuations on directional discontinuities inside solar ejecta: Wind observations and theoretical interpretation. J. Geophys. Res. 106, 29283 (2001)

T.T. von Rosenvinge, H.V. Cane, Solar energetic particles: An overview, in Solar Eruptions and Energetic Particles, ed. by N. Gopalswamy, R.A. Mewaldt, J. Torsti. AGU Monogr. Ser. 165, 103-114 (2006)

G. Wehner, [De]Energieverteilung der von 2, 5, 10 und $15 \mathrm{keV} \mathrm{He-} \mathrm{und} \mathrm{Ar-ionen} \mathrm{an} \mathrm{Molybdaen} \mathrm{asugeloesten}$ electronen. Z. Physik A Hadrons Nucl., 439-442 (1966). doi:10.1007/BF01326441

R.F. Wimmer-Schweingruber, R. von Steiger, R. Paerli, Solar wind stream interfaces in corotating interaction regions: SWICS/Ulysses results. Geophys. Res. 102, 17407-17417 (1997)

R.F. Wimmer-Schweingruber, R. v. Steiger, J. Geiss, G. Gloeckler, F.M. Ipavich, B. Wilken, $\mathrm{O}^{5+}$ in the high-speed streams: SWICS/Ulysses results. Space Sci. Rev. 85, 387-396 (1998)

R.F. Wimmer-Schweingruber, R. v. Steiger, R. Paerli, Solar wind stream interfaces in corotating interaction regions: New SWICS/Ulysses results. Geophys. Res. 104, 9933-9945 (1999)

R.F. Wimmer-Schweingruber, The composition of the solar wind. Adv. Space Res. 3(0), $23-32$ (2002)

R.F. Wimmer-Schweingruber, Coronal mass ejections, a personal workshop summary. Space Sci. Rev. 123, 471-480 (2006) 
R.F. Wimmer-Schweingruber et al., Understanding interplanetary coronal mass ejection signatures. Space Sci. Rev. 123, 177-216 (2006)

H. Wollnik, T. Matsuo, H. Matsuda, The electrostatic potential in a toroidal condenser. Nucl. Instrum. Methods 102, 13-17 (1972)

D.T. Young, S.J. Bame, M.F. Thomsen, R.H. Martin, J.L. Burch, J.A. Marshall, B. Reinhard, $2 \pi$-radian fieldof-view toroidal electrostatic analyzer. Rev. Sci. Instrum. 59(5), 743-751 (1988)

D.T. Young, J.A. Marshall, J.L. Burch, S.J. Bame, R.H. Martin, A $360^{\circ}$ field-of-view toroidal ion composition analyzer using time-of-flight, in Yosemite Conference on Outstanding Problems in Solar System Plasma Physics: Theory and Instrumentation, 1989, pp. 171-176

T.H. Zurbuchen, I.G. Richardson, In-situ solar wind and magnetic field signatures of interplanetary coronal mass ejections. Space Sci. Rev. 123, 31-43 (2006) 